\title{
A New Stereoselective Synthesis of Ciguatoxin Right Wing Fragments
}

Masayuki Inoue, ${ }^{*}$ Shuji Yamashita, Atsushi Tatami, Keisuke Miyazaki and Masahiro Hirama*

Department of Chemistry, Graduate School of Science, Tohoku University, and

SORST, Japan Science and Technology Agency (JST), Sendai 980-8578, Japan

\section{Supporting Information}

86 Pages

Contents:

S1-S47: $\quad$ Experimental

S48-S86: $\quad 500 \mathrm{MHz}^{1} \mathrm{H}$ NMR spectra 


\section{Experimental}

All reactions sensitive to air or moisture were carried out under argon or nitrogen atmosphere in dry, freshly distilled solvents under anhydrous conditions, unless otherwise noted. THF was distilled from sodium/benzophenone, diethyl ether $\left(\mathrm{Et}_{2} \mathrm{O}\right)$ from $\mathrm{LiAlH}_{4}$, dichloromethane $\left(\mathrm{CH}_{2} \mathrm{Cl}_{2}\right)$, pyridine, triethylamine $\left(\mathrm{Et}_{3} \mathrm{~N}\right)$ and toluene from calcium hydride, and DMF, DMSO and HMPA from calcium hydride under reduced pressure. All other reagents were used as supplied unless otherwise stated.

Analytical thin-layer chromatography (TLC) was performed using E. Merck Silica gel 60 F254 precoated plates. Column chromatography was performed using 100-210 $\mu \mathrm{m}$ Silica Gel 60N (Kanto Chemical Co., Inc.), and for flash column chromatography 40-50 $\mu \mathrm{m}$ Silica Gel 60N (Kanto Chemical Co., Inc.) was used. Chemical shifts are reported in $\delta(\mathrm{ppm})$ using residual $\mathrm{CHCl}_{3}$ as an internal standard of $\delta 7.26$ and $\delta 77.00$ for ${ }^{1} \mathrm{H}-$ and ${ }^{13} \mathrm{C}-\mathrm{NMR}$, respectively. Signal patterns are indicated as s, singlet; d, doublet; t, triplet; q, quartet; m, multiplet; br, broad peak.

NAP ether 20. A solution of $(\mathrm{COCl})_{2}(2.4 \mathrm{~mL}, 27 \mathrm{mmol})$ in $\mathrm{CH}_{2} \mathrm{Cl}_{2}$ was cooled to $-80{ }^{\circ} \mathrm{C}$ and treated with DMSO (2.6 mL, $36 \mathrm{mmol})$. To this solution was added alcohol $17(4.68 \mathrm{~g}, 18.0 \mathrm{mmol})$ in $\mathrm{CH}_{2} \mathrm{Cl}_{2}$ (40 mL). After being stirred at $-80{ }^{\circ} \mathrm{C}$ for $15 \mathrm{~min}$, the reaction mixture was treated with $\mathrm{Et}_{3} \mathrm{~N}(10.0 \mathrm{~mL}$, $72 \mathrm{mmol}$ ), allowed to warm to $-60{ }^{\circ} \mathrm{C}$ over $2 \mathrm{~h}$, and then quenched with saturated aqueous $\mathrm{NH}_{4} \mathrm{Cl}$. The mixture was extracted with hexane/EtOAc (x2). The organic layer was washed with saturated aqueous $\mathrm{NH}_{4} \mathrm{Cl}$ and brine, and dried over $\mathrm{MgSO}_{4}$. Concentration and florisil column chromatography (hexane/EtOAc 10:1) gave aldehyde 18, which was used in the next reaction without further purification. 
To a solution of aldehyde 18 in toluene $(90 \mathrm{~mL})$ at $-80{ }^{\circ} \mathrm{C}$ was added $(R, R)$-diisopropyl tartrate $(\mathrm{Z})$ crotylboronate $(\mathbf{1 9}, 0.7 \mathrm{M}$ in toluene, $27 \mathrm{~mL}, 19 \mathrm{mmol})$ dropwise over $20 \mathrm{~min}$. After $15 \mathrm{~min}$ at $-70{ }^{\circ} \mathrm{C}$, $\mathrm{NaBH}_{4}(150 \mathrm{mg})$ and $\mathrm{EtOH}(25 \mathrm{~mL})$ were added to reduce the unreacted $\mathbf{1 8}$, and the mixture was allowed to warm to room temperature. The solution was treated with $15 \%$ aqueous $\mathrm{NaOH}(10 \mathrm{~mL})$, stirred for $3 \mathrm{~d}$, and then extracted with $\mathrm{Et}_{2} \mathrm{O}(\mathrm{x} 2)$. The organic layer was washed with brine, and dried over $\mathrm{MgSO}_{4}$. Concentration and flash column chromatography (hexane/EtOAc 15:1-10:1) gave the homoallyl alcohol (4.8 g), which was subjected to the next reaction without further purification.

To a solution of the homoallyl alcohol $(4.8 \mathrm{~g})$ in THF/DMF $(1: 1,10 \mathrm{~mL})$ at $0{ }^{\circ} \mathrm{C}$ were sequentially added $\mathrm{NaH}(1.3 \mathrm{~g}, 60 \%$ oil suspension, $32 \mathrm{mmol})$ and $\mathrm{NAPBr}(5.1 \mathrm{~g}, 23 \mathrm{mmol})$. After being stirred at room temperature for $7 \mathrm{~h}$, the mixture was treated with $\mathrm{MeOH}$, and extracted with hexane/EtOAc (x2). The organic layer was washed with saturated aqueous $\mathrm{NH}_{4} \mathrm{Cl}$ and brine, and dried over $\mathrm{MgSO}_{4}$. Concentration and flash column chromatography (hexane/EtOAc 1:0-20:1) gave NAP ether 20 (4.26 g, $9.36 \mathrm{mmol}$ ) in 52\% from 17: pale yellow oil; $[\alpha]_{\mathrm{D}}{ }^{27}=-37.1\left(\mathrm{c} 1.10, \mathrm{CHCl}_{3}\right)$; IR (film) $\vee 2975,1601$, 1461, 1095, 1032, $918 \mathrm{~cm}^{-1} ;{ }^{1} \mathrm{H}$ NMR $\left(500 \mathrm{MHz} \mathrm{CDCl}_{3}\right) \delta 0.91(3 \mathrm{H}, \mathrm{d}, J=7.0 \mathrm{~Hz}, \mathrm{Me} 57), 1.05(3 \mathrm{H}, \mathrm{d}$, $J=6.0 \mathrm{~Hz}, \mathrm{Me} 56), 1.11(3 \mathrm{H}, \mathrm{d}, J=7.0 \mathrm{~Hz}, \mathrm{Me} 55), 1.53(1 \mathrm{H}, \mathrm{dq}, J=11.5,7.0 \mathrm{~Hz}, \mathrm{H} 48), 1.67$ (1H, ddq, $J=11.5,10.0,6.0 \mathrm{~Hz}, \mathrm{H} 47), 1.78(1 \mathrm{H}, \mathrm{m}, \mathrm{H} 51), 1.81(1 \mathrm{H}, \mathrm{dd}, J=12.0,5,0 \mathrm{~Hz}, \mathrm{H} 50), 1.93$ (1H, ddd, $J$ $=12.0,6.5,3.0 \mathrm{~Hz}, \mathrm{H} 50), 2.01(1 \mathrm{H}, \mathrm{m}, \mathrm{H} 51), 2.62(1 \mathrm{H}, \mathrm{ddq}, J=7.0,7.0,7.0 \mathrm{~Hz}, \mathrm{H} 43), 3.28(1 \mathrm{H}, \mathrm{dd}, J$ $=10.0,10.0 \mathrm{~Hz}, \mathrm{H} 46), 3.36(3 \mathrm{H}, \mathrm{s}, \mathrm{MOM}), 3.37(1 \mathrm{H}, \mathrm{d}, J=7.0 \mathrm{~Hz}, \mathrm{H} 44), 3.82(1 \mathrm{H}, \mathrm{ddd}, J=8.0,8.0$, $8.0 \mathrm{~Hz}, \mathrm{H} 52), 3.89$ (1H, ddd, $J=8.0,8.0,5.0 \mathrm{~Hz}, \mathrm{H} 52), 4.05(1 \mathrm{H}, \mathrm{d}, J=10.0 \mathrm{~Hz}, \mathrm{H} 45), 4.64(1 \mathrm{H}, \mathrm{d}, J=$ $6.5 \mathrm{~Hz}, \mathrm{MOM}), 4.67(1 \mathrm{H}, \mathrm{d}, J=12.0 \mathrm{~Hz}, \mathrm{NAP}), 4.81(1 \mathrm{H}, \mathrm{d}, J=6.5 \mathrm{~Hz}, \mathrm{MOM}), 4.91(1 \mathrm{H}, \mathrm{d}, J=12.0$ Hz, NAP), 4.99 (1H, dd, $J=10.5,1.5 \mathrm{~Hz}, \mathrm{H} 41), 5.04(1 \mathrm{H}$, brd, $J=17.5 \mathrm{~Hz}, \mathrm{H} 41), 5.78(1 \mathrm{H}, \mathrm{ddd}, J=$ 
17.5, 10.5, 7.0 Hz, H42), 7.43-7.50 (3H, m, NAP), 7.76-7.88 (4H, m, NAP); ${ }^{13} \mathrm{C}$ NMR (125 MHz, $\left.\mathrm{CDCl}_{3}\right) \delta 13.6,15.8,17.6,24.3,35.0,40.0,40.2,42.1,56.3,67.3,72.9,73.0,80.7,83.5,98.4,107.9$ 114.6, 125.6, 125.9, 126.2, 126.4, 127.7, 127.80, 127.84, 132.8, 133.2, 136.6, 142.3; HRMS (MALDI), calcd for $\mathrm{C}_{28} \mathrm{H}_{38} \mathrm{O}_{5} \mathrm{Na} 477.2617\left(\mathrm{M}+\mathrm{Na}^{+}\right)$, found 477.2635.

Carboxylic acid 10. To a solution of NAP ether 20 (271 mg, $0.60 \mathrm{mmol})$ and NMO (50\% aqueous solution, $0.36 \mathrm{~mL}, 1.8 \mathrm{mmol})$ in $t-\mathrm{BuOH} / \mathrm{H}_{2} \mathrm{O}(1: 1,1.6 \mathrm{~mL})$ at room temperature was added $\mathrm{OsO}_{4}(38$ $\mathrm{mM}$ in $t$ - $\mathrm{BuOH}, 160 \mu \mathrm{l}, 6.0 \mu \mathrm{mol})$. After being stirred for $2 \mathrm{~d}$, the solution was treated with $\mathrm{NaIO}_{4}$ (385 mg, $1.8 \mathrm{mmol}$ ), stirred for additional $1 \mathrm{~h}$, and then diluted with aqueous $\mathrm{NaHCO}_{3}$. The mixture was extracted with $\mathrm{Et}_{2} \mathrm{O}(\mathrm{x} 3)$, and the organic layer was washed with brine, dried over $\mathrm{MgSO}_{4}$, and concentrated to give the aldehyde, which was used in the next reaction without further purification.

To a solution of the aldehyde in $t-\mathrm{BuOH} / \mathrm{H}_{2} \mathrm{O}(4: 1,2 \mathrm{~mL})$ at room temperature were added $\mathrm{NaH}_{2} \mathrm{PO}_{4} \cdot 2 \mathrm{H}_{2} \mathrm{O}(189 \mathrm{mg}, 1.2 \mathrm{mmol})$, 2-methyl-2-butene $(1 \mathrm{~mL}, 8.0 \mathrm{mmol})$, and $\mathrm{NaClO}_{2}(257 \mathrm{mg}, 2.8$ mmol). After being stirred at room temperature for $3 \mathrm{~h}$, the mixture was extracted with EtOAc (x2), and the organic layer was washed with brine and dried over $\mathrm{MgSO}_{4}$. Concentration and flash column chromatography (hexane/EtOAc 10:1-1:1) gave carboxylic acid $\mathbf{1 0}$ (280 mg, $0.59 \mathrm{mmol})$ in $99 \%$ yield over 2 steps: pale yellow oil; $[\alpha]_{\mathrm{D}}{ }^{19}=-45.5\left(c 1.00, \mathrm{CHCl}_{3}\right)$; IR (film) $v 3056,2975,1705,1460,1030$, $754 \mathrm{~cm}^{-1} ;{ }^{1} \mathrm{H}$ NMR $\left(500 \mathrm{MHz}, \mathrm{CDCl}_{3}\right) \delta 0.91(3 \mathrm{H}, \mathrm{d}, J=7.0 \mathrm{~Hz}, \mathrm{Me} 57), 1.03(3 \mathrm{H}, \mathrm{d}, J=6.5 \mathrm{~Hz}, \mathrm{Me} 56)$, $1.33(3 \mathrm{H}, \mathrm{d}, J=6.5 \mathrm{~Hz}, \mathrm{Me} 55), 1.54(1 \mathrm{H}, \mathrm{dq}, J=11.5,7.0 \mathrm{~Hz}, \mathrm{H} 48), 1.71(1 \mathrm{H}, \mathrm{ddq}, J=11.5,10.0,6.5$ Hz, H47), 1.75 (1H, m, H51), 1.81 (1H, ddd, $J=12.5,8.5,5.0 \mathrm{~Hz}, \mathrm{H} 50), 1.91(1 \mathrm{H}, \mathrm{ddd}, J=12.5,10.0$ $7.0 \mathrm{~Hz}, \mathrm{H} 50), 2.06$ (1H, m, H51), $2.91(1 \mathrm{H}, \mathrm{dq}, J=6.5,6.5 \mathrm{~Hz}, \mathrm{H} 43), 3.12(1 \mathrm{H}, \mathrm{dd}, J=10.0,10.0 \mathrm{~Hz}$ 
H46), 3.32 (3H, s, MOM), 3.83-3.92 (2H, m, H52x2), 4.04 (1H, d, J = $10.0 \mathrm{~Hz}, \mathrm{H} 45), 4.07$ (1H, d, J = $6.5 \mathrm{~Hz}, \mathrm{H} 44), 4.64(1 \mathrm{H}, \mathrm{d}, J=6.5 \mathrm{~Hz}, \mathrm{MOM}), 4.74(1 \mathrm{H}, \mathrm{d}, J=12.0 \mathrm{~Hz}, \mathrm{NAP}), 4.75(1 \mathrm{H}, \mathrm{d}, J=6.5 \mathrm{~Hz}$, MOM), $4.91(1 \mathrm{H}, \mathrm{d}, J=12.0 \mathrm{~Hz}, \mathrm{NAP}), 7.45-7.52(3 \mathrm{H}, \mathrm{m}, \mathrm{NAP}), 7.78-7.84(4 \mathrm{H}, \mathrm{m}, \mathrm{NAP}) ;{ }^{13} \mathrm{C}$ NMR $\left(125 \mathrm{MHz}, \mathrm{CDCl}_{3}\right) \delta 13.5,13.7,15.7,24.1,34.6,40.1,41.2,41.7,56.2,67.6,72.3,73.5,79.2,81.4,98.5$, 108.0, 125.82, 125.84, 126.1, 126.5, 127.6, 127.8, 128.1, 132.9, 133.2, 135.5, 179.0; HRMS (FAB), calcd for $\mathrm{C}_{27} \mathrm{H}_{36} \mathrm{O}_{7} \mathrm{Na} 495.2359\left(\mathrm{M}+\mathrm{Na}^{+}\right)$, found 495.2365.

Methylacetal 21. To a solution of hemiacetal $15(59.4 \mathrm{~g}, 169.3 \mathrm{mmol})$ and $\mathrm{CH}(\mathrm{OMe})_{3}(93 \mathrm{~mL}, 850$ mmol) in benzene $(340 \mathrm{~mL})$ at room temperature was added CSA (0.8 g, $3.4 \mathrm{mmol})$. After being stirred at room temperature for $6 \mathrm{~h}$, the reaction mixture was quenched with $\mathrm{Et}_{3} \mathrm{~N}(20 \mathrm{~mL})$. Concentration and flash column chromatography (hexane/EtOAc 20:1-5:1) gave methylacetal 21 (63.9 $\mathrm{g}, 167 \mathrm{mmol})$ in $99 \%$ yield: colorless oil; $[\alpha]_{\mathrm{D}}^{30}=-94.7\left(c 1.09, \mathrm{CHCl}_{3}\right)$; IR (neat) $v 2977,1454,1148$, 1099, 1021, 920, $698 \mathrm{~cm}^{-1} ;{ }^{1} \mathrm{H}$ NMR $\left(500 \mathrm{MHz}, \mathrm{CDCl}_{3}\right) \delta 0.88(3 \mathrm{H}, \mathrm{d}, J=6.5 \mathrm{~Hz}, \mathrm{Me} 57), 0.98(3 \mathrm{H}, \mathrm{d}, J$ $=6.5 \mathrm{~Hz}, \mathrm{Me} 56), 1.45(1 \mathrm{H}, \mathrm{dq}, J=10.0,6.5 \mathrm{~Hz}, \mathrm{H} 48), 1.72(1 \mathrm{H}, \mathrm{ddq}, J=10.0,10.0,6.5 \mathrm{~Hz}, \mathrm{H} 47), 2.27$ $(1 \mathrm{H}, \mathrm{dd}, J=14.0,9.0 \mathrm{~Hz}, \mathrm{H} 50), 2.63(1 \mathrm{H}, \mathrm{ddd}, J=14.0,5.0,1.5 \mathrm{~Hz}, \mathrm{H} 50), 3.07(1 \mathrm{H}, \mathrm{dd}, J=10.0,10.0$ Hz, H46), 3.21 (3H, s, MeO), 3.35 (3H, s, MOM), $3.57(1 \mathrm{H}, \mathrm{dt}, J=10.0,3.5 \mathrm{~Hz}, \mathrm{H} 45), 3.68(2 \mathrm{H}, \mathrm{d}, J=$ $3.5 \mathrm{~Hz}, \mathrm{H} 44 \mathrm{x} 2), 4.59(1 \mathrm{H}, \mathrm{d}, J=6.0 \mathrm{~Hz}, \mathrm{MOM}), 4.60(1 \mathrm{H}, \mathrm{d}, J=12.0 \mathrm{~Hz}, \mathrm{Bn}), 4.64(1 \mathrm{H}, \mathrm{d}, J=12.0 \mathrm{~Hz}$, Bn), $4.69(1 \mathrm{H}, \mathrm{d}, J=6.0 \mathrm{~Hz}, \mathrm{MOM}), 5.04(1 \mathrm{H}, \mathrm{dd}, J=9.0,1.5 \mathrm{~Hz}, \mathrm{H} 52), 5.07(1 \mathrm{H}, \mathrm{d}, J=16.5 \mathrm{~Hz}, \mathrm{H} 52)$, $5.74(1 \mathrm{H}$, dddd $, J=16.5,9.0,9.0,5.0 \mathrm{~Hz}, \mathrm{H} 51), 7.26-7.36(5 \mathrm{H}, \mathrm{m}, \mathrm{Bn}) ;{ }^{13} \mathrm{C} \mathrm{NMR}\left(125 \mathrm{MHz}, \mathrm{CDCl}_{3}\right) \delta$ $12.5,15.1,37.4,38.6,41.5,47.3,56.2,69.8,72.7,73.2,79.5,98.2,100.7,117.0,127.3,127.4,128.2$, 134.2, 138.7; MALDI-TOF MS, calcd for $\mathrm{C}_{21} \mathrm{H}_{32} \mathrm{O}_{5} \mathrm{Na} 387.21\left(\mathrm{M}+\mathrm{Na}^{+}\right)$, found 387.23; Anal. calcd for 
$\mathrm{C}_{21} \mathrm{H}_{32} \mathrm{O}_{5}: \mathrm{C}, 65.55, \mathrm{H}, 8.25$, found $\mathrm{C}, 65.23, \mathrm{H}, 8.34$.

Diols 22 and 23. To a mixture of methylacetal 21 (1.94 g, $5.32 \mathrm{mmol})$, (DHQ) $)_{2}$ PYR (70 mg, 0.08 $\mathrm{mmol}), \mathrm{K}_{3} \mathrm{Fe}(\mathrm{CN})_{6}(8.75 \mathrm{~g}, 26.6 \mathrm{mmol})$ and $\mathrm{K}_{2} \mathrm{CO}_{3}(3.67 \mathrm{~g}, 26.6 \mathrm{mmol})$ in $t-\mathrm{BuOH} / \mathrm{H}_{2} \mathrm{O}(1: 1,53.2 \mathrm{~mL})$ at $0{ }^{\circ} \mathrm{C}$ was added $\mathrm{OsO}_{4}(19 \mathrm{mM}$ in $t-\mathrm{BuOH}, 2.63 \mathrm{~mL}, 0.05 \mathrm{mmol})$. Additional (DHQ) ${ }_{2} \mathrm{PYR}(105 \mathrm{mg}$, $0.12 \mathrm{mmol})$ and $\mathrm{OsO}_{4}(19 \mathrm{mM}$ in $t-\mathrm{BuOH}, 3.91 \mathrm{~mL}, 0.07 \mathrm{mmol})$ were introduced to complete the reaction. The reaction mixture was quenched with saturated aqueous $\mathrm{Na}_{2} \mathrm{~S}_{2} \mathrm{O}_{3}(20 \mathrm{~mL})$ and solid $\mathrm{Na}_{2} \mathrm{~S}_{2} \mathrm{O}_{3} \cdot 7 \mathrm{H}_{2} \mathrm{O}$, and stirred for $2 \mathrm{~h}$. The solution was extracted with $\mathrm{Et}_{2} \mathrm{O}(\mathrm{x} 2)$, and the organic layer was washed with saturated aqueous $\mathrm{Na}_{2} \mathrm{~S}_{2} \mathrm{O}_{3}(\mathrm{x} 3)$ and brine, and dried over $\mathrm{MgSO}_{4}$. Concentration and flash column chromatography $\left(\mathrm{CHCl}_{3} /\right.$ hexane 1:1) gave diol 23 (1.69 g, $\left.4.24 \mathrm{mmol}\right)$ in $79 \%$ yield and its C51-epimer $22(0.42 \mathrm{~g}, 1.05 \mathrm{mmol})$ in $20 \%$ yield. 22: colorless oil; ${ }^{1} \mathrm{H} \mathrm{NMR}\left(500 \mathrm{MHz}, \mathrm{CDCl}_{3}\right) \delta$ $0.91(3 \mathrm{H}, \mathrm{d}, J=6.5 \mathrm{~Hz}, \mathrm{Me} 57), 1.02(3 \mathrm{H}, \mathrm{d}, J=6.5 \mathrm{~Hz}, \mathrm{Me} 56), 1.63(1 \mathrm{H}, \mathrm{dd}, J=13.5,2.0 \mathrm{~Hz}, \mathrm{H} 50)$, $1.65(1 \mathrm{H}, \mathrm{dq}, J=10.5,6.5 \mathrm{~Hz}, \mathrm{H} 48), 1.78(1 \mathrm{H}, \mathrm{ddq}, J=10.5,10.5,6.5 \mathrm{~Hz}, \mathrm{H} 47), 2.03(1 \mathrm{H}, \mathrm{dd}, J=13.5$, $11.5 \mathrm{~Hz}, \mathrm{H} 50), 2.17(1 \mathrm{H}, \mathrm{t}, J=6.5 \mathrm{~Hz}, \mathrm{OH} 52), 3.20$ (3H, s, MeO), 3.25 (1H, dd, J = 10.5, $10.5 \mathrm{~Hz}, \mathrm{H} 46)$, 3.34 (3H, s, MOM), 3.45 (1H, ddd, $J=13.0,6.5,6.5 \mathrm{~Hz}, \mathrm{H} 52), 3.55$ (1H, dt, $J=10.5,2.5 \mathrm{~Hz}, \mathrm{H} 45)$, 3.58-3.66 (3H, m, H44x2, 52), 3.85-3.91 (1H, m, H51), $4.23(1 \mathrm{H}, \mathrm{s}, \mathrm{OH} 51), 4.50(1 \mathrm{H}, \mathrm{d}, J=11.5 \mathrm{~Hz}$, Bn), $4.55(1 \mathrm{H}, \mathrm{d}, J=6.5 \mathrm{~Hz}, \mathrm{MOM}), 4.57(1 \mathrm{H}, \mathrm{d}, J=11.5 \mathrm{~Hz}, \mathrm{Bn}), 4.64(1 \mathrm{H}, \mathrm{d}, J=6.5 \mathrm{~Hz}, \mathrm{MOM})$, 7.26-7.35 (5H, m, Bn); ${ }^{13} \mathrm{C}$ NMR (125 MHz, $\left.\mathrm{CDCl}_{3}\right) \delta 13.1,15.0,34.4,36.9,40.9,47.4,56.2,66.8$, $67.7,68.4,72.3,73.3,78.5,98.2,101.7,127.6,127.7,128.3,137.8 . \quad 23:$ colorless oil; $[\alpha]_{\mathrm{D}}{ }^{27}=-56.9(c$ $\left.1.09, \mathrm{CHCl}_{3}\right) ; \mathrm{IR}$ (neat) $v 3448,2937,1455,1148,1034,740,699 \mathrm{~cm}^{-1} ;{ }^{1} \mathrm{H} \mathrm{NMR}\left(500 \mathrm{MHz}, \mathrm{CDCl}_{3}\right) \delta$ $0.93(3 \mathrm{H}, \mathrm{d}, J=6.5 \mathrm{~Hz}, \mathrm{Me} 57), 0.98(3 \mathrm{H}, \mathrm{d}, J=6.5 \mathrm{~Hz}, \mathrm{Me} 56), 1.45(1 \mathrm{H}, \mathrm{dq}, J=10.5,6.5 \mathrm{~Hz}, \mathrm{H} 48)$, 
$1.74(1 \mathrm{H}, \mathrm{ddq}, J=10.5,10.5,6.5 \mathrm{~Hz}, \mathrm{H} 47), 1.85(1 \mathrm{H}, \mathrm{dd}, J=14.5,8.5 \mathrm{~Hz}, \mathrm{H} 50), 1.96(1 \mathrm{H}, \mathrm{dd}, J=14.5$, $5.5 \mathrm{~Hz}, \mathrm{H} 50), 2.37(1 \mathrm{H}, \mathrm{t}, J=6.0 \mathrm{~Hz}, \mathrm{OH} 52), 2.96(1 \mathrm{H}, \mathrm{d}, J=4.5 \mathrm{~Hz}, \mathrm{OH} 51), 3.19(1 \mathrm{H}, \mathrm{dd}, J=10.5$, $10.5 \mathrm{~Hz}, \mathrm{H} 46), 3.20$ (3H, s, MeO), 3.35 (3H, s, MOM), 3.54-3.67 (5H, m, H44x2, H45, H52x2), 3.98 $(1 \mathrm{H}, \mathrm{m}, \mathrm{H} 51), 4.53(1 \mathrm{H}, \mathrm{d}, J=12.0 \mathrm{~Hz}, \mathrm{Bn}), 4.56(1 \mathrm{H}, \mathrm{d}, J=12.0 \mathrm{~Hz}, \mathrm{Bn}), 4.57(1 \mathrm{H}, \mathrm{d}, J=6.0 \mathrm{~Hz}$, MOM), $4.65(1 \mathrm{H}, \mathrm{d}, J=6.0 \mathrm{~Hz}, \mathrm{MOM}), 7.28-7.35(5 \mathrm{H}, \mathrm{m}, \mathrm{Bn}) ;{ }^{13} \mathrm{C} \mathrm{NMR}\left(125 \mathrm{MHz}, \mathrm{CDCl}_{3}\right) \delta 12.9$, $14.9,37.3,38.0,43.7,47.6,56.1,66.6,69.0,69.1,72.1,73.2,78.8,98.1,100.2,127.5,127.7,128.2$, 137.8; HRMS (ESI), calcd for $\mathrm{C}_{21} \mathrm{H}_{34} \mathrm{O}_{7} \mathrm{Na} 421.2196\left(\mathrm{M}+\mathrm{Na}^{+}\right)$, found 421.2196.

Spiroacetals $24 \alpha$ and $24 \beta$. To a solution of diol $22(15.5 \mathrm{~g}, 38.8 \mathrm{mmol})$ in $\mathrm{CH}_{3} \mathrm{CN}(194 \mathrm{~mL})$ at $80{ }^{\circ} \mathrm{C}$ was added PPTS $(1.3 \mathrm{~g}, 3.9 \mathrm{mmol})$. After being stirred at $80{ }^{\circ} \mathrm{C}$ for $3 \mathrm{~h}$, the reaction mixture was quenched with $\mathrm{Et}_{3} \mathrm{~N}$ and saturated aqueous $\mathrm{NaHCO}_{3}$, and then extracted with EtOAc (x2). The organic layer was washed with brine, and dried over $\mathrm{Na}_{2} \mathrm{SO}_{4}$. Concentration and flash column chromatography (hexane/EtOAc 7:1-0:1) gave $\mathbf{2 4} \beta$ (10.6 g, $28.9 \mathrm{mmol})$ in 75\% yield and its C49-epimer $\mathbf{2 4 \alpha}(2.3 \mathrm{~g}, 6.2$ mmol) in $15 \%$ yield. $24 \alpha$ : colorless oil; $[\alpha]_{\mathrm{D}}{ }^{27}=10.5\left(c 1.00, \mathrm{CHCl}_{3}\right)$; IR (neat) $v 3463,2886,1454$, 1301, 1097, 966, $842 \mathrm{~cm}^{-1} ;{ }^{1} \mathrm{H}$ NMR $\left(500 \mathrm{MHz}, \mathrm{CDCl}_{3}\right) \delta 0.98(3 \mathrm{H}, \mathrm{d}, J=6.5 \mathrm{~Hz}, \mathrm{Me} 57), 1.04(3 \mathrm{H}, \mathrm{d}, J$ $=6.0 \mathrm{~Hz}, \mathrm{Me} 56), 1.32(1 \mathrm{H}, \mathrm{m}, \mathrm{H} 47), 1.67(1 \mathrm{H}, \mathrm{m}, \mathrm{H} 48), 1.82(1 \mathrm{H}, \mathrm{dd}, J=14.5,2.5 \mathrm{~Hz}, \mathrm{H} 50), 2.32(1 \mathrm{H}$, $\mathrm{dd}, J=14.5,6.5 \mathrm{~Hz}, \mathrm{H} 50), 3.12(1 \mathrm{H}, \mathrm{dd}, J=9.5,9.5 \mathrm{~Hz}, \mathrm{H} 46), 3.32$ (3H, s, MOM), 3.42 (1H, m, H45), $3.60(1 \mathrm{H}, \mathrm{dd}, J=11.0,5.5 \mathrm{~Hz}, \mathrm{H} 44), 3.69(1 \mathrm{H}, \mathrm{dd}, J=11.0,2.0 \mathrm{~Hz}, \mathrm{H} 44), 3.81(1 \mathrm{H}, \mathrm{d}, J=10.0 \mathrm{~Hz}$, H52), $4.15(1 \mathrm{H}, \mathrm{dd}, J=10.0,4.5 \mathrm{~Hz}, \mathrm{H} 52), 4.54$ (1H, m, H51), 4.55 (1H, d, $J=12.0 \mathrm{~Hz}, \mathrm{Bn}), 4.56$ (1H, $\mathrm{d}, J=7.0 \mathrm{~Hz}, \mathrm{MOM}), 4.58(1 \mathrm{H}, \mathrm{d}, J=12.0 \mathrm{~Hz}, \mathrm{Bn}), 4.65(1 \mathrm{H}, \mathrm{d}, J=7.0 \mathrm{~Hz}, \mathrm{MOM}), 7.26-7.36(5 \mathrm{H}, \mathrm{m}$, $\mathrm{Bn}) ;{ }^{13} \mathrm{C} \mathrm{NMR}\left(125 \mathrm{MHz}, \mathrm{CDCl}_{3}\right) \delta$ 13.6, 15.3, 37.8, 41.0, 42.1, 56.3, 70.1, 71.9, 73.4, 74.7, 76.1, 79.7, 
98.4, 110.5, 127.5, 127.8, 128.3, 138.4; MALDI-TOF MS, calcd for $\mathrm{C}_{20} \mathrm{H}_{30} \mathrm{O}_{6} \mathrm{Na} 389.19\left(\mathrm{M}+\mathrm{Na}^{+}\right)$, found 389.19. 24ß: colorless oil; $[\alpha]_{\mathrm{D}}{ }^{27}=-46.9\left(c 1.00, \mathrm{CHCl}_{3}\right)$; IR (neat) $v 3494,2927,1455,1365,1095$, 1033, $861 \mathrm{~cm}^{-1}$; ${ }^{1} \mathrm{H}$ NMR $\left(500 \mathrm{MHz}, \mathrm{CDCl}_{3}\right) \delta 0.90(3 \mathrm{H}, \mathrm{d}, J=6.5 \mathrm{~Hz}, \mathrm{Me} 57), 1.02(3 \mathrm{H}, \mathrm{d}, J=6.0 \mathrm{~Hz}$, Me56), $1.62(1 \mathrm{H}, \mathrm{m}, \mathrm{H} 48), 1.69(1 \mathrm{H}, \mathrm{m}, \mathrm{H} 47), 1.94(1 \mathrm{H}, \mathrm{d}, J=13.0 \mathrm{~Hz}, \mathrm{H} 50), 2.10(1 \mathrm{H}, \mathrm{dd}, J=13.0$, $5.5 \mathrm{~Hz}, \mathrm{H} 50), 3.04(1 \mathrm{H}, \mathrm{dd}, J=10.0,10.0 \mathrm{~Hz}, \mathrm{H} 46), 3.32(3 \mathrm{H}, \mathrm{s}, \mathrm{MOM}), 3.52(1 \mathrm{H}, \mathrm{dd}, J=10.5,7.0 \mathrm{~Hz}$, H44), 3.64 (1H, dd, $J=10.5,2.5 \mathrm{~Hz}, \mathrm{H} 44), 3.72(1 \mathrm{H}, \mathrm{d}, J=12.5 \mathrm{~Hz}, \mathrm{OH} 51), 3.93(1 \mathrm{H}, \mathrm{ddd}, J=10.0$, 7.0, $2.5 \mathrm{~Hz}, \mathrm{H} 45), 3.95$ (1H, d, $J=10.0 \mathrm{~Hz}, \mathrm{H} 52), 4.09$ (1H, dd, $J=10.0,4.5 \mathrm{~Hz}, \mathrm{H} 52), 4.31(1 \mathrm{H}, \mathrm{ddd}, J$ $=12.5,5.5,4.5 \mathrm{~Hz}, \mathrm{H} 51), 4.54(1 \mathrm{H}, \mathrm{d}, J=12.0 \mathrm{~Hz}, \mathrm{Bn}), 4.56(1 \mathrm{H}, \mathrm{d}, J=12.0 \mathrm{~Hz}, \mathrm{Bn}), 4.57(1 \mathrm{H}, \mathrm{d}, J=$ $7.0 \mathrm{~Hz}, \mathrm{MOM}), 4.64(1 \mathrm{H}, \mathrm{d}, J=7.0 \mathrm{~Hz}, \mathrm{MOM}), 7.25-7.35(5 \mathrm{H}, \mathrm{m}, \mathrm{Bn}) ;{ }^{13} \mathrm{C} \mathrm{NMR}\left(125 \mathrm{MHz}, \mathrm{CDCl}_{3}\right) \delta$ $13.4,15.6,39.2,41.0,42.3,56.2,69.9,71.4,71.8,73.2,77.7,80.0,98.3,108.7,127.6,127.7,128.4$, 138.0; MALDI-TOF MS, calcd for $\mathrm{C}_{20} \mathrm{H}_{30} \mathrm{O}_{6} \mathrm{Na} 389.19\left(\mathrm{M}+\mathrm{Na}^{+}\right)$, found 389.19; Anal. calcd for $\mathrm{C}_{20} \mathrm{H}_{30} \mathrm{O}_{6}: \mathrm{C}, 65.55, \mathrm{H}, 8.25$, found $\mathrm{C}, 65.25, \mathrm{H}, 8.41$.

Spiroacetals $25 \alpha$ and $25 \beta$. To a solution of diol $23(24.6 \mathrm{~g}, 61.7 \mathrm{mmol})$ in $\mathrm{CH}_{3} \mathrm{CN}(308 \mathrm{~mL})$ at $80{ }^{\circ} \mathrm{C}$ was added PPTS $(0.78 \mathrm{~g}, 3.1 \mathrm{mmol})$. After being stirred at $80{ }^{\circ} \mathrm{C}$ for $3 \mathrm{~h}$, the reaction mixture was quenched with $\mathrm{Et}_{3} \mathrm{~N}$ and saturated aqueous $\mathrm{NaHCO}_{3}$, and then extracted with $\mathrm{EtOAc}(\mathrm{x} 2)$. The organic layer was washed with brine, and dried over $\mathrm{Na}_{2} \mathrm{SO}_{4}$. Concentration and flash column chromatography (hexane/EtOAc 7:1-0:1) gave $25 \beta$ (19.2 g, $52.4 \mathrm{mmol})$ in 85\% yield and its C49-epimer $25 \alpha(2.4 \mathrm{~g}, 6.5$ mmol) in $10 \%$ yield. $\quad 25 \alpha$ : colorless oil; ${ }^{1} \mathrm{H}$ NMR $\left(500 \mathrm{MHz}, \mathrm{CDCl}_{3}\right) \delta 0.90(3 \mathrm{H}, \mathrm{d}, J=6.5 \mathrm{~Hz}, \mathrm{Me} 57)$, $1.02(3 \mathrm{H}, \mathrm{d}, J=6.5 \mathrm{~Hz}, \mathrm{Me} 56), 1.41(1 \mathrm{H}, \mathrm{dq}, J=10.0,6.5 \mathrm{~Hz}, \mathrm{H} 48), 1.71-1.77$ (2H, m, H47, H50), $2.35(1 \mathrm{H}, \mathrm{d}, J=13.5 \mathrm{~Hz}, \mathrm{H} 50), 2.99(1 \mathrm{H}, \mathrm{dd}, J=10.0,10.0 \mathrm{~Hz}, \mathrm{H} 46), 3.26(3 \mathrm{H}, \mathrm{s}, \mathrm{MOM}), 3.52$ (1H, dd, 
$J=9.5,9.0 \mathrm{~Hz}, \mathrm{H} 44), 3.67(1 \mathrm{H}, \mathrm{ddd}, J=10.0,9.0,2.0 \mathrm{~Hz}, \mathrm{H} 45), 3.75(1 \mathrm{H}, \mathrm{dd}, J=9.5,2.0 \mathrm{~Hz}, \mathrm{H} 44)$,

$3.87(1 \mathrm{H}, \mathrm{d}, J=12.5 \mathrm{~Hz}, \mathrm{OH} 51), 4.09(1 \mathrm{H}, \mathrm{d}, J=10.0 \mathrm{~Hz}, \mathrm{H} 52), 4.12(1 \mathrm{H}, \mathrm{d}, J=10.0 \mathrm{~Hz}, \mathrm{H} 52), 4.39$

$(1 \mathrm{H}, \mathrm{m}, \mathrm{H} 51), 4.51(1 \mathrm{H}, \mathrm{d}, J=12.0 \mathrm{~Hz}, \mathrm{Bn}), 4.56(1 \mathrm{H}, \mathrm{d}, J=6.5 \mathrm{~Hz}, \mathrm{MOM}), 4.58(1 \mathrm{H}, \mathrm{d}, J=6.5 \mathrm{~Hz}$,

MOM), $4.59(1 \mathrm{H}, \mathrm{d}, J=12.0 \mathrm{~Hz}, \mathrm{Bn}), 7.26-7.33(5 \mathrm{H}, \mathrm{m}, \mathrm{Bn}) ;{ }^{13} \mathrm{C} \mathrm{NMR}\left(125 \mathrm{MHz}, \mathrm{CDCl}_{3}\right) \delta 13.8,15.2$,

33.7, 41.5, 41.6, 56.2, 70.2, 71.9, 73.3, 75.9, 77.4, 80.3, 98.5, 110.7, 127.5, 127.6, 128.3, 137.8. 25

colorless oil; $[\alpha]_{\mathrm{D}}{ }^{27}=-43.2\left(c 1.44, \mathrm{CHCl}_{3}\right)$; IR (neat) $v 3463,2927,1454,1146,1027,950,738 \mathrm{~cm}^{-1}$;

${ }^{1} \mathrm{H}$ NMR (500 MHz, $\left.\mathrm{CDCl}_{3}\right) \delta 1.01(3 \mathrm{H}, \mathrm{d}, J=6.5 \mathrm{~Hz}, \mathrm{Me} 57), 1.05(3 \mathrm{H}, \mathrm{d}, J=6.5 \mathrm{~Hz}, \mathrm{Me} 56), 1.57(1 \mathrm{H}$, $\mathrm{dq}, J=11.5,6.5 \mathrm{~Hz}, \mathrm{H} 48), 1.68(1 \mathrm{H}, \mathrm{ddq}, J=11.5,10.0,6.5 \mathrm{~Hz}, \mathrm{H} 47), 1.97(1 \mathrm{H}, \mathrm{ddd}, J=14.0,1.5,1.5$ Hz, H50), 2.28 (1H, dd, $J=14.0,6.5 \mathrm{~Hz}, \mathrm{H} 50), 3.19(1 \mathrm{H}, \mathrm{dd}, J=10.0,10.0 \mathrm{~Hz}, \mathrm{H} 46), 3.40(3 \mathrm{H}, \mathrm{s}$, MOM), $3.57(1 \mathrm{H}, \mathrm{dd}, J=11.0,2.5 \mathrm{~Hz}, \mathrm{H} 44), 3.62(1 \mathrm{H}, \mathrm{dd}, J=11.0,4.0 \mathrm{~Hz}, \mathrm{H} 44), 3.73(1 \mathrm{H}, \mathrm{ddd}, J=$ 10.0, 4.0, 2.5 Hz, H45), $3.78(1 \mathrm{H}, \mathrm{d}, J=10.0 \mathrm{~Hz}, \mathrm{H} 52), 3.94(1 \mathrm{H}, \mathrm{dd}, J=10.0,4.5 \mathrm{~Hz}, \mathrm{H} 52), 4.52(1 \mathrm{H}$, m, H51), $4.53(1 \mathrm{H}, \mathrm{d}, J=12.0 \mathrm{~Hz}, \mathrm{Bn}), 4.54(1 \mathrm{H}, \mathrm{d}, J=6.0 \mathrm{~Hz}, \mathrm{MOM}), 4.59(1 \mathrm{H}, \mathrm{d}, J=12.0 \mathrm{~Hz}, \mathrm{Bn})$, $4.67(1 \mathrm{H}, \mathrm{d}, J=6.0 \mathrm{~Hz}, \mathrm{MOM}), 7.27-7.34(5 \mathrm{H}, \mathrm{m}, \mathrm{Bn}) ;{ }^{13} \mathrm{C} \mathrm{NMR}\left(125 \mathrm{MHz}, \mathrm{CDCl}_{3}\right) \delta 13.6,15.7,39.2$, $41.7,45.6,56.1,69.5,71.8,72.4,73.2,74.4,79.2,98.1,108.6,127.5,127.8,128.2,138.2$; MALDI-TOF MS, calcd for $\mathrm{C}_{20} \mathrm{H}_{30} \mathrm{O}_{6} \mathrm{Na} 389.19\left(\mathrm{M}+\mathrm{Na}^{+}\right)$, found 389.19; Anal. calcd for $\mathrm{C}_{20} \mathrm{H}_{30} \mathrm{O}_{6}: \mathrm{C}, 65.55 ; \mathrm{H}, 8.25$, found C, 65.23; H, 8.34.

Isomerization of $\mathbf{2 5} \boldsymbol{\alpha}$. To a solution of spiroacetal $\mathbf{2 5} \boldsymbol{\alpha}(52 \mathrm{mg}, 142 \mu \mathrm{mol})$ in $\mathrm{CH}_{3} \mathrm{CN}(1.4 \mathrm{~mL})$ at $80{ }^{\circ} \mathrm{C}$ was added PPTS $(0.4 \mathrm{mg}, 1.4 \mu \mathrm{mol})$. After being stirred at $80{ }^{\circ} \mathrm{C}$ for $3 \mathrm{~h}$, the reaction mixture was quenched with $\mathrm{Et}_{3} \mathrm{~N}$ and saturated aqueous $\mathrm{NaHCO}_{3}$, and then extracted with EtOAc (x2). The organic layer was washed with brine, and dried over $\mathrm{Na}_{2} \mathrm{SO}_{4}$. Concentration and flash column 
chromatography (hexane/EtOAc 7:1-0:1) gave 25 $\beta$ (37.9 mg, $103 \mu \mathrm{mol})$ in 74\% yield and recovered $\mathbf{2 5} \alpha(4.6 \mathrm{mg}, 12.6 \mu \mathrm{mol})$ in $9 \%$ yield.

Conversion of $24 \beta$ to $25 \beta$. To a solution of the spiroacetal $24 \beta(10.4 \mathrm{~g}, 28.4 \mathrm{mmol}), \mathrm{Ph}_{3} \mathrm{P}(18.7 \mathrm{~g}, 71$ $\mathrm{mmol})$ and $\mathrm{BzOH}(10 \mathrm{~g}, 85 \mathrm{mmol})$ in toluene $(100 \mathrm{~mL})$ at room temperature was added DEAD (12.4 $\mathrm{g}$, $71 \mathrm{mmol}$ ). After being stirred $1 \mathrm{~h}$ at room temperature, the reaction mixture was quenched with $\mathrm{H}_{2} \mathrm{O}$, and extracted with $\mathrm{Et}_{2} \mathrm{O}(\mathrm{x} 2)$. The organic layer was washed with saturated aqueous $\mathrm{NaHCO}_{3}$ and brine, and dried over $\mathrm{Na}_{2} \mathrm{SO}_{4}$. Concentration and flash column chromatography (hexane/EtOAc 40:1-10:1) gave the benzoate.

To a solution of the benzoate in $\mathrm{MeOH}(30 \mathrm{~mL})$ at room temperature was added $\mathrm{NaOH}(120 \mathrm{mg}, 3$ mmol). After being stirred at room temperature for $24 \mathrm{~h}$, the mixture was quenched with saturated aqueous $\mathrm{NH}_{4} \mathrm{Cl}$, and extracted with EtOAc (x2). The organic layer was washed with aqueous $\mathrm{NaHCO}_{3}$ and brine, and dried over $\mathrm{Na}_{2} \mathrm{SO}_{4}$. Concentration and flash column chromatography (hexane/EtOAc 7:1-0:1) gave $25 \beta(9.5 \mathrm{~g}, 25.9 \mathrm{mmol})$ in $91 \%$ yield over 2 steps.

Alcohol 26. To a solution of spiroacetal $25 \beta(4.30 \mathrm{~g}, 11.7 \mathrm{mmol})$ and 2,6-lutidine $(3.2 \mathrm{~mL}, 28.0$ mmol $)$ in $\left(\mathrm{CH}_{2} \mathrm{Cl}\right)_{2}(11 \mathrm{~mL})$ at $0{ }^{\circ} \mathrm{C}$ was added TIPSOTf $(3.7 \mathrm{~mL}, 14.0 \mathrm{mmol})$. After being stirred at $0{ }^{\circ} \mathrm{C}$ for $2 \mathrm{~h}$, the reaction mixture was quenched with saturated aqueous $\mathrm{NH}_{4} \mathrm{Cl}$, and extracted with hexane/EtOAc (x2). The organic layer was washed with brine and dried over $\mathrm{MgSO}_{4}$. Concentration and flash column chromatography (hexane/EtOAc 0:1-50:1) gave the TIPS ether (5.89 $\mathrm{g}, 11.2 \mathrm{mmol})$ in 96\% yield: colorless oil; $[\alpha]_{\mathrm{D}}^{30}=-33.5\left(c 1.05, \mathrm{CHCl}_{3}\right)$; IR (neat) $v 2942,1460,1381,1146,1091,1019$, 
$921 \mathrm{~cm}^{-1} ;{ }^{1} \mathrm{H}$ NMR (500 MHz, $\left.\mathrm{CDCl}_{3}\right) \delta$ 1.01-1.05 (27H, m, TIPS, Me56, Me57), 1.55 (1H, m, H47), $1.66(1 \mathrm{H}, \mathrm{dq}, J=10.0,6.5 \mathrm{~Hz}, \mathrm{H} 48), 1.98(1 \mathrm{H}, \mathrm{dd}, J=13.5,3.0 \mathrm{~Hz}, \mathrm{H} 50), 2.23$ (1H, dd, $J=13.5,7.0$ Hz, H50), 3.17 (1H, dd, $J=10.0,10.0 \mathrm{~Hz}, \mathrm{H} 46), 3.33$ (3H, s, MOM), 3.57 (1H, dd, $J=11.0,4.5 \mathrm{~Hz}$, H44), 3.61 (1H, dd, $J=11.0,2.5 \mathrm{~Hz}, \mathrm{H} 44), 3.73$ (1H, ddd, $J=10.0,4.5,2.5 \mathrm{~Hz}, \mathrm{H} 45), 3.75(1 \mathrm{H}, \mathrm{d}, J=$ $9.0 \mathrm{~Hz}, \mathrm{H} 52), 3.92(1 \mathrm{H}, \mathrm{dd}, J=9.0,4.5 \mathrm{~Hz}, \mathrm{H} 52), 4.53(1 \mathrm{H}, \mathrm{d}, J=6.0 \mathrm{~Hz}, \mathrm{MOM}), 4.53$ (1H, d, $J=12.0$ Hz, Bn), 4.590 (1H, m, H51), 4.593 (1H, d, $J=12.0 \mathrm{~Hz}, \mathrm{Bn}), 4.67$ (1H, d, $J=6.0 \mathrm{~Hz}, \mathrm{MOM}), 7.26-7.35$ (5H, m, Bn); ${ }^{13} \mathrm{C}$ NMR (125 MHz, $\left.\mathrm{CDCl}_{3}\right) \delta 11.9,13.6,15.7,17.9,39.3,41.9,46.2,56.2,69.5,72.1$, 72.3, 73.2, 75.1, 79.3, 98.1, 108.8, 127.4, 127.8, 128.2, 138.3; MALDI-TOF MS, calcd for $\mathrm{C}_{29} \mathrm{H}_{50} \mathrm{O}_{6} \mathrm{SiNa} 545.32\left(\mathrm{M}+\mathrm{Na}^{+}\right)$, found 545.32.

To a solution of the TIPS ether $(5.89 \mathrm{~g}, 11.2 \mathrm{mmol})$ in EtOAc $(50 \mathrm{~mL})$ at room temperature was added $20 \% \mathrm{Pd}(\mathrm{OH})_{2} / \mathrm{C}(0.31 \mathrm{~g})$, and the mixture was stirred under hydrogen for $4 \mathrm{~d}$. The catalyst was filtered off, and the solution was concentrated to give alcohol $26(4.94 \mathrm{~g}, 11.4 \mathrm{mmol})$ : colorless oil; $[\alpha]_{\mathrm{D}}{ }^{27}=$ $-66.3\left(c\right.$ 1.12, $\left.\mathrm{CHCl}_{3}\right)$; IR (neat) v 3479, 2943, 1463, 1259, 1142, 1098, $950 \mathrm{~cm}^{-1} ;{ }^{1} \mathrm{H} \mathrm{NMR}(500 \mathrm{MHz}$, $\left.\mathrm{CDCl}_{3}\right) \delta 1.01(3 \mathrm{H}, \mathrm{d}, J=6.5 \mathrm{~Hz}, \mathrm{Me} 57), 1.03(21 \mathrm{H}, \mathrm{s}, \mathrm{TIPS}), 1.04(3 \mathrm{H}, \mathrm{d}, J=6.5 \mathrm{~Hz}, \mathrm{Me} 56), 1.52(1 \mathrm{H}$, m, H48), $1.71(1 \mathrm{H}, \mathrm{m}, \mathrm{H} 47), 1.98(1 \mathrm{H}, \mathrm{dd}, J=13.5,3.5 \mathrm{~Hz}, \mathrm{H} 50), 2.21(1 \mathrm{H}, \mathrm{dd}, J=13.5,6.5 \mathrm{~Hz}, \mathrm{H} 50)$, $2.60(1 \mathrm{H}, \mathrm{t}, J=6.0 \mathrm{~Hz}, \mathrm{OH} 44), 3.17(1 \mathrm{H}, \mathrm{dd}, J=10.0,10.0 \mathrm{~Hz}, \mathrm{H} 46), 3.43(3 \mathrm{H}, \mathrm{s}, \mathrm{MOM}), 3.61-3.67$ $(2 \mathrm{H}, \mathrm{m}, \mathrm{H} 44,45), 3.76(1 \mathrm{H}, \mathrm{d}, J=9.5 \mathrm{~Hz}, \mathrm{H} 52), 3.81(1 \mathrm{H}, \mathrm{m}, \mathrm{H} 44), 3.92(1 \mathrm{H}, \mathrm{dd}, J=9.5,4.0 \mathrm{~Hz}, \mathrm{H} 52)$, $4.58(1 \mathrm{H}, \mathrm{m}, \mathrm{H} 51), 4.69(1 \mathrm{H}, \mathrm{d}, J=6.5 \mathrm{~Hz}, \mathrm{MOM}), 4.71(1 \mathrm{H}, \mathrm{d}, J=6.5 \mathrm{~Hz}, \mathrm{MOM}) ;{ }^{13} \mathrm{C}$ NMR $(125$ $\left.\mathrm{MHz}, \mathrm{CDCl}_{3}\right) \delta 11.9,13.5,15.4,17.8,39.5,42.0,46.2,56.3,62.3,72.0,72.9,75.1,79.6,98.8,109.0 ;$ HRMS (FAB), calcd for $\mathrm{C}_{22} \mathrm{H}_{44} \mathrm{O}_{6} \mathrm{SiNa} 455.2805\left(\mathrm{M}+\mathrm{Na}^{+}\right)$, found 455.2808. 
Homoallylic alcohol 28. A solution of $(\mathrm{COCl})_{2}(25.8 \mathrm{~mL}, 290 \mathrm{mmol})$ in $\mathrm{CH}_{2} \mathrm{Cl}_{2}(728 \mathrm{~mL})$ was treated with DMSO $(25.9 \mathrm{~mL}, 365 \mathrm{mmol})$ at $-80{ }^{\circ} \mathrm{C}$, and the resulting solution was stirred at $-80{ }^{\circ} \mathrm{C}$ to $-60{ }^{\circ} \mathrm{C}$ for $30 \mathrm{~min}$. To the solution was added alcohol $26(31.5 \mathrm{~g}, 72.8 \mathrm{mmol})$ at $-60{ }^{\circ} \mathrm{C}$. After being stirred at $-60{ }^{\circ} \mathrm{C}$ for $30 \mathrm{~min}$, the mixture was treated with $\mathrm{Et}_{3} \mathrm{~N}(80.7 \mathrm{~mL}, 580 \mathrm{mmol})$ at $-60{ }^{\circ} \mathrm{C}$ and allowed to warm to $-30{ }^{\circ} \mathrm{C}$ over $1 \mathrm{~h}$. The reaction mixture was then quenched with saturated aqueous $\mathrm{NH}_{4} \mathrm{Cl}$, and extracted with EtOAc (x2). The organic layer was washed with saturated aqueous $\mathrm{NH}_{4} \mathrm{Cl}$ and brine, and dried over $\mathrm{MgSO}_{4}$. Concentration gave aldehyde 27, which was used in the next reaction without further purification.

To a solution of aldehyde 27 in toluene $(360 \mathrm{~mL})$ at $-80{ }^{\circ} \mathrm{C}$ was added $(R, R)$-diisopropyl tartrate $(\mathrm{Z})$ crotylboronate $(\mathbf{1 9}, 1.0 \mathrm{M}$ in toluene, $146 \mathrm{~mL}, 146 \mathrm{mmol})$. After being stirred at $-80{ }^{\circ} \mathrm{C}$ for $1 \mathrm{~h}$, the reaction mixture was quenched with $15 \% \mathrm{NaOH}$ solution and stirred at room temperature for $1 \mathrm{~h}$. The solution was extracted with EtOAc (x2), and the organic layer was washed with brine, and dried over $\mathrm{MgSO}_{4}$. Concentration and flash column chromatography (hexane/EtOAc 50:1-1:1) gave homoallylic alcohol $28(27.9 \mathrm{~g}, 57.3 \mathrm{mmol})$ in $79 \%$ yield over 2 steps: colorless oil; $[\alpha]_{\mathrm{D}}{ }^{28}=-71.2\left(c 1.06, \mathrm{CHCl}_{3}\right)$; IR (neat) v 3529, 2943, 1382, 1258, 1141, 1018, $941 \mathrm{~cm}^{-1} ;{ }^{1} \mathrm{H}$ NMR $\left(500 \mathrm{MHz}, \mathrm{CDCl}_{3}\right) \delta 0.99-1.03$ (30H, m, TIPS, Me55, Me56, Me57), 1.49 (1H, dq, $J=11.0,6.5$ Hz, H48), 1.74 (1H, ddq, $J=11.0,10.0$, $6.5 \mathrm{~Hz}, \mathrm{H} 47), 1.98(1 \mathrm{H}, \mathrm{dd}, J=13.5,3.5 \mathrm{~Hz}, \mathrm{H} 50), 2.12(1 \mathrm{H}, \mathrm{dd}, J=13.5,7.0 \mathrm{~Hz}, \mathrm{H} 50), 2.59(1 \mathrm{H}, \mathrm{m}$, H43), 3.18 (1H, dd, $J=10.0,10.0 \mathrm{~Hz}, \mathrm{H} 46), 3.33$ (1H, d, $J=5.0 \mathrm{~Hz}, \mathrm{OH} 44), 3.40$ (3H, s, MOM), 3.62 $(1 \mathrm{H}, \mathrm{ddd}, J=5.0,5.0,5.0 \mathrm{~Hz}, \mathrm{H} 44), 3.72(1 \mathrm{H}, \mathrm{dd}, J=10.0,5.0 \mathrm{~Hz}, \mathrm{H} 45), 3.75(1 \mathrm{H}, \mathrm{dd}, J=9.0,2.0 \mathrm{~Hz}$ H52), 3.93 (1H, dd, J=9.0, $4.0 \mathrm{~Hz}, \mathrm{H} 52), 4.59$ (1H, m, H51), 4.69 (1H, d, J = 6.0 Hz, MOM), 4.79 (1H, $\mathrm{d}, J=6.0 \mathrm{~Hz}, \mathrm{MOM}), 5.03(1 \mathrm{H}, \mathrm{dd}, J=10.0,1.0 \mathrm{~Hz}, \mathrm{H} 41), 5.06(1 \mathrm{H}, \mathrm{dd}, J=17.5,1.0 \mathrm{~Hz}, \mathrm{H} 41), 5.91$ 
$(1 \mathrm{H}, \mathrm{ddd}, J=17.5,10.0,7.5 \mathrm{~Hz}, \mathrm{H} 42) ;{ }^{13} \mathrm{C} \mathrm{NMR}\left(125 \mathrm{MHz}, \mathrm{CDCl}_{3}\right) \delta 11.9,13.0,13.4,15.4,17.8,38.9$ 40.1, 42.2, 46.1, 56.1, 71.6, 71.9, 75.3, 76.7, 84.5, 98.9, 108.5, 113.8, 142.6; MALDI-TOF MS, calcd for $\mathrm{C}_{26} \mathrm{H}_{50} \mathrm{O}_{6} \mathrm{SiNa} 509.32\left(\mathrm{M}+\mathrm{Na}^{+}\right)$, found 509.33 .

NAP ether 29. To a solution of alcohol 28 (1.45 g, $2.97 \mathrm{mmol})$ in THF at room temperature was added TBAF (1.0 M solution of in THF, $3.27 \mathrm{~mL}, 3.27 \mathrm{mmol}$ ). After being stirred for $4 \mathrm{~h}$, the mixture was directly subjected to column chromatography (hexane/EtOAc 2:1) to give the diol, which was used in the next reaction without further purification.

To a solution of the diol in THF/DMF $(1: 1,6.8 \mathrm{~mL})$ at $0{ }^{\circ} \mathrm{C}$ were sequentially added $\mathrm{NaH}(1.02 \mathrm{~g}, 12.8$ mmol), NAPBr $(2.83 \mathrm{~g}, 12.8 \mathrm{mmol})$ and TBAI $(0.59 \mathrm{~g}, 1.60 \mathrm{mmol})$. After $10 \mathrm{~min}$, additional $\mathrm{NaH}$ $(1.02 \mathrm{~g}, 12.8 \mathrm{mmol})$ were introduced to the mixture. After being stirred at room temperature for $5 \mathrm{~h}$, the solution was treated with $\mathrm{MeOH}$ and saturated aqueous $\mathrm{NH}_{4} \mathrm{Cl}$, and extracted with $\mathrm{Et}_{2} \mathrm{O}(\mathrm{x} 2)$. The organic layer was washed with $\mathrm{H}_{2} \mathrm{O}(\mathrm{x} 2)$ and brine, and dried over $\mathrm{MgSO}_{4}$. Concentration and column chromatography (hexane/EtOAc 30:1) gave bis-NAP ether 29 (1.81 g, 2.96 mmol) in 99\% over 2 steps: pale yellow oil; $[\alpha]_{\mathrm{D}}{ }^{21}=-31.6\left(c 1.89, \mathrm{CHCl}_{3}\right) ; \mathrm{IR}($ film $) \vee 3055,2974,2927,1457,1093,938 \mathrm{~cm}^{-1} ;{ }^{1} \mathrm{H}$ $\operatorname{NMR}\left(500 \mathrm{MHz}, \mathrm{CDCl}_{3}\right) \delta 1.03(3 \mathrm{H}, \mathrm{d}, J=6.5 \mathrm{~Hz}, \mathrm{Me} 57), 1.05(3 \mathrm{H}, \mathrm{d}, J=6.0 \mathrm{~Hz}, \mathrm{Me} 56), 1.09(3 \mathrm{H}, \mathrm{d}$, $J=7.0 \mathrm{~Hz}, \mathrm{Me} 55), 1.52$ (1H, m, H48), 1.69 (1H, m, H47), 2.11 (1H, dd, $J=13.5,3.5 \mathrm{~Hz}, \mathrm{H} 50), 2.15$ $(1 \mathrm{H}, \mathrm{dd}, J=13.5,6.5 \mathrm{~Hz}, \mathrm{H} 50), 2.60(1 \mathrm{H}, \mathrm{m}, \mathrm{H} 43), 3.27(1 \mathrm{H}, \mathrm{dd}, J=10.0,10.0 \mathrm{~Hz}, \mathrm{H} 46), 3.34$ (1H, d, $J=8.5 \mathrm{~Hz}, \mathrm{H} 44), 3.36(3 \mathrm{H}, \mathrm{s}, \mathrm{MOM}), 3.88(1 \mathrm{H}, \mathrm{dd}, J=10.0,5.0 \mathrm{~Hz}, \mathrm{H} 52), 3.99(1 \mathrm{H}, \mathrm{d}, J=10.0 \mathrm{~Hz}$ H45), $4.01(1 \mathrm{H}, \mathrm{d}, J=10.0 \mathrm{~Hz}, \mathrm{H} 52), 4.33(1 \mathrm{H}, \mathrm{m}, \mathrm{H} 51), 4.61(2 \mathrm{H}, \mathrm{s}, \mathrm{NAP}), 4.62(1 \mathrm{H}, \mathrm{d}, J=6.0 \mathrm{~Hz}$ MOM), $4.64(1 \mathrm{H}, \mathrm{d}, J=11.0 \mathrm{~Hz}, \mathrm{NAP}), 4.79(1 \mathrm{H}, \mathrm{d}, J=6.0 \mathrm{~Hz}, \mathrm{MOM}), 4.87(1 \mathrm{H}, \mathrm{d}, J=11.0 \mathrm{~Hz}$ 
NAP), $4.98(1 \mathrm{H}, \mathrm{dd}, J=10.0,2.0 \mathrm{~Hz}, \mathrm{H} 41), 5.04(1 \mathrm{H}, \mathrm{dd}, J=17.0,2.0 \mathrm{~Hz}, \mathrm{H} 41), 5.75(1 \mathrm{H}, \mathrm{ddd}, J=$ 17.5, 10.0, 5.0 Hz, H42), 7.43-7.48 (6H, m, NAPx2), 7.76-7.83 (8H, m, NAPx2); ${ }^{13} \mathrm{C}$ NMR (125 MHz, $\left.\mathrm{CDCl}_{3}\right) \delta 13.6,15.8,17.5,39.9,40.1,41.8,42.5,56.2,71.1,71.3,72.9,73.2,78.6,80.4,83.2,98.4$, $108.5,114.7,125.62,125.65,125.8,125.9,126.0,126.1,126.2,126.4,127.62,127.65,127.79,127.81$, $127.82,128.1,132.8,132.9,133.1,133.2,135.6,136.3,142.1$; MALDI-TOF MS, calcd for $\mathrm{C}_{39} \mathrm{H}_{46} \mathrm{O}_{6} \mathrm{Na}$ $633.32\left(\mathrm{M}+\mathrm{Na}^{+}\right)$, found, 633.32; Anal. calcd for $\mathrm{C}_{39} \mathrm{H}_{46} \mathrm{O}_{6}: \mathrm{C}, 76.69, \mathrm{H}, 7.59$, found $\mathrm{C}, 76.72, \mathrm{H}, 7.96$.

Carboxylic acid 11. To a solution of NAP ether $29(1.81 \mathrm{~g}, 2.96 \mathrm{mmol})$ and NMO (1.85 mL, 8.89 mmol) in $t$ - $\mathrm{BuOH} / \mathrm{H}_{2} \mathrm{O}$ /acetone $(1: 1: 1,22 \mathrm{~mL})$ at room temperature was added $\mathrm{OsO}_{4}(38 \mathrm{mM}$ in $t-\mathrm{BuOH}$, $0.76 \mathrm{~mL}, 0.03 \mathrm{mmol})$. After $8 \mathrm{~h}$, additional $\mathrm{NMO}(1.85 \mathrm{~mL}, 8.89 \mathrm{mmol})$ was introduced, and the resulting mixture was stirred for $2 \mathrm{~d}$. The solution was then treated with $\mathrm{NaIO}_{4}(1.26 \mathrm{~g}, 5.92 \mathrm{mmol})$ and stirred for $7 \mathrm{~h}$. The reaction mixture was quenched with saturated aqueous $\mathrm{Na}_{2} \mathrm{~S}_{2} \mathrm{O}_{3}$, and extracted with $\mathrm{Et}_{2} \mathrm{O}$ (x3). The organic layer was washed with brine, dried over $\mathrm{MgSO}_{4}$, and concentrated to give crude aldehyde, which was used in the next reaction without further purification.

To a solution of the aldehyde in $t-\mathrm{BuOH} / \mathrm{H}_{2} \mathrm{O}(5: 1,30 \mathrm{~mL})$ at room temperature were added $\mathrm{NaH}_{2} \mathrm{PO}_{4} \cdot 2 \mathrm{H}_{2} \mathrm{O}(0.92 \mathrm{~g}, 5.92 \mathrm{mmol})$, 2-methyl-2-butene $(1.5 \mathrm{~mL}, 14.8 \mathrm{mmol})$, and $\mathrm{NaClO}_{2}(0.94 \mathrm{~g}$, $10.36 \mathrm{mmol}$ ). After being stirred at room temperature for $7 \mathrm{~h}$, the mixture was diluted with EtOAc. The organic layer was washed with brine, and dried over $\mathrm{MgSO}_{4}$. Concentration and column chromatography (hexane/EtOAc 2:1) gave carboxylic acid $\mathbf{1 1}$ (1.52 g, $2.41 \mathrm{mmol}$ ) in 82\% yield over 2 steps: pale yellow oil; $[\alpha]_{\mathrm{D}}^{22}=-40.2\left(c 1.34, \mathrm{CHCl}_{3}\right)$; IR (film) v 3055, 2976, 1705, 1458, 1174, 1028, $940 \mathrm{~cm}^{-1} ;{ }^{1} \mathrm{H}$ NMR $\left(500 \mathrm{MHz}, \mathrm{CDCl}_{3}\right) \delta 1.02(3 \mathrm{H}, \mathrm{d}, J=6.5 \mathrm{~Hz}, \mathrm{Me} 57), 1.03(3 \mathrm{H}, \mathrm{d}, J=6.0 \mathrm{~Hz}, \mathrm{Me} 56)$, 
$1.30(3 \mathrm{H}, \mathrm{d}, J=6.0 \mathrm{~Hz}, \mathrm{Me} 55), 1.52(1 \mathrm{H}, \mathrm{m}, \mathrm{H} 48), 1.73(1 \mathrm{H}, \mathrm{m}, \mathrm{H} 47), 2.08(1 \mathrm{H}, \mathrm{dd}, J=14.0,3.5 \mathrm{~Hz}$, H50), 2.19 (1H, dd, $J=14.0,7.0 \mathrm{~Hz}, \mathrm{H} 50), 2.86$ (1H, m, H43), 3.09 (1H, dd, $J=10.0,10.0 \mathrm{~Hz}, \mathrm{H} 46)$, $3.29(3 \mathrm{H}, \mathrm{s}, \mathrm{MOM}), 3.93(1 \mathrm{H}, \mathrm{dd}, J=10.0,5.0 \mathrm{~Hz}, \mathrm{H} 52), 4.00(1 \mathrm{H}, \mathrm{d}, J=10.0 \mathrm{~Hz}, \mathrm{H} 52) 4.04(1 \mathrm{H}, \mathrm{d}, J$ $=10.0 \mathrm{~Hz}, \mathrm{H} 45), 4.09(1 \mathrm{H}, \mathrm{d}, J=6.0 \mathrm{~Hz}, \mathrm{H} 44), 4.37(1 \mathrm{H}, \mathrm{m}, \mathrm{H} 51), 4.59(2 \mathrm{H}, \mathrm{s}, \mathrm{NAP}), 4.63(1 \mathrm{H}, \mathrm{d}, J=$ $6.5 \mathrm{~Hz}, \mathrm{MOM}), 4.71(1 \mathrm{H}, \mathrm{d}, J=6.5 \mathrm{~Hz}, \mathrm{MOM}), 4.74(1 \mathrm{H}, \mathrm{d}, J=12.0 \mathrm{~Hz}, \mathrm{NAP}), 4.90(1 \mathrm{H}, \mathrm{d}, J=12.0$

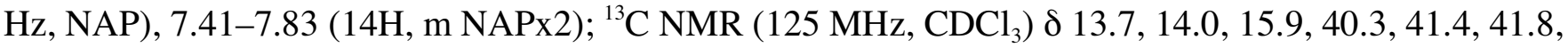
$42.4,56.4,71.4,71.9,72.7,74.1,78.8,79.3,81.3,98.8,108.9,125.9,126.0,126.1,126.2,126.3,126.4$, $126.8,127.8,127.9,128.0,128.3,128.4,133.1,133.2,133.41,133.47,135.50,135.8,178.6$; HRMS (FAB), calcd for $\mathrm{C}_{38} \mathrm{H}_{44} \mathrm{O}_{8} \mathrm{Na} 651.2934\left(\mathrm{M}+\mathrm{Na}^{+}\right)$, found 651.2929 .

Ketone 32. To a suspension of diol $5(7.31 \mathrm{~g}, 21.6 \mathrm{mmol})$ and $\mathrm{MS} 4 \mathrm{~A}(5.0 \mathrm{~g})$ in $\mathrm{CH}_{2} \mathrm{Cl}_{2}(430 \mathrm{~mL})$ at $-78{ }^{\circ} \mathrm{C}$ were added 2,6-lutidine $(12.6 \mathrm{~mL}, 108 \mathrm{mmol})$ and $\mathrm{Tf}_{2} \mathrm{O}(3.65 \mathrm{~mL}, 21.6 \mathrm{mmol})$. After being stirred at $-78{ }^{\circ} \mathrm{C}$ for $25 \mathrm{~min}$, the mixture was treated with TESOTf $(12.2 \mathrm{~mL}, 54 \mathrm{mmol})$, and stirred for 30 min. The reaction mixture was quenched with saturated aqueous $\mathrm{NH}_{4} \mathrm{Cl}$, and extracted with $\mathrm{Et}_{2} \mathrm{O}$ (x2). The organic layer was washed with $\mathrm{NaHCO}_{3}$ and brine, and dried over $\mathrm{Na}_{2} \mathrm{SO}_{4}$. Concentration gave triflate 30, which was subjected to the next reaction immediately.

To a solution of triflate 30, epoxysulfone 8 (10.9 g, $22.7 \mathrm{mmol})$ and HMPA (15 mL, $86.4 \mathrm{mmol})$ in THF $(220 \mathrm{~mL})$ at $-100{ }^{\circ} \mathrm{C}$ was added $n$-BuLi (1.55 M solution in hexane, $\left.14.6 \mathrm{~mL}, 22.7 \mathrm{mmol}\right)$. After being stirred at $-100{ }^{\circ} \mathrm{C}$ for $40 \mathrm{~min}$, the reaction mixture was quenched with saturated aqueous $\mathrm{NH}_{4} \mathrm{Cl}$. The mixture was extracted with EtOAc (x2), washed with brine (x2), and dried over $\mathrm{MgSO}_{4}$. Concentration and column chromatography (hexane/EtOAc 30:1-10:1) gave epoxysulfone 31, which 
was used in the next reaction without further purification.

To a solution of epoxy sulfone 31 in $\mathrm{CHCl}_{3}(380 \mathrm{~mL})$ was added $p$ - $\mathrm{TsOH} \cdot \mathrm{H}_{2} \mathrm{O}(5.4 \mathrm{~g}, 28.2 \mathrm{mmol})$, and the resulting mixture was stirred at $40{ }^{\circ} \mathrm{C}$ for $2 \mathrm{~h}$. The reaction mixture was quenched with $\mathrm{Et}_{3} \mathrm{~N}$ and extracted with EtOAc (x2). The organic layer was washed with saturated aqueous $\mathrm{NaHCO}_{3}$ and brine, and dried over $\mathrm{Na}_{2} \mathrm{SO}_{4}$. Concentration and column chromatography (hexane/EtOAc 30:1-8:1) gave ketone $32(7.36 \mathrm{~g}, 11.0 \mathrm{mmol})$ in $51 \%$ yield over 3 steps: colorless oil; $[\alpha]_{\mathrm{D}}{ }^{30}=-15.3\left(c 1.18, \mathrm{CHCl}_{3}\right)$; IR (neat) v 2930, 1723, 1615, 1517, 1249, 1112, $825 \mathrm{~cm}^{-1} ;{ }^{1} \mathrm{H}$ NMR $\left(500 \mathrm{MHz}, \mathrm{CDCl}_{3}\right) \delta 1.02(9 \mathrm{H}, \mathrm{s}$ TBPS), 1.16 (3H, d, $J=7.5$ Hz, Me54), 1.17 (3H, s, Me53), 1.74-1.85 (2H, m, H35, H37), 1.96-2.04 (3H, m, H35, H36, H37), 2.43 (1H, dd, $J=17.0,7.5 \mathrm{~Hz}, \mathrm{H} 32), 3.04$ (1H, dd, $J=17.5,7.5 \mathrm{~Hz}, \mathrm{H} 32)$, 3.49-3.54 (3H, m, H34, H39, H40), 3.60 (1H, d, J = 9.5 Hz, H29), 3.62 (1H, m, H38), 3.80 (3H, s, MP), $3.81(1 \mathrm{H}, \mathrm{d}, J=9.5 \mathrm{~Hz}, \mathrm{H} 29), 3.86(1 \mathrm{H}, \mathrm{ddd}, J=7.5,7.5,7.5 \mathrm{~Hz}, \mathrm{H} 33), 4.15(1 \mathrm{H}, \mathrm{dd}, J=5.0,2.5 \mathrm{~Hz}$ H40), 5.37 (1H, s, MP), 6.88 (2H, d, $J=6.5 \mathrm{~Hz}, \mathrm{MP}), 7.23-7.73(12 \mathrm{H}, \mathrm{m}, \mathrm{TBPS}, \mathrm{MP}) ;{ }^{13} \mathrm{C}$ NMR $(125$ $\left.\mathrm{MHz}, \mathrm{CDCl}_{3}\right) \delta 17.3,19.2,26.6,26.8,27.7,28.7,45.2,46.2,55.2,69.7,75.4,78.6,81.7,82.8,84.4$ $100.7,113.6,127.3,127.5,127.6,129.64,129.69,130.1,133.0,133.1,135.6,135.7,159.9,211.3$; HRMS (FAB), calcd for $\mathrm{C}_{38} \mathrm{H}_{48} \mathrm{O}_{7} \mathrm{SiNa} 667.3067\left(\mathrm{M}+\mathrm{Na}^{+}\right)$, found 667.3051 .

TIPS ether 33. To a solution of ketone $32(0.88 \mathrm{~g}, 1.36 \mathrm{mmol})$ in $\mathrm{CH}_{2} \mathrm{Cl}_{2} / \mathrm{MeOH}(1: 1,13.6 \mathrm{~mL})$ at $-78{ }^{\circ} \mathrm{C}$ was added $\mathrm{NaBH}_{4}(0.10 \mathrm{~g}, 2.72 \mathrm{mmol})$. After being stirred for $40 \mathrm{~min}$, the reaction mixture was quenched with saturated aqueous $\mathrm{NH}_{4} \mathrm{Cl}$, and extracted with EtOAc (x2). The organic layer was washed with brine, and dried over $\mathrm{MgSO}_{4}$. Concentration and column chromatography (hexane/EtOAc 1:2) gave the alcohol $(0.89 \mathrm{~g}, 1.37 \mathrm{mmol})$ in $100 \%$ yield: colorless oil; $[\alpha]_{\mathrm{D}}^{28}=2.90(c$ 
1.03, $\left.\mathrm{CHCl}_{3}\right) ; \mathrm{IR}($ film $) \vee 3531,2931,1615,1516,1250,1082,827 \mathrm{~cm}^{-1} ;{ }^{1} \mathrm{H} \mathrm{NMR}\left(500 \mathrm{MHz}, \mathrm{CDCl}_{3}\right) \delta$

$1.03(3 \mathrm{H}, \mathrm{d}, J=7.0 \mathrm{~Hz}, \mathrm{Me} 54), 1.06(9 \mathrm{H}, \mathrm{s}, \mathrm{TBPS}), 1.29(3 \mathrm{H}, \mathrm{s}, \mathrm{Me} 53), 1.48(1 \mathrm{H}, \mathrm{ddd}, J=14.5,9.0$, $7.0 \mathrm{~Hz}, \mathrm{H} 35), 1.65$ (1H, m, H35), 1.63 (1H, ddd, $J=12.0,12.0,12.0 \mathrm{~Hz}, \mathrm{H} 32), 1.68(1 \mathrm{H}, \mathrm{dd}, J=14.5$, $3.5 \mathrm{~Hz}, \mathrm{H} 37), 1.86(1 \mathrm{H}, \mathrm{dq}, J=7.0,7.0 \mathrm{~Hz}, \mathrm{H} 36), 1.93(1 \mathrm{H}, \mathrm{dd}, J=14.5,3.5 \mathrm{~Hz}, \mathrm{H} 37), 2.12$ (1H, ddd, $J$ $=12.0,5.0,5.0 \mathrm{~Hz}, \mathrm{H} 32), 3.17(1 \mathrm{H}, \mathrm{ddd}, J=12.0,9.0,5.0 \mathrm{~Hz}, \mathrm{H} 33), 3.32(1 \mathrm{H}, \mathrm{ddd}, J=9.0,9.0,3.0 \mathrm{~Hz}$, H34), $3.33(1 \mathrm{H}, \mathrm{d}, J=1.0 \mathrm{~Hz}, \mathrm{OH} 31), 3.42(1 \mathrm{H}, \mathrm{ddd}, J=11.0,11.0,5.5 \mathrm{~Hz}, \mathrm{H} 39), 3.51(1 \mathrm{H}, \mathrm{dd}, J=$ 11.0, 11.0 Hz, H40), 3.56 (2H, s, H29x2), 3.57 (1H, ddd, J = 11.0, 11.0, $3.5 \mathrm{~Hz}, \mathrm{H} 38), 3.78$ (3H, s, MP), $3.83(1 \mathrm{H}, \mathrm{ddd}, J=12.0,5.0,1.0 \mathrm{~Hz}, \mathrm{H} 31), 4.18(1 \mathrm{H}, \mathrm{dd}, J=11.0,5.5 \mathrm{~Hz}, \mathrm{H} 40), 5.36(1 \mathrm{H}, \mathrm{s}, \mathrm{MP}), 6.86$ $(2 \mathrm{H}, \mathrm{d}, J=8.0 \mathrm{~Hz}, \mathrm{MP}), 7.23-7.44(10 \mathrm{H}, \mathrm{m}, \mathrm{TBPS}), 7.65(2 \mathrm{H}, \mathrm{d}, J=8.0 \mathrm{~Hz}, \mathrm{MP}) ;{ }^{13} \mathrm{C} \mathrm{NMR}(125 \mathrm{MHz}$ $\left.\mathrm{CDCl}_{3}\right) \delta 13.5,19.1,26.8,27.9,28.2,36.2,45.9,55.2,70.0,72.0,73.3,73.7,74.8,79.2,81.2,84.2$ $100.6,113.6,127.3,127.8,129.9,130.0,130.2,132.1,132.3,135.5,135.6,159.9$; HRMS (MALDI), calcd for $\mathrm{C}_{38} \mathrm{H}_{50} \mathrm{O}_{7} \mathrm{SiNa} 669.3223\left(\mathrm{M}+\mathrm{Na}^{+}\right)$, found 669.3215 .

To a solution of the alcohol $(1.06 \mathrm{~g}, 1.63 \mathrm{mmol})$ and 2,6 -lutidine $(0.45 \mathrm{~mL}, 3.93 \mathrm{mmol})$ in $\left(\mathrm{CH}_{2} \mathrm{Cl}\right)_{2}(6.5$ $\mathrm{mL})$ at room temperature was added TIPSOTf $(0.52 \mathrm{~mL}, 1.95 \mathrm{mmol})$, and the resulting mixture was stirred at $50{ }^{\circ} \mathrm{C}$ for $3 \mathrm{~h}$. Additional reagents [2,6-lutidine $(0.075 \mathrm{~mL}, 0.65 \mathrm{mmol})$ and TIPSOTf $(0.086$ $\mathrm{mL}, 0.32 \mathrm{mmol})]$ were introduced to the mixture. After $20 \mathrm{~min}$ at $50{ }^{\circ} \mathrm{C}$, the reaction mixture was quenched with saturated aqueous $\mathrm{NH}_{4} \mathrm{Cl}$, and extracted with hexane/EtOAc (x2). The organic layer was washed with brine, and dried over $\mathrm{MgSO}_{4}$. Concentration and flash column chromatography (hexane/EtOAc 1:0-10:1) gave TIPS ether $33(1.34 \mathrm{~g}, 1.63 \mathrm{mmol})$ in $100 \%$ yield: colorless oil; $[\alpha]_{\mathrm{D}}{ }^{29}=$ $3.12\left(c\right.$ 1.17, $\left.\mathrm{CHCl}_{3}\right)$; IR (film) v 2931, 1516, 1462, 1249, 1105, $826 \mathrm{~cm}^{-1} ;{ }^{1} \mathrm{H} \mathrm{NMR}\left(500 \mathrm{MHz}, \mathrm{CDCl}_{3}\right)$ ઈ 1.03-1.11 (36H, m, TBPS, TIPS, Me53, Me54), 1.52-1.59 (2H, m, H35, H37), 1.63-1.73 (2H, m, H32, 
H35), 1.85 (1H, dd, $J=11.0,3.0 \mathrm{~Hz}, \mathrm{H} 37), 1.99$ (1H, m, H36), 2.10 (1H, ddd, $J=12.0,5.0,5.0 \mathrm{~Hz}$, H32), 3.14 (1H, ddd, $J=12.0,10.0,5.0 \mathrm{~Hz}, \mathrm{H} 33), 3.34$ (1H, ddd, $J=10.0,10.0,2.5 \mathrm{~Hz}, \mathrm{H} 34), 3.47$ (1H, ddd, $J=10.0,10.0,2.5 \mathrm{~Hz}, \mathrm{H} 39), 3.53(1 \mathrm{H}, \mathrm{dd}, J=10.0,10.0 \mathrm{~Hz}, \mathrm{H} 40), 3.56(1 \mathrm{H}, \mathrm{d}, J=10.5 \mathrm{~Hz}, \mathrm{H} 29)$, $3.60(1 \mathrm{H}, \mathrm{ddd}, J=10.0,8.5,3.0 \mathrm{~Hz}, \mathrm{H} 38), 3.70(1 \mathrm{H}, \mathrm{d}, J=10.5 \mathrm{~Hz}, \mathrm{H} 29), 3.80(3 \mathrm{H}, \mathrm{s}, \mathrm{MP}), 4.11(1 \mathrm{H}$, $\mathrm{dd}, J=12.0,5.0 \mathrm{~Hz}, \mathrm{H} 31), 4.20(1 \mathrm{H}, \mathrm{dd}, J=10.0,5.0 \mathrm{~Hz}, \mathrm{H} 40), 5.38(1 \mathrm{H}, \mathrm{s}, \mathrm{MP}), 6.87(2 \mathrm{H}, \mathrm{d}, J=7.0$ $\mathrm{Hz}, \mathrm{MP}), 7.32-7.75(12 \mathrm{H}, \mathrm{m}, \mathrm{TBPS}, \mathrm{MP}) ;{ }^{13} \mathrm{C} \mathrm{NMR}\left(125 \mathrm{MHz}, \mathrm{CDCl}_{3}\right) \delta 12.2,12.5,12.7,17.6,18.18$, $18.19,19.3,26.8,27.9,28.3,38.0,46.0,46.2,55.2,67.3,68.3,70.1,74.0,77.8,79.1,81.4,84.5,100.7$, 113.6, 127.2, 127.3, 127.4, 129.3, 130.3, 133.7, 134.2, 135.7, 136.0, 159.9; HRMS (FAB), calcd for $\mathrm{C}_{47} \mathrm{H}_{70} \mathrm{O}_{7} \mathrm{Si}_{2} \mathrm{Na} 825.4558\left(\mathrm{M}+\mathrm{Na}^{+}\right)$, found 825.4551 .

Diol 34. To a solution of TIPS ether $33(160 \mathrm{mg}, 0.20 \mathrm{mmol})$ in trifluoroacetic acid/THF/ $\mathrm{H}_{2} \mathrm{O}(1: 10: 5$, $1.2 \mathrm{~mL}$ ) was stirred at $0{ }^{\circ} \mathrm{C}$, and the resulting mixture was stirred at room temperature for $25 \mathrm{~h}$. The reaction mixture was quenched with aqueous $15 \% \mathrm{NaOH}$, and extracted with EtOAc (x3). The organic layer was washed with brine (x2), and dried over $\mathrm{MgSO}_{4}$. Concentration and chromatography gave diol 34 (119 mg, $0.17 \mathrm{mmol})$ in $87 \%$ yield: white solid; m.p. $116-118{ }^{\circ} \mathrm{C}$; $[\alpha]_{\mathrm{D}}{ }^{21}=-1.47(c 1.05$, $\left.\mathrm{CHCl}_{3}\right) ; \mathrm{IR}\left(\right.$ film) v 3379, 2943, 1462, 1109, 1067, $883 \mathrm{~cm}^{-1} ;{ }^{1} \mathrm{H}$ NMR $\left(500 \mathrm{MHz}, \mathrm{CDCl}_{3}\right) \delta 1.02-1.06$ (33H, m, TIPS, TBPS, Me53), 1.09 (3H, d, $J=7.0 \mathrm{~Hz}, \mathrm{Me} 54), 1.51$ (1H, m, H35), 1.66-1.74 (2H, m, H32, H37), 1.80-1.92 (3H, m, H35, H36, H37), 2.17 (1H, ddd, J = 12.0, 5.0, 5.0 Hz, H32), 3.19 (1H, ddd, $J=12.0,10.0,5.0 \mathrm{~Hz}, \mathrm{H} 33), 3.34(1 \mathrm{H}, \mathrm{ddd}, J=10.0,10.0,3.0 \mathrm{~Hz}, \mathrm{H} 34), 3.39(1 \mathrm{H}, \mathrm{dd}, J=10.0$, $5.0 \mathrm{~Hz}, \mathrm{H} 40), 3.55(1 \mathrm{H}, \mathrm{d}, J=10.5 \mathrm{~Hz}, \mathrm{H} 29), 3.64-3.69(2 \mathrm{H}, \mathrm{m}, \mathrm{H} 38, \mathrm{H} 40), 3.68(1 \mathrm{H}, \mathrm{d}, J=10.5 \mathrm{~Hz}$, H29), 3.83 (1H, m, H39), 4.08 (1H, dd, $J=12.0,5.0 \mathrm{~Hz}, \mathrm{H} 31), 7.31-7.74(10 \mathrm{H}, \mathrm{m}, \mathrm{TBPS}) ;{ }^{13} \mathrm{C}$ NMR 
$\left(125 \mathrm{MHz}, \mathrm{CDCl}_{3}\right) \delta 12.4,12.7,18.18,18.19,19.3,26.8,27.90,27.94,37.6,45.5,47.7,65.3,67.2,68.4$ 73.1, 73.2, 77.7, 83.5, 87.1, 127.2, 127.3, 129.3, 133.7, 134.2, 135.7, 136.0; MALDI-TOF MS, calcd for $\mathrm{C}_{39} \mathrm{H}_{64} \mathrm{O}_{6} \mathrm{Si}_{2} \mathrm{Na} 707.41\left(\mathrm{M}+\mathrm{Na}^{+}\right)$, found 707.47; Anal. calcd for $\mathrm{C}_{39} \mathrm{H}_{64} \mathrm{O}_{6} \mathrm{Si}_{2}: \mathrm{C}, 68.37, \mathrm{H}, 9.42$, found $\mathrm{C}$, 68.23, H, 9.62.

Nitrile 35. To a suspension of diol $34(457 \mathrm{mg}, 0.66 \mathrm{mmol})$ and MS4A in THF $(3.3 \mathrm{~mL})$ at $-30{ }^{\circ} \mathrm{C}$ were added imidazole (89 mg, $1.32 \mathrm{mmol}), \mathrm{PPh}_{3}(191 \mathrm{mg}, 0.73 \mathrm{mmol})$ and $\mathrm{I}_{2}(167 \mathrm{mg}, 0.66 \mathrm{mmol})$. After $20 \mathrm{~min}$, the mixture was allowed to warm to $0{ }^{\circ} \mathrm{C}$, and additional reagents [imidazole $(22 \mathrm{mg}, 0.66$ $\mathrm{mmol})$ and $\left.\mathrm{I}_{2}(16 \mathrm{mg}, 0.06 \mathrm{mmol})\right]$ were introduced. After being stirred at $0{ }^{\circ} \mathrm{C}$ for $20 \mathrm{~h}$, the reaction mixture was quenched with saturated aqueous $\mathrm{NH}_{4} \mathrm{Cl}$, and extracted with hexane/EtOAc (x2). The organic layer was washed with brine (x2), and dried over $\mathrm{MgSO}_{4}$. Concentration and flash column chromatography (hexane/EtOAc 1:0-10:1) gave the iodide (460 mg, $0.58 \mathrm{mmol})$ in $87 \%$ yield: colorless oil; $[\alpha]_{\mathrm{D}}{ }^{20}=19.5\left(c 1.00, \mathrm{CHCl}_{3}\right) ;$ IR (film) v 3432, 2943, 1462, 1111, 1061, $824 \mathrm{~cm}^{-1} ;{ }^{1} \mathrm{H}$ NMR (500 $\left.\mathrm{MHz}, \mathrm{CDCl}_{3}\right) \delta$ 1.02-1.05 (33H, m, TIPS, TBPS, Me53), 1.09 (3H, d, J=7.0 Hz, Me54), 1.37 (1H, d, $J=$ $5.5 \mathrm{~Hz}, \mathrm{OH} 38), 1.42-1.50$ (2H, m, H35, H37), 1.71 (1H, m, H35), 1.71 (1H, ddd, $J=12.0,12.0,11.5$ Hz, H32), 1.80-1.94 (2H, m, H36, H37), 2.69 (1H, ddd, $J=12.0,4.5,4.5 \mathrm{~Hz}, \mathrm{H} 32), 3.10$ (1H, dd, $J=$ 10.0, 8.5 Hz, H40), 3.19 (1H, ddd, $J=11.5,9.5,4.5 \mathrm{~Hz}, \mathrm{H} 33), 3.36$ (1H, ddd, $J=9.5,9.5,3.0 \mathrm{~Hz}, \mathrm{H} 34)$, 3.43-3.52 (2H, m, H38, 39), 3.56 (1H, d, J = 10.5 Hz, H29), 3.68 (1H, d, J = 10.5 Hz, H29), $3.71(1 \mathrm{H}$, $\mathrm{dd}, J=10.0,2.0 \mathrm{~Hz}, \mathrm{H} 40), 4.08(1 \mathrm{H}, \mathrm{dd}, J=12.0,4.5 \mathrm{~Hz}, \mathrm{H} 31), 7.31-7.40$ (6H, m, TBPS), 7.71-7.74 (4H, m, TBPS); ${ }^{13} \mathrm{C}$ NMR (125 MHz, $\left.\mathrm{CDCl}_{3}\right) \delta 10.6,12.4,12.7,18.26,18.27,19.3,26.8,27.5,27.9$, $37.6,45.2,47.3,67.6,68.5,73.0,75.3,77.8,83.6,88.2,127.2,127.3,129.2,129.3,133.8,134.2,135.7$, 
136.0; HRMS (MALDI) calcd for $\mathrm{C}_{39} \mathrm{H}_{63} \mathrm{IO}_{5} \mathrm{Si}_{2} \mathrm{Na}$ 817.3156 $\left(\mathrm{M}+\mathrm{Na}^{+}\right)$, found 817.3141.

To a solution of the iodide $(671 \mathrm{mg}, 843 \mu \mathrm{mol})$ in DMSO $(1.7 \mathrm{~mL})$ at room temperature was added $\mathrm{NaCN}(83 \mathrm{mg}, 1.69 \mathrm{mmol})$. The mixture was stirred at $40{ }^{\circ} \mathrm{C}$ for $2 \mathrm{~d}$. The reaction mixture was quenched with $\mathrm{H}_{2} \mathrm{O}$, and extracted with EtOAc (x2). The organic layer was washed with $\mathrm{H}_{2} \mathrm{O}$, and dried over $\mathrm{MgSO}_{4}$. Concentration and flash column chromatography (hexane/EtOAc 1:0-6:1) gave nitrile 35 (547 mg, $842 \mu \mathrm{mol})$ in 97\% yield: colorless oil; $[\alpha]_{\mathrm{D}}{ }^{20}=5.46\left(c 1.51, \mathrm{CHCl}_{3}\right)$; IR (film) $v 3488$, 2943, 2360, 1462, 1427, 1106, $882 \mathrm{~cm}^{-1} ;{ }^{1} \mathrm{H}$ NMR (500 MHz, $\left.\mathrm{CDCl}_{3}\right) \delta$ 1.02-1.06 (33H, m, TIPS, TBPS, Me53), $1.10(3 \mathrm{H}, \mathrm{d}, J=7.0 \mathrm{~Hz}, \mathrm{Me} 54), 1.26(1 \mathrm{H}, \mathrm{d}, J=5.0 \mathrm{~Hz}, \mathrm{OH} 38), 1.46(1 \mathrm{H}, \mathrm{m}, \mathrm{H} 35), 1.67$ (1H, m, H37), $1.74(1 \mathrm{H}, \mathrm{ddd}, J=11.5,11.5,11.5 \mathrm{~Hz}, \mathrm{H} 32), 1.82-1.92(3 \mathrm{H}, \mathrm{m}, \mathrm{H} 35, \mathrm{H} 36, \mathrm{H} 37)$, 2.44-2.49 (2H, m, H32, H40), 2.89 (1H, dd, $J=16.5,3.0 \mathrm{~Hz}, \mathrm{H} 40), 3.20(1 \mathrm{H}, \mathrm{ddd}, J=11.5,11.5,5.0$ Hz, H33), 3.35 (1H, ddd, $J=11.5,11.5,3.0 \mathrm{~Hz}, \mathrm{H} 34), 3.48(1 \mathrm{H}, \mathrm{ddd}, J=10.0,10.0,2.5 \mathrm{~Hz}, \mathrm{H} 38)$, 3.54-3.58 (2H, m, H29, H39), 3.69 (1H, d, $J=10.5 \mathrm{~Hz}, \mathrm{H} 29), 4.10(1 \mathrm{H}, \mathrm{dd}, J=11.5,5.0 \mathrm{~Hz}, \mathrm{H} 31)$, 7.32-7.42 (6H, m, TBPS), 7.72-7.75 (4H, m, TBPS); $\left.{ }^{13} \mathrm{C} \mathrm{NMR} \mathrm{(125} \mathrm{MHz,} \mathrm{CDCl}_{3}\right) \delta 12.4,12.6,18.20$, $18.22,19.3,23.9,26.8,27.7,27.9,37.0,45.6,48.2,67.3,68.4,73.0,74.1,77.8,84.2,84.4,118.6,127.2$, 127.3, 129.2, 129.3, 133.8, 134.2, 135.7, 136.0; MALDI-TOF MS, calcd for $\mathrm{C}_{40} \mathrm{H}_{63} \mathrm{O}_{5} \mathrm{Si}_{2} \mathrm{Na}_{716.41}$ $\left(\mathrm{M}+\mathrm{Na}^{+}\right)$, found 716.42; Anal. calcd for $\mathrm{C}_{40} \mathrm{H}_{63} \mathrm{O}_{5} \mathrm{Si}_{2}: \mathrm{C}, 69.22, \mathrm{H}, 9.15, \mathrm{~N}, 2.02$, found $\mathrm{C}, 69.01, \mathrm{H}, 9.23$, $\mathrm{N}, 1.80$.

TES ether 36. To a solution of nitrile $35(201 \mathrm{mg}, 0.24 \mathrm{mmol})$ and 2,6-lutidine $(42 \mu \mathrm{l}, 0.36 \mathrm{mmol})$ in $\mathrm{CH}_{2} \mathrm{Cl}_{2}(0.5 \mathrm{~mL})$ at $-78{ }^{\circ} \mathrm{C}$ was added TESOTf $(59 \mu \mathrm{l}, 0.26 \mathrm{mmol})$. After being stirred at $-78^{\circ} \mathrm{C}$ for 1 h, the reaction mixture was quenched with $\mathrm{H}_{2} \mathrm{O}$, and extracted with hexane/EtOAc (x2). The organic 
layer was washed with $\mathrm{H}_{2} \mathrm{O}$ and brine, and dried over $\mathrm{MgSO}_{4}$. Concentration and flash column chromatography (hexane/EtOAc 1:0-50:1) gave TES ether 36 (201 mg, $0.24 \mathrm{mmol}$ ) in 100\% yield: colorless oil; $[\alpha]_{\mathrm{D}}{ }^{21}=10.1\left(c 1.16, \mathrm{CHCl}_{3}\right)$; IR (film) $v 2955,2361,1461,1106,819,741 \mathrm{~cm}^{-1} ;{ }^{1} \mathrm{H}$ NMR (500 MHz, $\left.\mathrm{CDCl}_{3}\right) \delta 0.59$ (6H, m, TES), 0.95 (9H, t, $\left.J=8.5 \mathrm{~Hz}, \mathrm{TES}\right), 1.01-1.07$ (36H, m, TIPS, TBPS, Me53, Me54), 1.48 (1H, m, H35), 1.59-1.75 (2H, m, H32, H37), 1.78-1.88 (3H, m, H35, H36, H37), $2.35(1 \mathrm{H}, \mathrm{dd}, J=16.5,8.5 \mathrm{~Hz}, \mathrm{H} 40), 2.46(1 \mathrm{H}, \mathrm{ddd}, J=11.5,5.0,5.0 \mathrm{~Hz}, \mathrm{H} 32), 2.80(1 \mathrm{H}, \mathrm{dd}, J=16.5$, $3.5 \mathrm{~Hz}, \mathrm{H} 40), 3.21(1 \mathrm{H}, \mathrm{ddd}, J=11.5,10.0,5.0 \mathrm{~Hz}, \mathrm{H} 33), 3.34$ (1H, ddd, $J=10.0,10.0,3.5 \mathrm{~Hz}, \mathrm{H} 34)$, $3.46(1 \mathrm{H}, \mathrm{ddd}, J=10.0,10.0,3.0 \mathrm{~Hz}, \mathrm{H} 38), 3.54-3.58(2 \mathrm{H}, \mathrm{m}, \mathrm{H} 29, \mathrm{H} 39), 3.68(1 \mathrm{H}, \mathrm{d}, J=10.5 \mathrm{~Hz}$, H29), $4.08(1 \mathrm{H}, \mathrm{dd}, J=11.5,5.0 \mathrm{~Hz}, \mathrm{H} 31), 7.31-7.40(6 \mathrm{H}, \mathrm{m}, \mathrm{TBPS}), 7.71-7.74(4 \mathrm{H}, \mathrm{m}, \mathrm{TBPS}) ;{ }^{13} \mathrm{C}$ NMR (125 MHz, $\left.\mathrm{CDCl}_{3}\right) \delta 4.9,6.8,12.4,12.6,18.20,18.22,19.3,23.8,26.8,26.9,27.6,28.0,37.0$, $45.6,47.7,67.3,68.4,72.9,75.1,77.8,84.1,84.9,118.6,127.2,127.3,129.2,129.3,133.8,134.2,135.7$, 136.0; MALDI-TOF MS, calcd for $\mathrm{C}_{46} \mathrm{H}_{77} \mathrm{NO}_{5} \mathrm{Si}_{3} \mathrm{Na} 830.50\left(\mathrm{M}+\mathrm{Na}^{+}\right)$, found 830.50; Anal. calcd for $\mathrm{C}_{46} \mathrm{H}_{77} \mathrm{NO}_{5} \mathrm{Si}_{3}: \mathrm{C}, 68.35, \mathrm{H}, 9.60, \mathrm{~N}, 1.73$, found $\mathrm{C}, 68.11, \mathrm{H}, 9.77, \mathrm{~N}, 1.67$.

Dithioacetal 37. To a solution of TES ether $36(254 \mathrm{mg}, 0.31 \mathrm{mmol})$ in $\mathrm{CH}_{2} \mathrm{Cl}_{2}(1.0 \mathrm{~mL})$ at $-78{ }^{\circ} \mathrm{C}$ was added DIBAL (0.95 $\mathrm{M}$ in hexane, $1.0 \mathrm{~mL}, 0.95 \mathrm{mmol})$ over $10 \mathrm{~min}$. After being stirred at $-78^{\circ} \mathrm{C}$ for $50 \mathrm{~min}$, the reaction mixture was quenched with EtOAc, and stirred with saturated aqueous Rochelle's salt at room temperature for $2 \mathrm{~h}$. The mixture was extracted with EtOAc (x3), and the organic layer was washed with brine and dried over $\mathrm{MgSO}_{4}$. Concentration and purification through a pad of florisil gave the aldehyde, which was used in the next reaction without further purification.

To the aldehyde and $\mathrm{PhSSPh}(338 \mathrm{mg}, 1.55 \mathrm{mmol})$ at room temperature was added $\mathrm{Bu}_{3} \mathrm{P}(0.46 \mathrm{~mL}, 1.86$ 
mmol). The mixture was stirred at $40{ }^{\circ} \mathrm{C}$ for $1 \mathrm{~d}$, and then exposed to air over night. The solution was directly subjected to alumina column chromatography (hexane/EtOAc 1:0-100:1) to give dithioacetal $37(260 \mathrm{mg}, 0.25 \mathrm{mmol})$ in $83 \%$ yield over 2 steps: colorless oil; $[\alpha]_{\mathrm{D}}{ }^{20}=-53.7(c 1.11$, $\mathrm{CHCl}_{3}$ ); IR (film) v 3071, 2952, 2867, 1463, 1098, 1009, $819 \mathrm{~cm}^{-1} ;{ }^{1} \mathrm{H} \mathrm{NMR}\left(500 \mathrm{MHz}, \mathrm{CDCl}_{3}\right) \delta 0.50$, (6H, m, TES), 0.88 (9H, t, $J=8.0 \mathrm{~Hz}, \mathrm{TES}) .0 .98$ (21H, s, TIPS), 1.03 (9H, s, TBPS), 1.05 (3H, s, Me53), 1.06 (3H, d, $J=7.0 \mathrm{~Hz}, \mathrm{Me} 54), 1.56-1.87$ (7H, m, H32, H35x2, H36, H37x2, H40), 2.17 (1H, ddd, $J=14.0,11.5,2.0 \mathrm{~Hz}, \mathrm{H} 40), 2.26(1 \mathrm{H}, \mathrm{ddd}, J=12.0,5.0,5.0 \mathrm{~Hz}, \mathrm{H} 32), 3.34-3.45$ (3H, m, H33, H34, H38), 3.56 (1H, d, $J=10.5 \mathrm{~Hz}, \mathrm{H} 29), 3.66(1 \mathrm{H}, \mathrm{d}, J=10.5 \mathrm{~Hz}, \mathrm{H} 29), 3.83(1 \mathrm{H}, \mathrm{ddd}, J=10.0$, 10.0, $2.0 \mathrm{~Hz}, \mathrm{H} 39), 4.04(1 \mathrm{H}, \mathrm{dd}, J=12.0,5.0 \mathrm{~Hz}, \mathrm{H} 31), 4.60(1 \mathrm{H}, \mathrm{dd}, J=11.5,3.0 \mathrm{~Hz}, \mathrm{H} 41)$, 7.24-7.76 (20H, m, TBPS, PhSx2); ${ }^{13} \mathrm{C}$ NMR (125 MHz, $\left.\mathrm{CDCl}_{3}\right)$ ઈ 5.2, 5.3, 7.7, 12.6, 12.9, 18.48, 18.49, $19.6,27.1,27.3,28.0,37.6,40.6,45.2,45.7,54.0,67.7,68.8,73.1,76.3,77.8,82.0,84.4,127.5,127.6$, 128.3, 128.9, 129.1, 129.5, 131.7, 132.3, 134.0, 134.6, 134.8, 135.9, 136.3; MALDI-TOF MS, calcd for $\mathrm{C}_{58} \mathrm{H}_{88} \mathrm{O}_{5} \mathrm{~S}_{2} \mathrm{Si}_{3} \mathrm{Na} 1035.52\left(\mathrm{M}+\mathrm{Na}^{+}\right)$, found 1035.53; Anal. calcd for $\mathrm{C}_{58} \mathrm{H}_{88} \mathrm{O}_{5} \mathrm{~S}_{2} \mathrm{Si}_{3}: \mathrm{C}, 68.72, \mathrm{H}, 8.75$, found C, 68.48, H, 9.01.

HI ring segment 9. To a solution of TES ether 37 (443 mg, $437 \mu \mathrm{mol})$ in $\mathrm{MeOH} / \mathrm{CH}_{2} \mathrm{Cl}_{2}(5: 1,9 \mathrm{~mL})$ at $0{ }^{\circ} \mathrm{C}$ was added PPTS $(110 \mathrm{mg}, 437 \mu \mathrm{mol})$. After being stirred at $0{ }^{\circ} \mathrm{C}$ for $12 \mathrm{~h}$, the mixture was quenched with saturated aqueous $\mathrm{NaHCO}_{3}$, and extracted with EtOAc (x2). The organic layer was washed with brine, and dried over $\mathrm{MgSO}_{4}$. Concentration and flash column chromatography (hexane/EtOAc 30:1-0:1) gave HI ring segment 9 (373 mg, $415 \mu \mathrm{mol})$ in 94\% yield: colorless oil; [ $\alpha]_{\mathrm{D}}{ }^{21}$ $=-76.8\left(c\right.$ 1.33, $\left.\mathrm{CHCl}_{3}\right)$; IR (film) v 3464, 2941, 1464, 1100, 820, $739 \mathrm{~cm}^{-1} ;{ }^{1} \mathrm{H} \mathrm{NMR}(500 \mathrm{MHz}$, 
$\left.\mathrm{CDCl}_{3}\right) \delta 0.99(21 \mathrm{H}, \mathrm{s}, \mathrm{TIPS}), 1.04$ (9H, s, TBPS), 1.05 (3H, s, Me53), 1.09 (3H, d, J = 7.0 Hz, Me54), 1.56 (1H, m, H35), 1.64-1.74 (3H, m, H32, H35, H37), 1.83-1.97 (3H, m, H36, H37, H40), 2.21-2.27 (2H, m, H32, H40), 3.30-3.38 (2H, m, H33, H34), 3.44 (1H, ddd, J = 9.5, 9.5, 3.0 Hz, H38), 3.57 (1H, d, $J=10.0 \mathrm{~Hz}, \mathrm{H} 29), 3.67(1 \mathrm{H}, \mathrm{d}, J=10.0 \mathrm{~Hz}, \mathrm{H} 29), 3,79(1 \mathrm{H}, \mathrm{ddd}, J=9.5,9.5,2.5 \mathrm{~Hz}, \mathrm{H} 39), 4.04(1 \mathrm{H}$, $\mathrm{dd}, J=12.5,5.0 \mathrm{~Hz}, \mathrm{H} 31), 4.62(1 \mathrm{H}, \mathrm{dd}, J=11.5,3.5 \mathrm{~Hz}, \mathrm{H} 41), 7.27-7.75(20 \mathrm{H}, \mathrm{m}, \mathrm{PhSx} 2, \mathrm{TBPS}) ;{ }^{13} \mathrm{C}$ NMR (125 MHz, $\left.\mathrm{CDCl}_{3}\right) \delta 12.3,12.7,18.21,18.23,19.3,26.8,27.2,27.8,37.4,40.5,45.0,45.6,53.7$, $67.4,68.6,72.8,75.2,77.6,82.2,83.9,127.2,127.3,127.4,128.2,128.7,128.9,129.3,131.6,131.8$, 133.8, 134.1, 134.2, 134.7, 135.7, 136.0; MALDI-TOF MS, calcd for $\mathrm{C}_{52} \mathrm{H}_{74} \mathrm{O}_{5} \mathrm{~S}_{2} \mathrm{Si}_{2} \mathrm{Na}\left(\mathrm{M}+\mathrm{Na}^{+}\right) 921.44$ found 921.44; Anal. calcd for $\mathrm{C}_{52} \mathrm{H}_{74} \mathrm{O}_{5} \mathrm{~S}_{2} \mathrm{Si}_{2}: \mathrm{C}, 69.44, \mathrm{H}, 8.29$, found $\mathrm{C}, 69.60, \mathrm{H}, 8.51$.

Ester 38. To a mixture of alcohol 9 (144 mg, $160 \mu \mathrm{mol})$ and carboxylic acid 10 (79.3 mg, $168 \mu \mathrm{mol})$ in toluene $(8 \mathrm{~mL})$ at room temperature were added $\mathrm{Et}_{3} \mathrm{~N}(134 \mu \mathrm{l}, 960 \mu \mathrm{mol}), 2,4,6$-trichlorobenzoyl chloride $(125 \mu \mathrm{l}, 800 \mu \mathrm{mol})$ and DMAP $(195 \mathrm{mg}, 1600 \mu \mathrm{mol})$. After being stirred at $40{ }^{\circ} \mathrm{C}$ for $5.5 \mathrm{~h}$, the reaction mixture was quenched with saturated aqueous $\mathrm{NH}_{4} \mathrm{Cl}$, and extracted with hexane/EtOAc (x2). The organic layer was washed with brine, and dried over $\mathrm{MgSO}_{4}$. Concentration and flash column chromatography (hexane/EtOAc 1:0-20:1) gave ester 38 (195 mg, $144 \mu \mathrm{mol})$ in 90\% yield: colorless oil; $[\alpha]_{\mathrm{D}}{ }^{27}=-74.2\left(c 0.65\right.$, benzene); IR (film) $v 2941,1732,1462,1100,1030,703 \mathrm{~cm}^{-1} ;{ }^{1} \mathrm{H}$ NMR (500 MHz, CDCl $) \delta 0.92(3 \mathrm{H}, \mathrm{d}, J=6.5 \mathrm{~Hz}, \mathrm{Me} 57), 0.99-1.02(30 \mathrm{H}, \mathrm{m}, \mathrm{TIPS}, \mathrm{TBPS}), 1.03(3 \mathrm{H}$, $\mathrm{d}, J=7.0 \mathrm{~Hz}, \mathrm{Me} 56), 1.05(3 \mathrm{H}, \mathrm{s}, \mathrm{Me} 53), 1.07(3 \mathrm{H}, \mathrm{d}, J=7.5 \mathrm{~Hz}, \mathrm{Me} 54), 1.24(3 \mathrm{H}, \mathrm{d}, J=7.0 \mathrm{~Hz}$, Me55), $1.56(1 \mathrm{H}, \mathrm{dq}, J=11.5,6.5 \mathrm{~Hz}, \mathrm{H} 48), 1.60(1 \mathrm{H}, \mathrm{m}, \mathrm{H} 35), 1.68(1 \mathrm{H}, \mathrm{ddd}, J=11.5,11.5,11.5 \mathrm{~Hz}$, H32), 1.69-1.81 (4H, m, H37, H47, H50, H51), 1.83-2.01 (6H, m, H35, H36, H37, H40, H50, H51), 
$2.01(1 \mathrm{H}, \mathrm{ddd}, J=12.0,9.0,2.0 \mathrm{~Hz}, \mathrm{H} 40), 2.22(1 \mathrm{H}, \mathrm{ddd}, J=11.5,4.5,4.5 \mathrm{~Hz}, \mathrm{H} 32), 2.80(1 \mathrm{H}, \mathrm{dq}, J=$ 7.0, 7.0 Hz, H43), 3.07 (1H, dd, $J=10.0,10.0 \mathrm{~Hz}, \mathrm{H} 46), 3.37$ (3H, s, MOM), 3.29-3.41 (2H, m, H33, H34), 3.57 (1H, d, $J=10.0$ Hz, H29), 3.67 (1H, d, $J=10.0 \mathrm{~Hz}, \mathrm{H} 29), 3.82-3.91$ (2H, m, H52x2), 3.94 $(1 \mathrm{H}, \mathrm{d}, J=7.0 \mathrm{~Hz}, \mathrm{H} 44), 3.95(1 \mathrm{H}, \mathrm{d}, J=10.0 \mathrm{~Hz}, \mathrm{H} 45), 4.03(1 \mathrm{H}, \mathrm{dd}, J=11.5,4.5 \mathrm{~Hz}, \mathrm{H} 31), 4.11(1 \mathrm{H}$, dd, $J=9.0,9.0 \mathrm{~Hz}, \mathrm{H} 39), 4.58(1 \mathrm{H}, \mathrm{dd}, J=11.0 \mathrm{~Hz}, 2.0 \mathrm{~Hz}, \mathrm{H} 41), 4.61(1 \mathrm{H}, \mathrm{d}, J=6.5 \mathrm{~Hz}, \mathrm{MOM}), 4.63$ $(1 \mathrm{H}, \mathrm{ddd}, J=9.0,9.0,3.0 \mathrm{~Hz}, \mathrm{H} 38), 4.69(1 \mathrm{H}, \mathrm{d}, J=12.0 \mathrm{~Hz}, \mathrm{NAP}), 4.76(1 \mathrm{H}, \mathrm{d}, J=6.5 \mathrm{~Hz}, \mathrm{MOM})$ $4.88(1 \mathrm{H}, \mathrm{d}, J=12.0 \mathrm{~Hz}, \mathrm{NAP}), 7.22-7.59$ (19H, m, NAP, TBPS, PhSx2), 7.70-7.85 (8H, m, NAP, TBPS $) ;{ }^{13} \mathrm{C}$ NMR $\left(125 \mathrm{MHz}, \mathrm{CDCl}_{3}\right) \delta 11.0,12.5,12.7,13.6,14.2,15.8,18.22,18.23,19.4,23.0,23.7$, $24.4,26.3,26.9,28.2,28.9,29.7,30.3,35.1,37.3,38.7,39.5,39.9,40.2,41.4,42.1,44.3,53.7,56.5$, $67.48,67.51,68.1,68.6,72.6,72.9,73.6,76.4,77.7,79.6,80.6,81.0,82.4,98.5,107.9,125.6,125.9$, $126.0,126.3,127.3,127.4,127.7,127.8,127.9,128.1,128.8,128.9,129.3,130.9,131.7,132.0,132.9$, 133.3, 133.8, 134.3, 134.9, 135.7, 136.1, 136.4, 174.6; HRMS (FAB), calcd for $\mathrm{C}_{79} \mathrm{H}_{108} \mathrm{O}_{11} \mathrm{~S}_{2} \mathrm{Si}_{2} \mathrm{Na}$ 1357.6769 $\left(\mathrm{M}+\mathrm{Na}^{+}\right)$, found 1375.6763 .

Cyclic enol ether 39. Powdered MS4A (50 mg), and $\mathrm{Cp}_{2} \mathrm{TiCl}_{2}(108 \mathrm{mg}, 432 \mu \mathrm{mol})$ were placed in a flask and dried with a heat gun under reduced pressure. $\mathrm{Mg}$ turning (13 mg, $540 \mu \mathrm{mol})$, THF (3 mL) and $\mathrm{P}(\mathrm{OEt})_{3}(150 \mu \mathrm{l}, 864 \mu \mathrm{mol})$ were successively added to this flask at room temperature, and the reaction mixture was stirred for $3 \mathrm{~h}$. Then, to this mixture was added ester 38 (73 $\mathrm{mg}, 54 \mu \mathrm{mol})$ in THF ( $2 \mathrm{~mL})$ dropwise at room temperature. After being heated to reflux for $1 \mathrm{~h}$, the reaction mixture was quenched with $1 \mathrm{M} \mathrm{NaOH}(4 \mathrm{~mL})$ at room temperature, and the resulting insoluble solid was filtered off. The mixture was extracted with $\mathrm{Et}_{2} \mathrm{O}(\mathrm{x} 2)$, and the organic layer was washed with brine. 
The violet organic layer was dried over $\mathrm{MgSO}_{4}$ overnight to give a colorless solution. Concentration and flash column chromatography (deactivated with $1 \% \mathrm{Et}_{3} \mathrm{~N} /$ hexane, hexane/EtOAc 1:0-15:1) gave cyclic enol ether 39 (48 mg, $43 \mu \mathrm{mol})$ in $80 \%$ yield: colorless oil; $[\alpha]_{\mathrm{D}}{ }^{21}=-17.7\left(c 0.98, \mathrm{CHCl}_{3}\right)$; IR (film) v 2932, 2867, 1675, 1587, 1463, $1105 \mathrm{~cm}^{-1} ;{ }^{1} \mathrm{H}$ NMR $\left(500 \mathrm{MHz}, \mathrm{CDCl}_{3}\right) \delta 0.93(3 \mathrm{H}, \mathrm{d}, J=6.5$ Hz, Me57), 1.04-1.09 (39H, m, Me53, Me54, Me56, TBPS, TIPS), 1.17 (3H, d, $J=7.0 \mathrm{~Hz}, \mathrm{Me} 55$ ), 1.49-1.57 (2H, m, H35, H48), 1.64-1.76 (3H, m, H32, H37, H47), 1.76-1.85 (2H, m, H50, H51), 1.86-1.99 (4H, m, H35, H36, H37, H50), 2.03 (1H, m, H51), 2.09 (1H, ddd, $J=16.5,9.5,2.0$ Hz, H40), $2.15(1 \mathrm{H}, \mathrm{ddd}, J=12.0,4.5,4.5 \mathrm{~Hz}, \mathrm{H} 32), 2.23(1 \mathrm{H}, \mathrm{ddd}, J=16.5,5.5,5.5 \mathrm{~Hz}, \mathrm{H} 40), 2.50(1 \mathrm{H}, \mathrm{dq}, J=$ 9.5, 7.0 Hz, H43), $3.21(1 \mathrm{H}, \mathrm{ddd}, J=9.5,9.5,4.5 \mathrm{~Hz}, \mathrm{H} 33), 3.23(1 \mathrm{H}, \mathrm{dd}, J=10.0,10.0 \mathrm{~Hz}, \mathrm{H} 46)$, 3.38-3.44 (2H, m, H34, H39), 3.41 (3H, s, MOM), 3.51 (1H, ddd, $J=10.0,10.0,2.5 \mathrm{~Hz}, \mathrm{H} 38), 3.58$ $(1 \mathrm{H}, \mathrm{d}, J=10.0 \mathrm{~Hz}, \mathrm{H} 29), 3.60(1 \mathrm{H}, \mathrm{d}, J=9.5 \mathrm{~Hz}, \mathrm{H} 44), 3.70(1 \mathrm{H}, \mathrm{d}, J=10.0 \mathrm{~Hz}, \mathrm{H} 29), 3.79$ (1H, ddd, $J=7.5,7.5,7.5 \mathrm{~Hz}, \mathrm{H} 52), 3.90(1 \mathrm{H}, \mathrm{ddd}, J=7.5,7.5,4.5 \mathrm{~Hz}, \mathrm{H} 52), 3.96(1 \mathrm{H}, \mathrm{d}, J=10.0 \mathrm{~Hz}, \mathrm{H} 45)$, $4.09(1 \mathrm{H}, \mathrm{dd}, J=12.0,4.5 \mathrm{~Hz}, \mathrm{H} 31), 4.48(1 \mathrm{H}, \mathrm{dd}, J=5.5,2.0 \mathrm{~Hz}, \mathrm{H} 41), 4.64(1 \mathrm{H}, \mathrm{d}, J=6.5 \mathrm{~Hz}$, MOM), $4.68(1 \mathrm{H}, \mathrm{d}, J=12.0 \mathrm{~Hz}, \mathrm{NAP}), 4.89(1 \mathrm{H}, \mathrm{d}, J=6.5 \mathrm{~Hz}, \mathrm{MOM}), 4.94(1 \mathrm{H}, \mathrm{d}, J=12.0 \mathrm{~Hz}$, NAP), 7.30-7.57 (9H, m, NAP, TBPS), 7.70-7.86 (8H, m, NAP, TBPS); ${ }^{13} \mathrm{C}$ NMR (125 MHz, $\mathrm{CDCl}_{3}$ ) $\delta 10.9,12.4,12.8,13.6,14.0,15.9,16.3,18.21,18.22,19.4,23.0,23.7,24.4,26.9,27.3,28.9,30.3,30.8$, 31.6, 35.0, 37.9, 38.7, 39.8, 40.8, 42.2, 46.3, 56.4, 67.1, 67.3, 68.1, 68.5, 72.7, 73.4, 77.6, 79.1, 80.1, $82.5,82.7,94.5,98.4,107.9,125.6,125.9,126.2,126.3,127.3,127.4,127.6,127.7,127.8,128.8,129.3$, $130.9,132.8,133.2,133.8,134.3,135.7,136.1,136.7,155.9$; HRMS (FAB), calcd for $\mathrm{C}_{67} \mathrm{H}_{98} \mathrm{O}_{10} \mathrm{Si}_{2} \mathrm{Na}$ $1141.6596\left(\mathrm{M}+\mathrm{Na}^{+}\right)$, found 1141.6593 . 
Ketone 42 $\beta$. To a solution of cyclic enol ether $39(41.5 \mathrm{mg}, 37.1 \mu \mathrm{mol})$ in $\mathrm{THF}(0.4 \mathrm{~mL})$ at $0{ }^{\circ} \mathrm{C}$ was added $\mathrm{BH}_{3} \cdot \mathrm{SMe}_{2}(2.0 \mathrm{M}$ in THF, $25 \mathrm{~mL}, 50 \mu \mathrm{mol})$. After $2.5 \mathrm{~h}$ at room temperature, $15 \%$ aqueous $\mathrm{NaOH}(50 \mu \mathrm{l}, 220 \mu \mathrm{mol})$ was added to the reaction mixture at $0{ }^{\circ} \mathrm{C}$, followed by addition of $30 \%$ aqueous $\mathrm{H}_{2} \mathrm{O}_{2}(50 \mu \mathrm{l}, 440 \mu \mathrm{mol})$. After being stirred overnight at room temperature, the mixture was extracted with EtOAc (x2), and the organic layer was washed with brine, and dried over $\mathrm{MgSO}_{4}$. Concentration and flash column chromatography (hexane/EtOAc 10:1-3:1) gave a 3:1 inseparable mixture of 40 and $\mathbf{4 1}(32.1 \mathrm{mg}, 28.2 \mu \mathrm{mol})$ in $76 \%$ combined yield.

To a solution of the alcohols 40 and $41(32.1 \mathrm{mg}, 28.2 \mu \mathrm{mol})$ in $\mathrm{CH}_{2} \mathrm{Cl}_{2}(3 \mathrm{~mL})$ at $0{ }^{\circ} \mathrm{C}$ was added DessMartin periodinane $(35 \mathrm{mg}, 84 \mu \mathrm{mol})$. After being stirred at room temperature for $3 \mathrm{~h}$, the reaction mixture was quenched with aqueous $\mathrm{Na}_{2} \mathrm{~S}_{2} \mathrm{O}_{3} / \mathrm{NaHCO}_{3}$. The organic layer was washed with brine, dried over $\mathrm{MgSO}_{4}$, and concentrated. The residue was purified through a pad of silica gel, and the resulting ketones were separated by HPLC (YMC-Pack SIL-06, 10x250 mm, UV $254 \mathrm{~nm}$, hexane/EtOAc 17:1, $5.0 \mathrm{~mL} / \mathrm{min})$ to give ketone $\mathbf{4 2} \beta\left(\mathrm{t}_{\mathrm{R}}=21.3 \mathrm{~min}, 7.7 \mathrm{mg}, 6.7 \mu \mathrm{mol}, 24 \%\right)$ and its C42epimer $\mathbf{4 2} \alpha\left(\mathrm{t}_{\mathrm{R}}=17.9 \mathrm{~min}, 22.7 \mathrm{mg}, 20.0 \mu \mathrm{mol}, 71 \%\right) . \quad \mathbf{4 2} \alpha$ : colorless oil; $[\alpha]_{\mathrm{D}}^{20}=-49.2(c$ 1.00, $\left.\mathrm{CHCl}_{3}\right) ; \mathrm{IR}(\mathrm{film}) \vee 2942,2867,1723,1461,1104,820 \mathrm{~cm}^{-1} ;{ }^{1} \mathrm{H}$ NMR $\left(500 \mathrm{MHz}, \mathrm{CDCl}_{3}\right) \delta 0.93(3 \mathrm{H}, \mathrm{d}$, $J=7.0 \mathrm{~Hz}, \mathrm{Me} 57), 0.94(3 \mathrm{H}, \mathrm{d}, J=7.0 \mathrm{~Hz}, \mathrm{Me} 55), 1.02-1.05$ (33H, m, TIPS, TBPS, Me53), 1.06 (3H, $\mathrm{d}, J=7.5 \mathrm{~Hz}, \mathrm{Me} 54), 1.08(3 \mathrm{H}, \mathrm{d}, J=6.5 \mathrm{~Hz}, \mathrm{Me} 56), 1.42-1.58(3 \mathrm{H}, \mathrm{m}, \mathrm{H} 35, \mathrm{H} 37, \mathrm{H} 48), 1.71(1 \mathrm{H}, \mathrm{ddd}$, $J=12.0,12.0,12.0 \mathrm{~Hz}, \mathrm{H} 32), 1.75-2.06(8 \mathrm{H}, \mathrm{m}, \mathrm{H} 35, \mathrm{H} 36, \mathrm{H} 37, \mathrm{H} 47, \mathrm{H} 50 x 2, \mathrm{H} 51 \times 2), 2.09$ (1H, ddd, $J=12.0,5.0,5.0 \mathrm{~Hz}, \mathrm{H} 32), 2.47(1 \mathrm{H}, \mathrm{dqd}, J=10.0,7.0,3.0 \mathrm{~Hz}, \mathrm{H} 43), 2.61(1 \mathrm{H}, \mathrm{dd}, J=14.0,11.5 \mathrm{~Hz}$, H40), $2.75(1 \mathrm{H}, \mathrm{dd}, J=14.0,5.0 \mathrm{~Hz}, \mathrm{H} 40), 3.11(1 \mathrm{H}, \mathrm{ddd}, J=12.0,9.0,5.0 \mathrm{~Hz}, \mathrm{H} 33), 3.15(1 \mathrm{H}, \mathrm{dd}, J=$ 10.0, $10.0 \mathrm{~Hz}, \mathrm{H} 46), 3.31$ (1H, ddd, $J=9.0,9.0,2.5 \mathrm{~Hz}, \mathrm{H} 34), 3.36$ (3H, s, MOM), 3.53 (1H, ddd, $J=$ 
11.5, 9.0, 5.0 Hz, H39), 3.55 (1H, d, $J=10.5 \mathrm{~Hz}, \mathrm{H} 29), 3.68$ (1H, d, $J=10.5 \mathrm{~Hz}, \mathrm{H} 29), 3.70$ (1H, ddd, $J$ $=9.0,9.0,2.5 \mathrm{~Hz}, \mathrm{H} 38), 3.80(1 \mathrm{H}, \mathrm{d}, J=10.0 \mathrm{~Hz}, \mathrm{H} 42), 3.84-3.92(3 \mathrm{H}, \mathrm{m}, \mathrm{H} 44, \mathrm{H} 52 \times 2), 4.08(1 \mathrm{H}, \mathrm{dd}$, $J=12.0,5.0 \mathrm{~Hz}, \mathrm{H} 31), 4.19(1 \mathrm{H}, \mathrm{dd}, J=10.0,2.5 \mathrm{~Hz}, \mathrm{H} 45), 4.64(1 \mathrm{H}, \mathrm{d}, J=7.0 \mathrm{~Hz}, \mathrm{MOM}), 4.65(1 \mathrm{H}$, $\mathrm{d}, J=7.0 \mathrm{~Hz}, \mathrm{MOM}), 4.67(1 \mathrm{H}, \mathrm{d}, J=12.0 \mathrm{~Hz}, \mathrm{NAP}), 4.89(1 \mathrm{H}, \mathrm{d}, J=12.0 \mathrm{~Hz}, \mathrm{NAP}), 7.29-7.48(9 \mathrm{H}$, m, TBPS, NAP), 7.70-7.86 (8H, m, TBPS, NAP); $\left.{ }^{13} \mathrm{C} \mathrm{NMR} \mathrm{(125} \mathrm{MHz,} \mathrm{CDCl}_{3}\right), \delta 10.9,12.5,12.7,13.6$, $16.0,18.18,18.20,19.4,22.6,24.3,26.9,28.1,28.2,31.6,32.9,35.0,38.0,38.6,42.4,46.1,46.7,56.2$, $67.3,67.7,68.4,72.1,72.5,73.7,74.3,77.8,77.9,80.7,82.6,84.3,84.4,96.2,107.8,125.6,125.9$, $126.0,126.2,127.3,127.4,127.7,127.8,127.9,129.3,132.8,133.2,133.8,134.2,135.7,136.07,136.09$, 136.5, 207.5; MALDI-TOF MS, calcd for $\mathrm{C}_{67} \mathrm{H}_{98} \mathrm{O}_{11} \mathrm{Si}_{2} \mathrm{Na} 1157.65\left(\mathrm{M}+\mathrm{Na}^{+}\right)$, found 1157.65. $42 \beta$ : colorless oil; $[\alpha]_{\mathrm{D}}{ }^{20}=-7.8\left(c 1.00, \mathrm{CHCl}_{3}\right)$; IR (film) $v 2930,2866,1727,1461,1101,1034,817 \mathrm{~cm}^{-1}$; ${ }^{1} \mathrm{H}$ NMR $\left(500 \mathrm{MHz}, \mathrm{CDCl}_{3}\right) \delta 0.93(3 \mathrm{H}, \mathrm{d}, J=6.5 \mathrm{~Hz}, \mathrm{Me} 57), 0.95(3 \mathrm{H}, \mathrm{d}, J=7.0 \mathrm{~Hz}, \mathrm{Me} 55), 1.02-$ $1.04(33 \mathrm{H}, \mathrm{m}, \mathrm{Me} 53, \mathrm{TBPS}, \mathrm{TIPS}), 1.06(3 \mathrm{H}, \mathrm{d}, J=6.5 \mathrm{~Hz}, \mathrm{Me} 56), 1.10$ (3H, d, $J=7.0 \mathrm{~Hz}, \mathrm{Me} 54)$, 1.51-1.60 (2H, m, H35, H48), 1.60-1.69 (2H, m, H37, H47), 1.69 (1H, ddd, J = 12.0, 12.0, $12.0 \mathrm{~Hz}$, H32), 1.76-1.83 (2H, m, H50, H51), 1.84 (1H, brd, $J=12.5$ Hz, H35), 1.87-1.97 (2H, m, H36, H50), $2.00(1 \mathrm{H}, \mathrm{brd}, J=14.0 \mathrm{~Hz}, \mathrm{H} 37), 2.03(1 \mathrm{H}, \mathrm{m}, \mathrm{H} 51), 2.08(1 \mathrm{H}, \mathrm{ddd}, J=12.0,5.0,5.0 \mathrm{~Hz}, \mathrm{H} 32), 2.41$ (1H, dd, $J=16.0,7.5 \mathrm{~Hz}, \mathrm{H} 40), 2.42-2.47$ (1H, m, H43), $2.84(1 \mathrm{H}, \mathrm{dd}, J=16.0,6.5 \mathrm{~Hz}, \mathrm{H} 40), 3.14$ (1H, ddd, $J=12.0,9.5,5.0 \mathrm{~Hz}, \mathrm{H} 33), 3.26(1 \mathrm{H}, \mathrm{dd}, J=10.0,10.0 \mathrm{~Hz}, \mathrm{H} 46), 3.29-3.36$ (2H, m, H34, H38), $3.36(3 \mathrm{H}, \mathrm{s}, \mathrm{MOM}), 3.55(1 \mathrm{H}, \mathrm{d}, J=11.0 \mathrm{~Hz}, \mathrm{H} 29), 3.58(1 \mathrm{H}, \mathrm{d}, J=8.5 \mathrm{~Hz}, \mathrm{H} 42), 3.68(1 \mathrm{H}, \mathrm{d}, J=11.0$ Hz, H29), 3.74 (1H, dd, $J=7.5,6.5$ Hz, H39), 3.84 (1H, ddd, $J=7.5,7.5,7.5$ Hz, H52), 3.92 (1H, ddd, $J=7.5,7.5,4.5 \mathrm{~Hz}, \mathrm{H} 52), 4.01(1 \mathrm{H}, \mathrm{s}, \mathrm{H} 44), 4.02(1 \mathrm{H}, \mathrm{d}, J=10.0 \mathrm{~Hz}, \mathrm{H} 45), 4.07(1 \mathrm{H}, \mathrm{dd}, J=12.0,5.0$ Hz, H31), $4.64(1 \mathrm{H}, \mathrm{d}, J=6.5 \mathrm{~Hz}, \mathrm{MOM}), 4.67(1 \mathrm{H}, \mathrm{d}, J=12.0 \mathrm{~Hz}, \mathrm{NAP}), 4.88(1 \mathrm{H}, \mathrm{d}, J=6.5 \mathrm{~Hz}$ 
MOM), $4.96(1 \mathrm{H}, \mathrm{d}, J=12.0 \mathrm{~Hz}, \mathrm{NAP}), 7.30-7.50$ (9H, m, TBPS, NAP), 7.69-7.84 (8H, m, TBPS, $\mathrm{NAP}) ;{ }^{13} \mathrm{C} \mathrm{NMR}\left(125 \mathrm{MHz}, \mathrm{CDCl}_{3}\right) \delta 12.2,12.5,12.8,13.6,15.9,18.20,18.21,19.4,24.3,26.9,27.8$, $28.7,29.1,29.2,29.35,29.43,29.7,35.0,36.5,37.7,39.9,42.1,45.6,47.4,56.2,67.27,67.32,68.4$, $72.9,73.5,74.0,77.8,80.8,81.0,82.2,82.5,83.5,84.6,98.8,107.9,125.6,125.9,126.0,126.1,127.3$, $127.4,127.6,127.78,127.84,129.3,132.8,133.3,133.8,134.3,135.7,136.1,136.8,208.7$; HRMS (MALDI), calcd for $\mathrm{C}_{67} \mathrm{H}_{98} \mathrm{O}_{11} \mathrm{Si}_{2} \mathrm{Na} 1157.6545\left(\mathrm{M}+\mathrm{Na}^{+}\right)$, found 1157.6521.

Isomerization of $\mathbf{4 2} \alpha$. To a solution of C42-epimer $\mathbf{4 2} \boldsymbol{\alpha}(22.7 \mathrm{mg}, 20.0 \mu \mathrm{mol})$ in $\mathrm{CH}_{2} \mathrm{Cl}_{2}(0.2 \mathrm{~mL})$ at room temperature was added DBU $(10 \mu \mathrm{l})$. After being stirred for $2 \mathrm{~h}$, the mixture was diluted with EtOAc and saturated aqueous $\mathrm{NH}_{4} \mathrm{Cl}$, and then extracted with hexane/EtOAc (x2). The organic layer was washed with saturated aqueous $\mathrm{NH}_{4} \mathrm{Cl}$ and brine, dried over $\mathrm{MgSO}_{4}$, and concentrated. The residue was purified through a pad of silica gel, and the resulting ketones were separated by HPLC (YMC-Pack SIL-06, 10x250 mm, UV $254 \mathrm{~nm}$, hexane/EtOAc 17:1, $5.0 \mathrm{~mL} / \mathrm{min})$ to give $\mathbf{4 2} \beta\left(\mathrm{t}_{\mathrm{R}}=21.3\right.$ min) and C42-epimer $\mathbf{4 2} \alpha\left(t_{R}=17.9 \mathrm{~min}\right)$. The undesired $\mathbf{4 2} \alpha$ was subjected to additional two cycles of the isomerization-separation sequence to give $\mathbf{4 2} \beta$ (13.6 $\mathrm{mg}, 12 \mu \mathrm{mol}, 60 \%$ for 3 cycles) and $\mathbf{4 2} \alpha(4.3$ $\mathrm{mg}, 4 \mu \mathrm{mol}, 19 \%$ for 3 cycles).

Alcohol 44. To a solution of enol ether $39(10.1 \mathrm{mg}, 9.0 \mu \mathrm{mol})$ in $\mathrm{CH}_{2} \mathrm{Cl}_{2}(0.40 \mathrm{~mL})$ at $-78{ }^{\circ} \mathrm{C}$ was added DMDO $(50 \mathrm{mM}$ in acetone, $0.36 \mathrm{~mL}, 18.0 \mu \mathrm{mol})$. The reaction mixture was allowed to warm to $-45^{\circ} \mathrm{C}$ over $30 \mathrm{~min}$, and concentrated at $0{ }^{\circ} \mathrm{C}$ to give epoxide 43 , which was used in the next reaction without purification: colorless oil; ${ }^{1} \mathrm{H}$ NMR $\left(500 \mathrm{MHz}, \mathrm{C}_{6} \mathrm{D}_{6}\right) \delta 0.88(3 \mathrm{H}, \mathrm{d}, J=7.0 \mathrm{~Hz}, \mathrm{Me} 57), 1.02$ 
(3H, d, $J=7.0 \mathrm{~Hz}, \mathrm{Me} 54), 1.08-1.14$ (30H, m, TBPS, TIPS), 1.16 (3H, d, $J=6.0 \mathrm{~Hz}, \mathrm{Me} 56), 1.24$ (3H, s, Me53), $1.42(1 \mathrm{H}, \mathrm{dq}, J=11.5,7.0 \mathrm{~Hz}, \mathrm{H} 48), 1.50(1 \mathrm{H}, \mathrm{m}, \mathrm{H} 51), 1.61(1 \mathrm{H}, \mathrm{m}, \mathrm{H} 35), 1.68(3 \mathrm{H}, \mathrm{d}, J=$ 7.0 Hz, Me55), 1.69-1.82 (3H, m, H37, H50x2), 1.83-1.93 (4H, m, H32, H36, H47, H51), 1.93-2.04 (3H, m, H35, H37, H40), 2.16 (1H, dq, $J=9.5,7.0, \mathrm{~Hz}, \mathrm{H} 43), 2.27$ (1H, ddd, $J=12.5,5.0,5.0 \mathrm{~Hz}$, H32), 2.49 (1H, ddd, $J=14.5,5.0,1.5 \mathrm{~Hz}, \mathrm{H} 40), 3.13$ (1H, ddd, $J=10.0,10.0,5.0 \mathrm{~Hz}, \mathrm{H} 33), 3.19$ (3H, s, MOM), $3.47(1 \mathrm{H}, \mathrm{ddd}, J=10.0,10.0,3.0 \mathrm{~Hz}, \mathrm{H} 34), 3.51(1 \mathrm{H}, \mathrm{dd}, J=9.5,9.5 \mathrm{~Hz}, \mathrm{H} 46), 3.56(1 \mathrm{H}, \mathrm{d}$, $J=1.5 \mathrm{~Hz}, \mathrm{H} 41), 3.65$ (1H, ddd, $J=9.5,9.5,2.5 \mathrm{~Hz}, \mathrm{H} 38), 3.72-3.80$ (2H, m, H39, H52), 3.78 (1H, d, $J$ $=11.0 \mathrm{~Hz}, \mathrm{H} 29), 3.86(1 \mathrm{H}, \mathrm{d}, J=11.0 \mathrm{~Hz}, \mathrm{H} 29), 3.91-3.98(2 \mathrm{H}, \mathrm{m}, \mathrm{H} 44, \mathrm{H} 52), 4.19$ (1H, dd, $J=12.0$, $5.0 \mathrm{~Hz}, \mathrm{H} 31), 4.36(1 \mathrm{H}, \mathrm{d}, J=9.5 \mathrm{~Hz}, \mathrm{H} 45), 4.52(1 \mathrm{H}, \mathrm{d}, J=6.5 \mathrm{~Hz}, \mathrm{MOM}), 4.76(1 \mathrm{H}, \mathrm{d}, J=12.0 \mathrm{~Hz}$ NAP), $5.05(1 \mathrm{H}, \mathrm{d}, J=6.5 \mathrm{~Hz}, \mathrm{MOM}), 5.10(1 \mathrm{H}, \mathrm{d}, J=12.0 \mathrm{~Hz}, \mathrm{NAP}), 7.22-7.34$ (8H, m, TBPS, NAP), 7.52-7.70 (4H, m, TBPS, NAP), 7.80-8.00 (5H, m, TBPS, NAP).

To a solution of epoxide 43 in THF $(0.80 \mathrm{~mL})$ at $0{ }^{\circ} \mathrm{C}$ was added $\mathrm{LiBHEt}_{3}(1 \mathrm{M}$ solution in THF, $360 \mu \mathrm{l}$, $360 \mu \mathrm{mol})$. After being stirred at $0{ }^{\circ} \mathrm{C}$ for $3 \mathrm{~h}$, the mixture was diluted with EtOAc. The organic layer was washed with saturated aqueous $\mathrm{NH}_{4} \mathrm{Cl}$ and brine, and dried over $\mathrm{MgSO}_{4}$. Concentration and flash column chromatography (hexane/EtOAc 20:1) gave alcohol 44 (8.7 mg, $7.7 \mu \mathrm{mol}$ ) in 85\% yield over 2 steps and its diastereomer $40(0.5 \mathrm{mg}, 0.4 \mu \mathrm{mol})$ in $4 \%$ yield over 2 steps. $\quad$ 44: colorless oil; $[\alpha]_{\mathrm{D}}^{25}=-24.9\left(c 1.00, \mathrm{CHCl}_{3}\right) ; \mathrm{IR}($ film $) \vee 2942,2867,1461,1384,1104 \mathrm{~cm}^{-1} ;{ }^{1} \mathrm{H} \mathrm{NMR}(500 \mathrm{MHz}$ $\left.\mathrm{CDCl}_{3}\right) \delta 0.92(3 \mathrm{H}, \mathrm{d}, J=6.5 \mathrm{~Hz}, \mathrm{Me} 57), 1.00-1.05$ (36H, m, Me53, Me56, TIPS, TBPS), 1.07 (3H, d, $J$ $=7.0 \mathrm{~Hz}, \mathrm{Me} 54), 1.18(3 \mathrm{H}, \mathrm{d}, J=7.0 \mathrm{~Hz}, \mathrm{Me} 55), 1.45-1.63(3 \mathrm{H}, \mathrm{m}, \mathrm{H} 35, \mathrm{H} 37, \mathrm{H} 48), 1.47(1 \mathrm{H}, \mathrm{dd}, J=$ 13.5, $13.5 \mathrm{~Hz}, \mathrm{H} 40), 1.68(1 \mathrm{H}, \mathrm{ddd}, J=12.0,12.0,12.0 \mathrm{~Hz}, \mathrm{H} 32), 1.72$ (1H, m, H47), 1.77-1.87 (5H, m, H35, H36, H37, H50, H51), 1.97 (1H, m, H50), 2.03 (1H, m, H51), 2.10 (1H, ddd, J = 12.0, 5.0, 5.0 Hz, 
H32), $2.13(1 \mathrm{H}, \mathrm{ddd}, J=13.5,4.5,3.0 \mathrm{~Hz}, \mathrm{H} 40), 2.19(1 \mathrm{H}, \mathrm{dq}, J=7.5,7.0 \mathrm{~Hz}, \mathrm{H} 43), 3.03(1 \mathrm{H}, \mathrm{dd}, J=$ 10.0, $10.0 \mathrm{~Hz}, \mathrm{H} 46), 3.12$ (1H, m, H38), 3.15 (1H, ddd, $J=12.0,12.0,5.0 \mathrm{~Hz}, \mathrm{H} 33), 3.28(1 \mathrm{H}, \mathrm{d}, J=$ $7.5 \mathrm{~Hz}, \mathrm{H} 42), 3.31$ (1H, ddd, $J=12.0,12.0,2.5 \mathrm{~Hz}, \mathrm{H} 34), 3.35$ (3H, s, MOM), 3.51 (1H, m, H39), 3.52 $(1 \mathrm{H}, \mathrm{d}, J=10.5 \mathrm{~Hz}, \mathrm{H} 29), 3.68(1 \mathrm{H}, \mathrm{d}, J=10.5 \mathrm{~Hz}, \mathrm{H} 29), 3.81(1 \mathrm{H}, \mathrm{d}, J=3.0 \mathrm{~Hz}, \mathrm{H} 41), 3.85$ (1H, ddd, $J=7.0,7.0,7.0 \mathrm{~Hz}, \mathrm{H} 52), 3.89$ (1H, ddd, $J=7.0,7.0,5.0 \mathrm{~Hz}, \mathrm{H} 52), 3.97-4.00$ (2H, m, H44, OH41), $4.07(1 \mathrm{H}, \mathrm{dd}, J=12.0,5.0 \mathrm{~Hz}, \mathrm{H} 31), 4.30(1 \mathrm{H}, \mathrm{dd}, J=10.0,1.5 \mathrm{~Hz}, \mathrm{H} 45), 4.55(1 \mathrm{H}, \mathrm{d}, J=11.5 \mathrm{~Hz}$, NAP), $4.65(1 \mathrm{H}, \mathrm{d}, J=7.0 \mathrm{~Hz}, \mathrm{MOM}), 4.81(1 \mathrm{H}, \mathrm{d}, J=7.0 \mathrm{~Hz}, \mathrm{MOM}), 4.88(1 \mathrm{H}, \mathrm{d}, J=11.5 \mathrm{~Hz}, \mathrm{NAP})$, 7.31-7.48 (9H, m, NAP, TBPS), 7.70-7.83 (8H, m, NAP, TBPS); ${ }^{13} \mathrm{C}$ NMR (125 MHz, $\left.\mathrm{CDCl}_{3}\right) \delta 12.5$, $12.6,12.8,13.5,16.0,18.2,18.3,19.4,24.4,26.9,27.9,28.6,35.1,36.1,38.4,39.8,39.9,41.8,46.4$, $46.6,56.3,65.9,67.4,67.9,68.5,69.9,71.8,73.8,74.9,77.7,81.0,81.6,81.9,82.4,83.9,98.5,107.7$, $125.88,125.94,126.1,126.7,127.3,127.4,127.7,127.9,128.2,129.29,129.31,133.0,133.3,133.8$, 134.5, 135.5, 135.7, 136.2; HRMS (MALDI), calcd for $\mathrm{C}_{67} \mathrm{H}_{100} \mathrm{O}_{11} \mathrm{Si}_{2} \mathrm{Na} 1159.6702\left(\mathrm{M}+\mathrm{Na}^{+}\right)$, found 1159.6721.

Ketone 42 $\beta$. To a solution of alcohol $44(37.5 \mathrm{mg}, 32.9 \mu \mathrm{mol})$ in $\mathrm{CH}_{2} \mathrm{Cl}_{2}(3.3 \mathrm{~mL})$ at $0{ }^{\circ} \mathrm{C}$ was added Dess-Martin periodinane $(56 \mathrm{mg}, 132 \mu \mathrm{mol})$. After being stirred at room temperature for $1 \mathrm{~h}$, the reaction mixture was quenched with saturated aqueous $\mathrm{Na}_{2} \mathrm{~S}_{2} \mathrm{O}_{3}$ and aqueous $\mathrm{NH}_{4} \mathrm{Cl}$, and then extracted with hexane/EtOAc (x2). The organic layer was washed with brine, and dried over $\mathrm{MgSO}_{4}$. Concentration and flash column chromatography (hexane/EtOAc 1:0-20:1) gave ketone 42 $\beta$ (35.5 mg, $31.3 \mu \mathrm{mol})$ in $95 \%$ yield. 
Methylacetal 46. To a solution of ketone $42 \beta(14.3 \mathrm{mg}, 12.6 \mu \mathrm{mol})$ and $\mathrm{CH}(\mathrm{OMe})_{3} \quad(15 \mu \mathrm{l})$ in hexane $(0.30 \mathrm{~mL})$ at room temperature was added $\mathrm{TfOH}\left(0.125 \mathrm{M}\right.$ in $\left.\mathrm{CH}_{2} \mathrm{Cl}_{2}, 6 \mu \mathrm{l}, 0.74 \mu \mathrm{mol}\right)$. After being stirred at room temperature for $30 \mathrm{~h}$, the reaction mixture was quenched with saturated aqueous $\mathrm{NaHCO}_{3}$, and extracted with hexane/EtOAc (x2). The organic layer was washed with brine, and dried over $\mathrm{MgSO}_{4}$. Concentration and flash column chromatography (hexane/EtOAc 1:0-50:1) gave methylacetal $46(8.6 \mathrm{mg}, 7.8 \mu \mathrm{mol})$ in $62 \%$ yield: colorless oil; $[\alpha]_{\mathrm{D}}{ }^{19}=11.4\left(c 0.27, \mathrm{CHCl}_{3}\right)$; IR (film) $v$ 3735, 2944, 2867, 1461, $1104 \mathrm{~cm}^{-1} ;{ }^{1} \mathrm{H}$ NMR (500 MHz, $\left.\mathrm{CDCl}_{3}\right) \delta 0.92(3 \mathrm{H}, \mathrm{d}, J=7.0 \mathrm{~Hz}, \mathrm{Me} 57)$, 1.00-1.06 (33H, m, Me53, TBPS, TIPS), $1.07(3 \mathrm{H}, \mathrm{d}, J=7.5 \mathrm{~Hz}, \mathrm{Me} 55), 1.08(3 \mathrm{H}, \mathrm{d}, J=7.0 \mathrm{~Hz}$, Me54), $1.14(3 \mathrm{H}, \mathrm{d}, J=6.5 \mathrm{~Hz}, \mathrm{Me} 56), 1.49$ (1H, m, H35), $1.51(1 \mathrm{H}, \mathrm{dd}, J=14.0,14.0,14.0 \mathrm{~Hz}, \mathrm{H} 40)$, 1.55-1.71 (3H, m, H37, H47, H48), 1.73 (1H, ddd, $J=12.0,12.0,12.0 \mathrm{~Hz}, \mathrm{H} 32), 1.76-1.89$ (5H, m, H35, H36, H37, H50, H51), 1.96-2.05 (2H, m, H50, H51), 2.09 (1H, ddd, J = 12.0, 4.5, 4.5 Hz, H32), $2.17(1 \mathrm{H}, \mathrm{dqd}, J=9.0,7.5,2.0 \mathrm{~Hz}, \mathrm{H} 43), 2.45(1 \mathrm{H}, \mathrm{dd}, J=14.0,4.5 \mathrm{~Hz}, \mathrm{H} 40), 2.92(1 \mathrm{H}, \mathrm{d}, J=9.0 \mathrm{~Hz}$, H42), 3.03 (1H, ddd, $J=9.0,9.0,3.0 \mathrm{~Hz}, \mathrm{H} 38), 3.09$ (1H, ddd, $J=12.0,9.5,4.5 \mathrm{~Hz}, \mathrm{H} 33), 3.23(1 \mathrm{H}$, ddd, $J=14.0,9.0,4.5 \mathrm{~Hz}, \mathrm{H} 39), 3.33(1 \mathrm{H}, \mathrm{m}, \mathrm{H} 34), 3.34(3 \mathrm{H}, \mathrm{s}, \mathrm{MeO}), 3.39(1 \mathrm{H}, \mathrm{dd}, J=2.0,2.0 \mathrm{~Hz}$, H44), $3.55(1 \mathrm{H}, \mathrm{d}, J=11.0 \mathrm{~Hz}, \mathrm{H} 29), 3.59(1 \mathrm{H}, \mathrm{dd}, J=9.0,2.0 \mathrm{~Hz}, \mathrm{H} 45), 3.66(1 \mathrm{H}, \mathrm{dd}, J=9.0,9.0 \mathrm{~Hz}$, H46), 3.68 (1H, d, $J=11.0$ Hz, H29), 3.83 (1H, m, H52), 3.90 (1H, m, H52), 4.06 (1H, dd, $J=12.0,4.5$ Hz, H31), $4.81(1 \mathrm{H}, \mathrm{d}, J=12.0 \mathrm{~Hz}, \mathrm{NAP}), 4.82(1 \mathrm{H}, \mathrm{d}, J=12.0 \mathrm{~Hz}, \mathrm{NAP}), 7.31-7.52$ (9H, m, TBPS, NAP), 7.70-7.83 (8H, m, TBPS, NAP); ${ }^{13} \mathrm{C}$ NMR (125 MHz, $\left.\mathrm{CDCl}_{3}\right) \delta 12.5,12.8,13.5,16.8,18.2,19.4$, 20.2, 24.6, 26.8, 27.4, 28.4, 29.7, 35.1, 37.1, 38.1, 38.6, 40.1, 40.8, 45.3, 46.5, 48.4, 67.5, 67.7, 68.5, $69.0,72.6,72.8,73.5,77.7,81.4,82.0,83.8,84.3,85.2,99.1,107.8,125.4,125.7,125.8,125.9,127.3$, $127.4,127.5,127.6,127.7,129.30,129.34,132.7,133.3,133.7,134.4,135.7,136.1,137.5$; HRMS 
(MALDI), calcd for $\mathrm{C}_{66} \mathrm{H}_{96} \mathrm{O}_{10} \mathrm{Si}_{2} \mathrm{Na} 1127.6440\left(\mathrm{M}+\mathrm{Na}^{+}\right)$, found 1127.6432.

HIJKLM ring fragment 47. To a solution of methylacetal 46 (15.5 mg, $14.0 \mu \mathrm{mol}), \mathrm{Et}_{3} \mathrm{SiH}(0.22 \mathrm{~mL}$, $1.4 \mathrm{mmol})$ and powdered MS4A $(10 \mathrm{mg})$ in $\mathrm{CH}_{2} \mathrm{Cl}_{2}(1.5 \mathrm{~mL})$ at $-62{ }^{\circ} \mathrm{C}$ was added $\mathrm{BF}_{3} \cdot \mathrm{OEt}_{2}(10 \%$ solution in $\left.\mathrm{CH}_{2} \mathrm{Cl}_{2}, 55 \mu \mathrm{l}, 45 \mu \mathrm{mol}\right)$. The reaction mixture was allowed to warm to $-20{ }^{\circ} \mathrm{C}$ over $1 \mathrm{~h}$, and then quenched with $1 \% \mathrm{Et}_{3} \mathrm{~N}$-hexane and aqueous $\mathrm{NaHCO}_{3}$. The mixture was extracted with hexane/EtOAc (x2), the organic layer was washed with brine, and dried over $\mathrm{MgSO}_{4}$. Concentration and flash column chromatography (hexane/EtOAc 1:0-20:1) gave HIJKLM ring fragment 47 (12.3 mg, $11.4 \mu \mathrm{mol})$ in $81 \%$ yield: colorless oil; $[\alpha]_{\mathrm{D}}{ }^{20}=-2.7\left(c 0.94, \mathrm{CHCl}_{3}\right)$; IR (film) $v 2941,1602,1462,1382$, 1258, 1102, $976 \mathrm{~cm}^{-1} ;{ }^{1} \mathrm{H}$ NMR $\left(500 \mathrm{MHz}, \mathrm{CDCl}_{3}\right) \delta 0.92(3 \mathrm{H}, \mathrm{d}, J=6.0 \mathrm{~Hz}, \mathrm{Me} 57), 1.02-1.09(39 \mathrm{H}$, m, Me53, Me54, Me56, TIPS, TBPS), 1.13 (3H, d, $J=7.0 \mathrm{~Hz}, \mathrm{Me} 55), 1.42(1 \mathrm{H}, \mathrm{ddd}, J=11.5,11.5$, $11.5 \mathrm{~Hz}, \mathrm{H} 40), 1.50-1.60$ (4H, m, H35, H37, H47, H48), 1.71 (1H, ddd, $J=11.5,11.5,11.5$ Hz, H32), 1.77-1.89 (5H, m, H35, H36, H37, H50, H51), 1.95-2.00 (2H, m, H50, H51), 2.10 (1H, ddd, $J=11.5$, 4.5, 4.5 Hz, H32), 2.21 (1H, m, H43), 2.25 (1H, ddd, $J=11.5,4.5,4.5 \mathrm{~Hz}, \mathrm{H} 40), 2.88$ (1H, dd, $J=9.5$, $4.5 \mathrm{~Hz}, \mathrm{H} 42), 3.01(1 \mathrm{H}, \mathrm{ddd}, J=9.5,9.5,3.0 \mathrm{~Hz}, \mathrm{H} 38), 3.10(1 \mathrm{H}, \mathrm{ddd}, J=11.5,10.0,4.5 \mathrm{~Hz}, \mathrm{H} 33)$, $3.15(1 \mathrm{H}, \mathrm{ddd}, J=11.5,9.5,4.5 \mathrm{~Hz}, \mathrm{H} 39), 3.34(1 \mathrm{H}, \mathrm{ddd}, J=10.0,10.0,3.0 \mathrm{~Hz}, \mathrm{H} 34), 3.44(1 \mathrm{H}, \mathrm{dd}, J=$ 9.5, 9.5 Hz, H46), 3.50 (1H, d, $J=3.0 \mathrm{~Hz}, \mathrm{H} 44), 3.54$ (1H, d, $J=11.0 \mathrm{~Hz}, \mathrm{H} 29), 3.68$ (1H, d, $J=11.0$ Hz, H29), $3.69(1 \mathrm{H}, \mathrm{d}, J=9.5 \mathrm{~Hz}, \mathrm{H} 45), 3.76(1 \mathrm{H}, \mathrm{ddd}, J=7.5,7.5,7.5 \mathrm{~Hz}, \mathrm{H} 52), 3.86-3.92(2 \mathrm{H}, \mathrm{m}$, H41, H52), 4.07 (1H, dd, $J=11.5,4.5 \mathrm{~Hz}, \mathrm{H} 31), 4.81(1 \mathrm{H}, \mathrm{d}, J=12.5 \mathrm{~Hz}, \mathrm{NAP}), 4.84(1 \mathrm{H}, \mathrm{d}, J=12.5$ Hz, NAP), 7.32-7.48 (8H, m, NAP, TBPS), 7.53-7.56 (1H, m, NAP), 7.72-7.77 (4H, m, TBPS), 7.81-7.84 (4H, m, NAP); ${ }^{13} \mathrm{C}$ NMR (125 MHz, $\left.\mathrm{CDCl}_{3}\right) \delta 12.3,12.5,12.8,16.0,17.7,18.23,18.24,19.4$, 
20.0, 24.4, 26.9, 27.5, 28.3, 28.9, 30.4, 35.1, 38.1, 38.6, 40.3, 40.7, 41.9, 46.5, 67.4, 68.5, 71.8, 72.2, $73.4,74.2,77.6,78.0,81.0,83.2,83.8,84.7,86.8,108.3,125.6,125.9,126.06,126.09,127.3,127.4$, $127.7,127.8,128.8,129.30,129.31,130.9,132.8,133.2,133.7,134.4,135.7,136.1,137.0 ;$ HRMS (FAB), calcd for $\mathrm{C}_{65} \mathrm{H}_{94} \mathrm{O}_{9} \mathrm{Si}_{2} \mathrm{Na} 1097.6329\left(\mathrm{M}+\mathrm{Na}^{+}\right)$, found 1097.6333.

Alcohol 48. To a solution of HIJKLM ring fragment $47(20 \mathrm{mg}, 18.6 \mu \mathrm{mol})$ in a plastic test tube at room temperature was added buffered pyridinium hydrofluoride [1.0 mL, a mixture of THF (1.0 mL), pyridine $(0.57 \mathrm{~mL})$ and pyridinium hydrofluoride $(0.2 \mathrm{~mL})]$. After being stirred at room temperature for $12 \mathrm{~h}$, the reaction mixture was quenched with aqueous $\mathrm{CuSO}_{4}$, and extracted with hexane/EtOAc (x2). The organic layer was washed with brine, and dried over $\mathrm{Na}_{2} \mathrm{SO}_{4}$. Concentration and flash column chromatography (hexane/EtOAc 1:0-10:1) gave alcohol 48 (14.1 mg, 16.9 umol) in 91\% yield: colorless oil; $[\alpha]_{\mathrm{D}}{ }^{21}=-0.2\left(c 0.91, \mathrm{CHCl}_{3}\right) ; \mathrm{IR}(\mathrm{film}) \vee 3502,2928,1602,1461,1271,1100,975 \mathrm{~cm}^{-1}$; ${ }^{1} \mathrm{H}$ NMR $\left(500 \mathrm{MHz}, \mathrm{CDCl}_{3}\right) \delta 0.91(3 \mathrm{H}, \mathrm{d}, J=6.5 \mathrm{~Hz}, \mathrm{Me} 57), 1.01-1.08$ (27H, m, Me54, Me56, TIPS), 1.09 (3H, s, Me53) $1.12(3 \mathrm{H}, \mathrm{d}, J=7.0 \mathrm{~Hz}, \mathrm{Me} 55), 1.35-1.48$ (2H, m, H35, H40), 1.53-1.59 (3H, m, H37, H47, H48), 1.69 (1H, ddd, $J=12.0,12.0,12.0$ Hz, H32), 1.74-1.88 (5H, m, H35, H36, H37, H50, H51), 1.94-2.01 (2H, m, H50, H51), 2.06 (1H, ddd, J = 12.0, 5.0, 5.0 Hz, H32), 2.19 (1H, m, H43), 2.23 (1H, ddd, $J=12.0,5.0,5.0 \mathrm{~Hz}, \mathrm{H} 40), 2.87(1 \mathrm{H}, \mathrm{dd}, J=9.5,5.0 \mathrm{~Hz}, \mathrm{H} 42), 2.99(1 \mathrm{H}, \mathrm{ddd}, J=9.5,9.5$, $3.0 \mathrm{~Hz}, \mathrm{H} 38), 3.06-3.13$ (2H, m, H33, H39), 3.30 (1H, ddd, $J=9.5,9.5,3.0 \mathrm{~Hz}, \mathrm{H} 34), 3.42(1 \mathrm{H}, \mathrm{d}, J=$ $11.5 \mathrm{~Hz}, \mathrm{H} 29), 3.43$ (1H, dd, $J=9.5,9.5 \mathrm{~Hz}, \mathrm{H} 46), 3.50(1 \mathrm{H}, \mathrm{d}, J=3.0 \mathrm{~Hz}, \mathrm{H} 44), 3.56(1 \mathrm{H}, \mathrm{d}, J=11.5$ Hz, H29), $3.68(1 \mathrm{H}, \mathrm{d}, J=9.5 \mathrm{~Hz}, \mathrm{H} 45), 3.76(1 \mathrm{H}, \mathrm{ddd}, J=7.5,7.5,7.5 \mathrm{~Hz}, \mathrm{H} 52), 3.85-3.90(2 \mathrm{H}, \mathrm{m}$, H41, H52), 3.91 (1H, dd, $J=12.0,5.0 \mathrm{~Hz}, \mathrm{H} 31), 4.80(1 \mathrm{H}, \mathrm{d}, J=12.5 \mathrm{~Hz}, \mathrm{NAP}), 4.83(1 \mathrm{H}, \mathrm{d}, J=12.5$ 
$\mathrm{Hz}, \mathrm{NAP}), 7.45-7.54$ (3H, m, NAP), 7.80-7.85 (4H, m, NAP); ${ }^{13} \mathrm{C}$ NMR $\left(125 \mathrm{MHz}, \mathrm{CDCl}_{3}\right) \delta 12.7$, $13.0,13.4,15.9,18.1,18.2,19.9,23.0,23.7,24.4,27.6,28.9,30.3,35.1,37.9,38.6,38.7,40.4,40.6$, $41.9,46.1,67.4,67.5,71.9,72.2,73.4,74.1,78.0,80.9,84.7,86.8,108.3,125.6,125.9,126.0,126.1$, 127.7, 127.8, 128.9, 130.9, 132.4, 132.8, 133.2, 136.9; HRMS (MALDI), calcd for $\mathrm{C}_{49} \mathrm{H}_{76} \mathrm{O}_{9} \mathrm{SiNa}$ $859.5156\left(\mathrm{M}+\mathrm{Na}^{+}\right)$, found 859.5151.

Homoallylic alcohol 50. To a solution of alcohol $48(13.0 \mathrm{mg}, 15.5 \mu \mathrm{mol})$ and $\mathrm{Et}_{3} \mathrm{~N}(216 \mu \mathrm{l}, 1.55$ mmol) in $\mathrm{CH}_{2} \mathrm{Cl}_{2}(0.60 \mathrm{~mL})$ and DMSO $(0.30 \mathrm{~mL})$ at $0{ }^{\circ} \mathrm{C}$ was added $\mathrm{SO}_{3} \cdot \mathrm{Py}(124 \mathrm{mg}, 0.78 \mathrm{mmol})$. After being stirred at $0{ }^{\circ} \mathrm{C}$ for $2 \mathrm{~h}$, the reaction mixture was quenched with aqueous $\mathrm{NH}_{4} \mathrm{Cl}$, and extracted with hexane/EtOAc (x2). The organic layer was washed with saturated aqueous $\mathrm{NaHCO}_{3}$ and brine, and dried over $\mathrm{MgSO}_{4}$. Concentration gave aldehyde 49, which was used in the next reaction without further purification.

$\mathrm{MgBr}_{2}$ was prepared by stirring $\mathrm{Mg}$ turnings $(10 \mathrm{mg}, 388 \mu \mathrm{mol})$ and dibromoethane $(33 \mu \mathrm{l}, 388 \mu \mathrm{mol})$ in $\mathrm{Et}_{2} \mathrm{O}(0.40 \mathrm{~mL})$ for $30 \mathrm{~min}$ at $0{ }^{\circ} \mathrm{C}$ and following evaporation. To the solid of $\mathrm{MgBr}_{2}$ was added aldehyde 49 in $\mathrm{CH}_{2} \mathrm{Cl}_{2}(1.0 \mathrm{~mL})$ and the resulting mixture was cooled to $-100{ }^{\circ} \mathrm{C}$. The solution was treated with allyltributyltin $(24 \mu \mathrm{l}, 78 \mu \mathrm{mol})$, and allowed to warm to $0{ }^{\circ} \mathrm{C}$ over $16 \mathrm{~h}$. The reaction mixture was then quenched with saturated aqueous $\mathrm{NH}_{4} \mathrm{Cl}$, and extracted with hexane/EtOAc (x2). The organic layer was washed with brine, dried over $\mathrm{Na}_{2} \mathrm{SO}_{4}$, and concentrated to give homoallylic alcohol 50 along with their diastereomers at C49 and C29, which was used in the next reaction directly. To a solution of the homoallylic alcohols in $\left(\mathrm{CH}_{2} \mathrm{Cl}\right)_{2}(1.0 \mathrm{~mL})$ at room temperature was added CSA $(4.0$ $\mathrm{mg}, 15.5 \mu \mathrm{mol})$. After being stirred at room temperature for $3 \mathrm{~h}$, the reaction mixture was quenched 
with aqueous $\mathrm{NaHCO}_{3}$, and extracted with hexane/EtOAc (x2). The organic layer was washed with brine, and dried over $\mathrm{Na}_{2} \mathrm{SO}_{4}$. Concentration and flash column chromatography (hexane/EtOAc 1:0-30:1) gave homoallylic alcohol $50(9.9 \mathrm{mg}, 11.3 \mu \mathrm{mol})$ in $73 \%$ yield over 3 steps and C29-epimer $(1.7 \mathrm{mg}, 1.9 \mu \mathrm{mol})$ in $12 \%$ yield over 3 steps. 50: colorless oil; $[\alpha]_{\mathrm{D}}{ }^{21}=7.5\left(c 1.00, \mathrm{CHCl}_{3}\right)$; IR (film) $v$ 2927, 1640, 1460, 1379, 1098, 918, $883 \mathrm{~cm}^{-1} ;{ }^{1} \mathrm{H}$ NMR $\left(500 \mathrm{MHz}, \mathrm{CDCl}_{3}\right) \delta 0.91(3 \mathrm{H}, \mathrm{d}, J=6.0 \mathrm{~Hz}$, Me57), 1.06 (27H, m, Me54, Me56, TIPS), 1.09 (3H, s, Me53) 1.19 (3H, d, $J=8.0 \mathrm{~Hz}, \mathrm{Me} 55), 1.40$ (1H, ddd, $J=12.0,12.0,12.0 \mathrm{~Hz}, \mathrm{H} 40), 1.46-1.58(4 \mathrm{H}, \mathrm{m}, \mathrm{H} 35, \mathrm{H} 37, \mathrm{H} 47, \mathrm{H} 48), 1.69(1 \mathrm{H}, \mathrm{ddd}, J=12.0$, 12.0, 12.0 Hz, H32), 1.73-1.80 (2H, m, H35, H51), 1.80-1.87 (3H, m, H36, H37, H50), 1.94-2.01 (3H, m, H50, H51, OH29), 2.09 (1H, ddd, $J=12.0,5.0,5.0$ Hz, H32), 2.17-2.24 (3H, m, H28, H40, H43), 2.36 (1H, m, H28), 2.86 (1H, dd, $J=10.0,4.0 \mathrm{~Hz}, \mathrm{H} 42), 2.98$ (1H, ddd, $J=10.0,10.0,3.0 \mathrm{~Hz}, \mathrm{H} 38)$, 3.05 (1H, m, H33), 3.10 (1H, m, H39), 3.31 (1H, ddd, $J=10.0,10.0,3.5 \mathrm{~Hz}, \mathrm{H} 34), 3.43$ (1H, dd, $J=9.5$, $9.5 \mathrm{~Hz}, \mathrm{H} 46), 3.49$ (1H, d, $J=2.5 \mathrm{~Hz}, \mathrm{H} 44), 3.61(1 \mathrm{H}, \mathrm{ddd}, J=10.0,10.0,3.0 \mathrm{~Hz}, \mathrm{H} 29), 3.67(1 \mathrm{H}, \mathrm{d}, J$ $=9.5 \mathrm{~Hz}, \mathrm{H} 45), 3.76(1 \mathrm{H}, \mathrm{ddd}, J=7.5,7.5,7.5 \mathrm{~Hz}, \mathrm{H} 52), 3.84-3.90$ (2H, m, H41, H52), 4.16 (1H, dd, $J$ $=12.0,5.0 \mathrm{~Hz}, \mathrm{H} 31), 4.79(1 \mathrm{H}, \mathrm{d}, J=12.0 \mathrm{~Hz}, \mathrm{NAP}), 4.83(1 \mathrm{H}, \mathrm{d}, J=12.0 \mathrm{~Hz}, \mathrm{NAP}), 5.06(1 \mathrm{H}, \mathrm{dd}, J=$ 10.0, 2.0 Hz, H26), 5.09 (1H, dd, $J=17.0,2.0 \mathrm{~Hz}, \mathrm{H} 26), 5.93$ (1H, ddt, $J=17.0,10.0,7.0 \mathrm{~Hz}, \mathrm{H} 27)$, 7.44-7.54 (3H, m, NAP), 7.80-7.85 (4H, m, NAP); ${ }^{13} \mathrm{C}$ NMR (125 MHz, $\left.\mathrm{CDCl}_{3}\right) \delta 12.8,13.5,13.6$, $16.0,18.1,18.2,19.9,24.4,27.5,29.7,35.1,35.8,38.0,38.6,40.3,40.6,41.9,67.4,67.7,71.9,72.2$, 72.7, 73.3, 74.2, 78.0, 78.2, 80.9, 83.0, 83.2, 84.7, 86.8, 108.3, 116.5, 125.6, 125.9, 126.0, 126.1, 127.7, 127.8, 132.8, 133.2, 136.5, 136.9; MALDI-TOF MS, calcd for $\mathrm{C}_{52} \mathrm{H}_{80} \mathrm{O}_{9} \mathrm{SiNa} 899.54\left(\mathrm{M}+\mathrm{Na}^{+}\right)$, found 899.54 . 
Right wing 13 of CTX3C. To a solution of homoallylic alcohol 50 (25 mg, $28.5 \mu \mathrm{mol}), \mathrm{NaH}$ (14 mg, $0.57 \mathrm{mmol})$ and TBAI $(11 \mathrm{mg}, 28.5 \mu \mathrm{mol})$ in THF/DMF $(3: 1,2 \mathrm{~mL})$ at room temperature was added NAPBr $(29 \mathrm{mg}, 129 \mu \mathrm{mol})$. After being stirred at room temperature for $4 \mathrm{~h}$, the reaction mixture was quenched with $\mathrm{MeOH}$ and aqueous $\mathrm{NH}_{4} \mathrm{Cl}$, and extracted with hexane/EtOAc (x2). The organic layer was washed with brine, and dried over $\mathrm{Na}_{2} \mathrm{SO}_{4}$. Concentration and flash column chromatography (hexane/EtOAc 1:0-20:1) gave right wing 13 (28.3 mg, $27.8 \mu \mathrm{mol})$ in $98 \%$ yield: colorless oil; $[\alpha]_{\mathrm{D}}{ }^{26}=$ 1.9 (c $\left.0.95, \mathrm{CHCl}_{3}\right)$; IR (film) v 3056, 2944, 1509, 1460, 1098, 1027, $883 \mathrm{~cm}^{-1} ;{ }^{1} \mathrm{H}$ NMR $(500 \mathrm{MHz}$, $\left.\mathrm{CDCl}_{3}\right) \delta 0.91(3 \mathrm{H}, \mathrm{d}, J=6.0 \mathrm{~Hz}, \mathrm{Me} 57), 1.02-1.08$ (27H, m, Me54, Me56, TIPS), 1.12 (3H, d, $J=7.0$ Hz, Me55), 1.15 (3H, s, Me53), 1.40 (1H, ddd, $J=11.5,11.5,11.5$ Hz, H40), 1.51-1.62 (4H, m, H35, H37, H47, H48), 1.73 (1H, ddd, $J=12.0,12.0,12.0$ Hz, H32), 1.76-1.89 (5H, m, H35, H36, H37, H50, H51), 1.94-2.00 (2H, m, H50, H51), 2.18 (1H, ddd, $J=12.0,4.5,4.5 \mathrm{~Hz}, \mathrm{H} 32), 2.18$ (1H, m, H40), 2.21 (1H, m, H43), $2.53(1 \mathrm{H}, \mathrm{m}, \mathrm{H} 28), 2.54(1 \mathrm{H}, \mathrm{m}, \mathrm{H} 28), 2.86(1 \mathrm{H}, \mathrm{dd}, J=9.5,4.5 \mathrm{~Hz}, \mathrm{H} 42), 3.00$ (1H, ddd, $J=9.0,9.0,3.0 \mathrm{~Hz}, \mathrm{H} 38), 3.07(1 \mathrm{H}, \mathrm{m}, \mathrm{H} 33), 3.10(1 \mathrm{H}, \mathrm{m}, \mathrm{H} 39), 3.31(1 \mathrm{H}, \mathrm{ddd}, J=10.0,10.0,3.0 \mathrm{~Hz}$, H34), $3.43(1 \mathrm{H}, \mathrm{dd}, J=10.0,10.0 \mathrm{~Hz}, \mathrm{H} 46), 3.50(1 \mathrm{H}, \mathrm{d}, J=3.0 \mathrm{~Hz}, \mathrm{H} 44), 3.67(1 \mathrm{H}, \mathrm{dd}, J=8.0,4.0 \mathrm{~Hz}$, H29), $3.68(1 \mathrm{H}, \mathrm{d}, J=10.0 \mathrm{~Hz}, \mathrm{H} 45), 3.76(1 \mathrm{H}, \mathrm{ddd}, J=7.5,7.5,7.5 \mathrm{~Hz}, \mathrm{H} 52), 3.87$ (1H, m, H52), 3.89 $(1 \mathrm{H}, \mathrm{m}, \mathrm{H} 41), 4.27(1 \mathrm{H}, \mathrm{dd}, J=12.0,4.5 \mathrm{~Hz}, \mathrm{H} 31), 4.74(1 \mathrm{H}, \mathrm{d}, J=12.0 \mathrm{~Hz}, \mathrm{NAP}), 4.80(1 \mathrm{H}, \mathrm{d}, J=$ $12.0 \mathrm{~Hz}, \mathrm{NAP}), 4.83(1 \mathrm{H}, \mathrm{d}, J=12.0 \mathrm{~Hz}, \mathrm{NAP}), 4.84(1 \mathrm{H}, \mathrm{d}, J=12.0 \mathrm{~Hz}, \mathrm{NAP}), 5.02(1 \mathrm{H}, \mathrm{dd}, J=10.0$, $2.0 \mathrm{~Hz}, \mathrm{H} 26), 5.13(1 \mathrm{H}, \mathrm{dd}, J=17.0,2.0 \mathrm{~Hz}, \mathrm{H} 26), 6.00(1 \mathrm{H}, \mathrm{ddt}, J=17.0,10.0,7.0 \mathrm{~Hz}, \mathrm{H} 27)$, 7.40-7.55 (6H, m, NAP), 7.76-7.86 (8H, m, NAP); $\left.{ }^{13} \mathrm{C} \mathrm{NMR} \mathrm{(125} \mathrm{MHz,} \mathrm{CDCl}_{3}\right) \delta 13.1,13.5,13.9$, $16.0,18.31,18.32,19.9,24.3,27.6,28.1,33.3,35.0,38.4,38.6,40.3,40.6,41.9,45.5,46.0,67.4,68.4$, $71.5,71.8,72.2,73.5,74.2,78.0,80.0,80.9,82.0,83.0,83.1,84.7,86.7,108.3,115.9,125.4,125.6$, 
$125.73,125.75,125.9,126.02,126.08,127.56,127.65,127.69,127.74,127.8,132.7,132.8,133.2$,

133.3, 136.9, 137.0, 137.3; HRMS (FAB), calcd for $\mathrm{C}_{63} \mathrm{H}_{88} \mathrm{O}_{9} \mathrm{SiNa} 1039.6090\left(\mathrm{M}+\mathrm{Na}^{+}\right)$, found 1039.6071.

Ester 51. To a mixture of alcohol 9 (197 mg, $0.22 \mathrm{mmol})$, carboxylic acid 11 (144 mg, $0.23 \mathrm{mmol})$ and 2,4,6-trichlorobenzoyl chloride $(0.17 \mathrm{~mL}, 1.1 \mathrm{mmol})$ in toluene $(4.4 \mathrm{~mL})$ at $30{ }^{\circ} \mathrm{C}$ was added to DMAP (268 mg, $2.2 \mathrm{mmol}$ ). After being stirred at $30{ }^{\circ} \mathrm{C}$ for $18 \mathrm{~h}$, the reaction mixture was quenched with aqueous $\mathrm{NH}_{4} \mathrm{Cl}$, and extracted with EtOAc (x3). The organic layer was washed with brine, and dried over $\mathrm{MgSO}_{4}$. Concentration and flash column chromatography (hexane/EtOAc 25:1-10:1) gave ester $51(255 \mathrm{mg}, 0.17 \mathrm{mmol})$ in $77 \%$ yield: colorless oil; $[\alpha]_{\mathrm{D}}{ }^{17}=-63.3\left(c 1.33, \mathrm{CHCl}_{3}\right)$; IR (film) v 3054, 2940, 1730, 1462, 1098, $816 \mathrm{~cm}^{-1} ;{ }^{1} \mathrm{H}$ NMR (500 MHz, $\left.\mathrm{CDCl}_{3}\right) \delta 0.98(21 \mathrm{H}, \mathrm{s}, \mathrm{TIPS}), 1.03-1.08$ (21H, m, Me53, Me54, Me56, Me57, TBPS), 1.23 (3H, d, J = 7.0 Hz, Me55), 1.56-1.81 (5H, m, H32, H35, H37, H47, H48), 1.86-1.95 (4H, m, H35, H36, H37, H40), 2.03 (1H, dd, J = 12.0, 12.0 Hz, H40), $2.09(1 \mathrm{H}, \mathrm{dd}, J=13.5,6.5 \mathrm{~Hz}, \mathrm{H} 50), 2.16(1 \mathrm{H}, \mathrm{dd}, J=13.5,3.0 \mathrm{~Hz}, \mathrm{H} 50), 2.30(1 \mathrm{H}, \mathrm{ddd}, J=11.5,5.0$, $5.0 \mathrm{~Hz}, \mathrm{H} 32), 2.77(1 \mathrm{H}, \mathrm{ddd}, J=7.0,7.0,7.0 \mathrm{~Hz}, \mathrm{H} 43), 3.06(1 \mathrm{H}, \mathrm{dd}, J=10.0,10.0 \mathrm{~Hz}, \mathrm{H} 46)$, 3.28-3.40 (2H, m, H33, H34), 3.37 (3H, s, MOM), 3.57 (1H, d, $J=10.5 \mathrm{~Hz}, \mathrm{H} 29), 3.66(1 \mathrm{H}, \mathrm{d}, J=10.5$ Hz, H29), 3.90-3.92 (3H, m, H44, H45, H52), 3.99 (1H, d, $J=10.0$ Hz, H52), 4.01 (1H, dd, $J=11.5$, $5.0 \mathrm{~Hz}, \mathrm{H} 31), 4.11(1 \mathrm{H}, \mathrm{ddd}, J=9.5,9.5,1.5 \mathrm{~Hz}, \mathrm{H} 38), 4.29$ (1H, m, H51), 4.56-4.63 (5H, m, H39, H41, MOM, NAPx2), $4.68(1 \mathrm{H}, \mathrm{d}, J=11.5 \mathrm{~Hz}, \mathrm{NAP}), 4.77(1 \mathrm{H}, \mathrm{d}, J=6.5 \mathrm{~Hz}, \mathrm{MOM}), 4.86(1 \mathrm{H}, \mathrm{d}, J=$ $11.5 \mathrm{~Hz}, \mathrm{NAP}), 7.23-7.83(34 \mathrm{H}, \mathrm{m}, \mathrm{PhSx} 2, \mathrm{NAPx} 2, \mathrm{TBPS}) ;{ }^{13} \mathrm{C} \mathrm{NMR}\left(125 \mathrm{MHz}, \mathrm{CDCl}_{3}\right) \delta 12.4,12.6$, $13.6,14.4,15.8,18.2,19.3,26.1,26.8,28.3,29.6,37.2,39.8,40.2,41.3,41.9,42.6,44.2,53.7,56.4$, 
67.5, 68.6, 71.0, 71.4, 72.7, 72.8, 74.0, 76.4, 77.7, 78.6, 79.4, 80.4, 80.7, 82.3, 98.5, 108.5, 125.6, 125.7, $125.8,125.9,126.0,126.1,126.2,126.3,127.2,127.3,127.6,127.8,127.9,128.0,128.1,128.8,128.9$, $129.3,131.6,132.1,132.8,132.9,133.23,133.24,133.7,134.2,134.9,135.6,136.0,136.2,174.6$; HRMS (FAB), calcd for $\mathrm{C}_{90} \mathrm{H}_{116} \mathrm{O}_{12} \mathrm{~S}_{2} \mathrm{Si}_{2} \mathrm{Na}\left(\mathrm{M}+\mathrm{Na}^{+}\right) 1531.7344$, found 1531.7371.

Cyclic enol ether 52. Powdered MS4A (250 mg), Mg turnings (29 mg, $1.2 \mathrm{mmol})$, and $\mathrm{Cp}_{2} \mathrm{TiCl}_{2}(255$ $\mathrm{mg}, 1.0 \mathrm{mmol})$ were placed in a flask and dried with a heat gun under reduced pressure. THF $(3 \mathrm{~mL})$ and $\mathrm{P}(\mathrm{OEt})_{3}(150 \mu \mathrm{l}, 864 \mu \mathrm{mol})$ were added to this flask successively at room temperature, and the reaction mixture was stirred for $3 \mathrm{~h}$. To this mixture was added ester $\mathbf{5 2}(310 \mathrm{mg}, 0.2 \mathrm{mmol})$ in THF $(1.0 \mathrm{~mL})$ dropwise at room temperature. After being heated to reflux for $1 \mathrm{~h}$, the reaction was quenched with $1 \mathrm{M} \mathrm{NaOH}$ at room temperature and resulting insoluble solid was filtered off using Celite. The mixture was extracted with with $\mathrm{Et}_{2} \mathrm{O}(\mathrm{x} 2)$, and the organic layer was washed with brine. The violet organic layer was dried over $\mathrm{MgSO}_{4}$ overnight to give colorless solution. Concentration and flash column chromatography (hexane/EtOAc 1:0-20:1) gave cyclic enol ether 52 (174 mg, $0.13 \mathrm{mmol}$ ) in 68\% yield: colorless oil; $[\alpha]_{\mathrm{D}}{ }^{19}=-18.7\left(c 1.28, \mathrm{CHCl}_{3}\right)$; IR (film) $v 2931,1460,1104,1030,816,737$ $\mathrm{cm}^{-1}$; ${ }^{1} \mathrm{H}$ NMR (500 MHz, $\mathrm{CDCl}_{3}$ ) $\delta$ 1.02-1.04 (42H, m, TIPS, TBPS, Me53, Me54, Me56, Me57), 1.14 $(3 \mathrm{H}, \mathrm{d}, J=7.0 \mathrm{~Hz}, \mathrm{Me} 55), 1.45-1.55$ (2H, m, H37, H48), 1.62-1.74 (3H, m, H32, H35, H47), 1.85-1.94 (3H, m, H35, H36, H37), 2.04-2.17 (4H, m, H32, H40, H50x2), 2.21 (1H, ddd, $J=11.5,5.0,5.0 \mathrm{~Hz}$, H40), $2.48(1 \mathrm{H}, \mathrm{dq}, J=9.5,7.0 \mathrm{~Hz}, \mathrm{H} 43), 3.16-3.22(2 \mathrm{H}, \mathrm{m}, \mathrm{H} 33, \mathrm{H} 46), 3.35-3.40$ (2H, m, H38, H39), $3.39(3 \mathrm{H}, \mathrm{s}, \mathrm{MOM}), 3.48(1 \mathrm{H}, \mathrm{ddd}, J=10.0,10.0,2.0 \mathrm{~Hz}, \mathrm{H} 34), 3.54(1 \mathrm{H}, \mathrm{d}, J=9.5 \mathrm{~Hz}, \mathrm{H} 44), 3.57$ $(1 \mathrm{H}, \mathrm{d}, J=10.0 \mathrm{~Hz}, \mathrm{H} 29), 3.68(1 \mathrm{H}, \mathrm{d}, J=10.0 \mathrm{~Hz}, \mathrm{H} 29), 3.85(1 \mathrm{H}, \mathrm{dd}, J=9.5,5.0 \mathrm{~Hz}, \mathrm{H} 52), 3.91(1 \mathrm{H}$, 
$\mathrm{d}, J=9.5 \mathrm{~Hz}, \mathrm{H} 45), 4.01(1 \mathrm{H}, \mathrm{dd}, J=9.5,1.0 \mathrm{~Hz}, \mathrm{H} 52), 4.06(1 \mathrm{H}, \mathrm{dd}, J=11.5,5.0 \mathrm{~Hz}, \mathrm{H} 31), 4.32(1 \mathrm{H}$, m, H51), 4.45 (1H, dd, $J=5.0,1.5 \mathrm{~Hz}, \mathrm{H} 41), 4.61(1 \mathrm{H}, \mathrm{d}, J=6.0 \mathrm{~Hz}, \mathrm{MOM}), 4.62(2 \mathrm{H}, \mathrm{s}, \mathrm{NAP}), 4.63$ $(1 \mathrm{H}, \mathrm{d}, J=12.0 \mathrm{~Hz}, \mathrm{NAP}), 4.86(1 \mathrm{H}, \mathrm{d}, J=6.0 \mathrm{~Hz}, \mathrm{MOM}), 4.89(1 \mathrm{H}, \mathrm{d}, J=12.0 \mathrm{~Hz}, \mathrm{NAP}), 7.32-7.83$ (24H, m, TBPS, NAPx2); ${ }^{13} \mathrm{C}$ NMR $\left(125 \mathrm{MHz}, \mathrm{CDCl}_{3}\right) \delta 12.4,12.7,13.7,15.9,16.2,18.20,18.22,19.3$, $26.8,27.3,30.8,37.8,39.7,40.7,41.9,42.6,56.4,67.2,68.5,71.0,71.1,72.6,73.4,73.6,77.6,78.6$ $79.1,79.8,82.2,82.7,94.6,98.4,108.5,125.6,125.7,125.8,125.9,126.0,126.1,126.2,126.3,127.2$ $127.3,127.62,126.68,127.76,127.82,127.83,128.1,129.3,132.8,132.9,133.2,133.3,133.7,134.2$, 135.6, 135.7, 136.0, 136.6, 155.6; MALDI-TOF MS, calcd for $\mathrm{C}_{78} \mathrm{H}_{106} \mathrm{O}_{11} \mathrm{Si}_{2} \mathrm{Na}\left(\mathrm{M}+\mathrm{Na}^{+}\right)$1297.71, found 1297.73.

Alcohol 54. To a solution of enol ether $52(139.4 \mathrm{mg}, 109 \mu \mathrm{mol})$ in $\mathrm{CH}_{2} \mathrm{Cl}_{2}(3.6 \mathrm{~mL})$ at $-78{ }^{\circ} \mathrm{C}$ was added DMDO in acetone $(4.4 \mathrm{~mL}, 218 \mu \mathrm{mol})$. The reaction mixture was allowed to warm to $-45^{\circ} \mathrm{C}$ over $30 \mathrm{~min}$, and concentrated at $0{ }^{\circ} \mathrm{C}$ to give epoxide $\mathbf{5 3}$, which was directly used in the next reaction. To a solution of epoxide 53 in THF $(7.3 \mathrm{~mL})$ at $0{ }^{\circ} \mathrm{C}$ was added $\operatorname{LiBHEt}_{3}(1 \mathrm{M}$ solution in THF, $4.4 \mathrm{~mL}$, $4.4 \mathrm{mmol}$ ). After being stirred at $0{ }^{\circ} \mathrm{C}$ for $3 \mathrm{~h}$, the mixture was diluted with EtOAc. The organic layer was washed with saturated aqueous $\mathrm{NH}_{4} \mathrm{Cl}$ and brine, dried over $\mathrm{MgSO}_{4}$. Concentration and flash column chromatography (hexane/EtOAc 10:1) gave alcohol $\mathbf{5 4}$ (120 mg, $85 \mu \mathrm{mol}$ ) in $78 \%$ yield: colorless oil; $[\alpha]_{\mathrm{D}}{ }^{26}=-20.5\left(c 1.03, \mathrm{CHCl}_{3}\right) ; \mathrm{IR}($ film $) \vee 3408,2928,2865,1461,1104,1029,818,736$ $\mathrm{cm}^{-1} ;{ }^{1} \mathrm{H}$ NMR (500 MHz, $\mathrm{CDCl}_{3}$ ) $\delta$ 1.01-1.07 (42H, m, TIPS, TBPS, Me53, Me54, Me56, Me57), 1.15 (3H, d, $J=6.5 \mathrm{~Hz}, \mathrm{Me} 55), 1.41-1.54$ (4H, m, H35, H37, H40, H48), 1.67 (1H, ddd, $J=12.0,12.0,12.0$ Hz, H32), 1.73-1.86 (4H, m, H35, H36, H37, H47), 2.07-2.19 (5H, m, H32, H40, H43, H50x2), 3.03 
$(1 \mathrm{H}, \mathrm{dd}, J=10.0,10.0 \mathrm{~Hz}, \mathrm{H} 46), 3.09-3.16(2 \mathrm{H}, \mathrm{m}, \mathrm{H} 33, \mathrm{H} 38), 3.24(1 \mathrm{H}, \mathrm{d}, J=8.0 \mathrm{~Hz}, \mathrm{H} 42), 3.31(1 \mathrm{H}$, ddd, $J=10.0,10.0,3.0 \mathrm{~Hz}, \mathrm{H} 34), 3.35$ (3H, s, MOM), 3.50 (1H, ddd, $J=12.0,10.0,5.0 \mathrm{~Hz}, \mathrm{H} 39), 3.52$ $(1 \mathrm{H}, \mathrm{d}, J=10.0 \mathrm{~Hz}, \mathrm{H} 29), 3.67(1 \mathrm{H}, \mathrm{d}, J=10.0 \mathrm{~Hz}, \mathrm{H} 29), 3.74(1 \mathrm{H}, \mathrm{d}, J=2.5 \mathrm{~Hz}, \mathrm{H} 41), 3.89$ (1H, dd, $J$ $=10.0,5.0 \mathrm{~Hz}, \mathrm{H} 52), 3.92(1 \mathrm{H}, \mathrm{d}, J=2.5 \mathrm{~Hz}, \mathrm{OH} 41), 3.97(1 \mathrm{H}, \mathrm{s}, \mathrm{H} 44), 3.98(1 \mathrm{H}, \mathrm{d}, J=10.0 \mathrm{~Hz}, \mathrm{H} 52)$, $4.06(1 \mathrm{H}, \mathrm{dd}, J=12.0,5.0 \mathrm{~Hz}, \mathrm{H} 31), 4.28(1 \mathrm{H}, \mathrm{d}, J=10.0 \mathrm{~Hz}, \mathrm{H} 45), 4.32(1 \mathrm{H}, \mathrm{m}, \mathrm{H} 51), 4.54$ (1H, d, $J$ $=12.0 \mathrm{~Hz}, \mathrm{NAP}), 4.57(1 \mathrm{H}, \mathrm{d}, J=12.0 \mathrm{~Hz}, \mathrm{NAP}), 4.61(1 \mathrm{H}, \mathrm{d}, J=12.0 \mathrm{~Hz}, \mathrm{NAP}), 4.65(1 \mathrm{H}, \mathrm{d}, J=6.0$ Hz, MOM), $4.81(1 \mathrm{H}, \mathrm{d}, J=6.0 \mathrm{~Hz}, \mathrm{MOM}), 4.85(1 \mathrm{H}, \mathrm{d}, J=12.0 \mathrm{~Hz}, \mathrm{NAP}), 7.31-7.82(24 \mathrm{H}, \mathrm{m}$, NAPx2, TBPS); ${ }^{13} \mathrm{C}$ NMR (125 MHz, $\left.\mathrm{CDCl}_{3}\right) \delta 12.4,12.7,13.7,16.1,18.3,18.4,19.5,27.0,27.9,28.7$, $35.8,38.5,39.9,41.7,42.7,46.5,46.7,56.4,65.8,67.4,68.5,69.9,71.3,71.93,71.94,73.8,74.4,77.8$, $78.8,81.0,81.5,81.8,82.4,84.0,98.6,108.8,125.7,126.01,126.07,126.10,126.2,126.3,126.4,126.8$, $127.4,127.5,127.8,127.9,128.0,128.3,128.4,129.45,129.46,133.1,113.2,133.35,133.42,133.8$, $134.5,135.4,135.7,135.8,135.9,136.25,136.29 ;$ MALDI-TOF MS, calcd for $\mathrm{C}_{78} \mathrm{H}_{108} \mathrm{O}_{12} \mathrm{Si}_{2} \mathrm{Na}$ $\left(\mathrm{M}+\mathrm{Na}^{+}\right)$1315.72, found 1315.71

Ketone 55. To a solution of alcohol $54(37.5 \mathrm{mg}, 32.9 \mu \mathrm{mol})$ in $\mathrm{CH}_{2} \mathrm{Cl}_{2}(3.3 \mathrm{~mL})$ at $0{ }^{\circ} \mathrm{C}$ was added Dess-Martin periodinane $(56 \mathrm{mg}, 132 \mu \mathrm{mol})$. After being stirred at room temperature for $1 \mathrm{~h}$, the reaction mixture was quenched with saturated aqueous $\mathrm{Na}_{2} \mathrm{SO}_{3}$ and aqueous $\mathrm{NH}_{4} \mathrm{Cl}$, and extracted with hexane/EtOAc (x2). The organic layer was washed with brine, and dried over $\mathrm{MgSO}_{4}$. Concentration and flash column chromatography (hexane/EtOAc 1:0-20:1) gave ketone 55 (35.5 mg, $31.3 \mu \mathrm{mol})$ in $80 \%$ yield: colorless oil; $[\alpha]_{\mathrm{D}}{ }^{18}=-8.3\left(c 0.81, \mathrm{CHCl}_{3}\right)$; IR (film) $v 2929,2865,1726,1463$, 1101, 1030, 817, $704 \mathrm{~cm}^{-1} ;{ }^{1} \mathrm{H}$ NMR (500 MHz, $\left.\mathrm{CDCl}_{3}\right) \delta 0.93(3 \mathrm{H}, \mathrm{d}, J=7.0 \mathrm{~Hz}, \mathrm{Me} 55), 0.99-1.09$ 
(42H, m, TBPS, TIPS, Me53, Me54, Me56, Me57), 1.58-1.74 (5H, m, H32, H35, H37, H47, H48), 1.82-2.09 (3H, m, H35, H36, H37), 2.07 (1H, ddd, $J=12.0,5.0,5.0 \mathrm{~Hz}, \mathrm{H} 32), 2.13-2.14$ (2H, m, H50x2), 2.37-2.44 (2H, m, H40, H43), 2.84 (1H, dd, $J=16.0,7.0 \mathrm{~Hz}, \mathrm{H} 40), 3.13$ (1H, ddd, $J=11.0$, 9.0, $5.0 \mathrm{~Hz}, \mathrm{H} 33), 3.23(1 \mathrm{H}, \mathrm{dd}, J=10.0,10.0 \mathrm{~Hz}, \mathrm{H} 46), 3.30-3.39$ (2H, m, H38, H34), 3.36 (3H, s, MOM), 3.54 (2H, m, H29, H42), 3.68 (1H, d, $J=10.5$ Hz, H29), 3.73 (1H, ddd, J = 7.0, 7.0, 7.0 Hz, H39), 3.92 (1H, dd, $J=9.0,4.5$ Hz, H52), 3.98-4.02 (3H, m, H44, H45, H52), 4.07 (1H, dd, $J=12.0$, $5.0 \mathrm{~Hz}, \mathrm{H} 31), 4.34$ (1H, m, H51), 4.58-4.65 (4H, m, NAPx3, MOM) 4.85 (1H, d, J = 6.5 Hz, MOM), $4.92(1 \mathrm{H}, \mathrm{d}, J=12.0 \mathrm{~Hz}, \mathrm{NAP}), 7.31-7.83(24 \mathrm{H}, \mathrm{m}, \mathrm{TBPS}, \mathrm{NAPx} 2) ;{ }^{13} \mathrm{C} \mathrm{NMR}\left(125 \mathrm{MHz}, \mathrm{CDCl}_{3}\right) \delta$ $12.1,12.5,12.7,13.7,15.9,18.1,19.3,26.8,28.7,36.4,37.6,39.8,41.9,42.6,45.5,47.4,56.2,67.3$, $68.4,71.1,71.4,73.1,73.5,74.0,77.8,78.7,80.5,81.0,82.2,83.5,84.6,98.7,108.6,125.6,125.8$, $125.9,126.0,126.1,126.2,127.2,127.3,127.65,127.68,127.82,127.84,128.1,129.3,132.8,132.9$, 133.26, 133.27, 133.34, 133.7, 134.3, 135.6, 135.7, 136.0, 136.7, 208.5; MALDI-TOF MS, calcd for $\mathrm{C}_{78} \mathrm{H}_{106} \mathrm{O}_{12} \mathrm{Si}_{2} \mathrm{Na}\left(\mathrm{M}+\mathrm{Na}^{+}\right)$1313.71, found 1313.72 .

Methylacetal 56. To a solution of ketone $55(95.5 \mathrm{mg}, 73.8 \mu \mathrm{mol})$ and $\mathrm{CH}(\mathrm{OMe})_{3}(67 \mu \mathrm{l})$ in hexane $(671 \mu \mathrm{l})$ at room temperature was added $\mathrm{TfOH}\left(0.25 \mathrm{M}\right.$ in $\left.\mathrm{CH}_{2} \mathrm{Cl}_{2}, 88.4 \mu \mathrm{l}, 22.1 \mu \mathrm{mol}\right)$. After being stirred for $1 \mathrm{~h}$, the reaction mixture was quenched with aqueous $\mathrm{NaHCO}_{3}$, and extracted with EtOAc (x3). The organic layer was washed with brine, and dried over $\mathrm{MgSO}_{4}$. Concentration and column chromatography gave methylacetal $56(57.7 \mathrm{mg}, 45.7 \mu \mathrm{mol})$ in $62 \%$ yield: colorless oil; $[\alpha]_{\mathrm{D}}{ }^{23}=-0.4(c$ $\left.1.15, \mathrm{CHCl}_{3}\right) ; \mathrm{IR}\left(\right.$ film) $\vee 2931,2866,1460,1092,816,704 \mathrm{~cm}^{-1} ;{ }^{1} \mathrm{H} \mathrm{NMR}\left(500 \mathrm{MHz}, \mathrm{CDCl}_{3}\right) \delta$ 1.02-1.08 (42H, m, Me53, Me54, Me55, Me57, TIPS, TBPS), 1.15 (3H, d, $J=6.0 \mathrm{~Hz}, \mathrm{Me} 56)$, 
1.47-1.73 (6H, m, H32, H35, H37, H40, H47, H48), 1.80-1.86 (3H, m, H35, H36, H37), 2.08 (1H, ddd, $J=12.0,5.0,5.0 \mathrm{~Hz}, \mathrm{H} 32), 2.13-2.19$ (3H, m, H43, H50x2), 2.45 (1H, dd, $J=13.5,4.0 \mathrm{~Hz}, \mathrm{H} 40), 2.90$ (1H, d, $J=9.0 \mathrm{~Hz}, \mathrm{H} 42), 3.00-3.12$ (2H, m, H33, H38), 3.22 (1H, m, H39), 3.30-3.36 (5H, m, H34, H44, MOM), 3.53 (1H, dd, $J=10.0,1.5 \mathrm{~Hz}, \mathrm{H} 45), 3.54(1 \mathrm{H}, \mathrm{d}, J=10.5 \mathrm{~Hz}, \mathrm{H} 29), 3.65(1 \mathrm{H}, \mathrm{dd}, J=$ 10.0, $10.0 \mathrm{~Hz}, \mathrm{H} 46), 3.67$ (1H, d, $J=10.5 \mathrm{~Hz}, \mathrm{H} 29), 3.88$ (1H, dd, $J=10.0,4.5 \mathrm{~Hz}, \mathrm{H} 52), 4.00$ (1H, dd, $J=10.0,2.5 \mathrm{~Hz}, \mathrm{H} 52), 4.05(1 \mathrm{H}, \mathrm{dd}, J=12.0,5.0 \mathrm{~Hz}, \mathrm{H} 31), 4.30(1 \mathrm{H}, \mathrm{m}, \mathrm{H} 51), 4.59(1 \mathrm{H}, \mathrm{d}, J=12.0$ Hz, NAP), $4.61(1 \mathrm{H}, \mathrm{d}, J=12.0 \mathrm{~Hz}, \mathrm{NAP}), 4.74(1 \mathrm{H}, \mathrm{d}, J=12.0 \mathrm{~Hz}, \mathrm{NAP}), 4.81(1 \mathrm{H}, \mathrm{d}, J=12.0 \mathrm{~Hz}$, NAP), 7.30-7.83 (24H, m, TBPS, NAPx2); $\left.{ }^{13} \mathrm{C} \mathrm{NMR} \mathrm{(125} \mathrm{MHz,} \mathrm{CDCl}{ }_{3}\right) \delta 12.4,12.7,13.6,16.8,18.1$, $19.3,20.3,26.8,27.3,28.3,37.0,38.1,38.4,40.0,40.5,42.3,48.4,67.4,68.4,68.8,71.1,71.5,72.4$, $72.8,73.4,77.7,78.5,81.4,81.9,83.6,84.2,85.3,99.1,108.4,125.4,125.6,125.7,125.8,125.9,126.1$, $126.2,127.2,127.3,127.5,127.6,127.7,127.8,128.1,129.30,129.34,132.7,132.9,133.2,132.3,133.6$, 134.3, 135.6, 136.1, 137.3; MALDI-TOF MS, calcd for $\mathrm{C}_{77} \mathrm{H}_{104} \mathrm{O}_{11} \mathrm{Si}_{2} \mathrm{Na} 1283.70\left(\mathrm{M}+\mathrm{Na}^{+}\right)$, found 1283.70.

HIJKLM ring fragment 57. To a solution of methylacetal 56 (57.7 mg, $45.7 \mu \mathrm{mol}), \mathrm{Et}_{3} \mathrm{SiH}(11.1 \mu \mathrm{l}$, 91.4 $\mu \mathrm{mol})$ and powdered MS4A in $\mathrm{CH}_{2} \mathrm{Cl}_{2}$ at $-50{ }^{\circ} \mathrm{C}$ was added $\mathrm{BF}_{3} \cdot \mathrm{OEt}_{2}\left(0.02 \mathrm{M}\right.$ solution in $\mathrm{CH}_{2} \mathrm{Cl}_{2}$, $3.5 \mu \mathrm{l}, 68.6 \mu \mathrm{mol})$. The reaction mixture was allowed to warm to $-20{ }^{\circ} \mathrm{C}$ for $1 \mathrm{~h}$, and quenched with $1 \% \mathrm{Et}_{3} \mathrm{~N}$-hexane and aqueous $\mathrm{NaHCO}_{3}$. The mixture was extracted with EtOAc (x3), and the organic layer was washed with brine, and dried over $\mathrm{MgSO}_{4}$. Concentration and column chromatography (hexane/EtOAc 25:1-5:1), and purified by HPLC (YMC-Pack SIL-06, 10x250 mm, UV $254 \mathrm{~nm}$, hexane/EtOAc 9:1, $5.0 \mathrm{~mL} / \mathrm{min})$ gave HIJKLM ring fragment $57\left(\mathrm{t}_{\mathrm{R}}=8.4 \mathrm{~min}, 44.4 \mathrm{mg}, 36.0 \mu \mathrm{mol}\right)$ in 
78\% yield: colorless oil; $[\alpha]_{\mathrm{D}}{ }^{21}=-4.3\left(c 1.32, \mathrm{CHCl}_{3}\right)$; IR (film) $\vee 2930,1462,1101,817 \mathrm{~cm}^{-1} ;{ }^{1} \mathrm{H}$ NMR (500 MHz, $\mathrm{CDCl}_{3}$ ) $\delta$ 1.02-1.18 (45H, m, TBPS, TIPS, Me53, Me54, Me55, Me56, Me57), 1.56-1.73 (5H, m, H35, H37, H40, H47, H48), 1.70 (1H, ddd, $J=12.0,12.0,12.0 \mathrm{~Hz}, \mathrm{H} 32), 1.80-1.87(3 \mathrm{H}, \mathrm{m}$, H35, H36, H37), 2.09 (1H, ddd, $J=12.0,5.0,5.0$ Hz, H32), 2.14-2.25 (4H, m, H40, H43, H50x2), 2.86 (1H, dd, $J=9.0,4.5 \mathrm{~Hz}, \mathrm{H} 42), 2.99$ (1H, m, H38), 3.06-3.17 (2H, m, H33, H39), 3.33 (1H, ddd, $J=$ 11.0, 9.0, 3.0 Hz, H34), 3.40-3.44 (2H, m, H44, H46), $3.53(1 \mathrm{H}, \mathrm{d}, J=10.5 \mathrm{~Hz}, \mathrm{H} 29), 3.62(1 \mathrm{H}, \mathrm{d}, J=$ $9.5 \mathrm{~Hz}, \mathrm{H} 45), 3.67(1 \mathrm{H}, \mathrm{d}, J=10.5 \mathrm{~Hz}, \mathrm{H} 29), 3.82(1 \mathrm{H}, \mathrm{dd}, J=10.5,5.5 \mathrm{~Hz}, \mathrm{H} 52), 3.86(1 \mathrm{H}, \mathrm{ddd}, J=$ 11.0, 9.0, $5.0 \mathrm{~Hz}, \mathrm{H} 41), 3.98(1 \mathrm{H}, \mathrm{dd}, J=10.5,2.0 \mathrm{~Hz}, \mathrm{H} 52), 4.05$ (1H, dd, $J=12.0,5.0 \mathrm{~Hz}, \mathrm{H} 31), 4.27$ $(1 \mathrm{H}, \mathrm{m}, \mathrm{H} 51), 4.59(1 \mathrm{H}, \mathrm{d}, J=12.0 \mathrm{~Hz}, \mathrm{NAP}), 4.62(1 \mathrm{H}, \mathrm{d}, J=12.0 \mathrm{~Hz}, \mathrm{NAP}), 4.74(1 \mathrm{H}, \mathrm{d}, J=12.5 \mathrm{~Hz}$, NAP), $4.81(1 \mathrm{H}, \mathrm{d}, J=12.5 \mathrm{~Hz}, \mathrm{NAP}), 7.32-7.83(24 \mathrm{H}, \mathrm{m}, \mathrm{NAPx} 2, \mathrm{TBPS}) ;{ }^{13} \mathrm{C}$ NMR $(125 \mathrm{MHz}$, $\left.\mathrm{CDCl}_{3}\right) \delta 12.5,12.7,13.5,15.9,18.22,18.24,19.3,19.9,26.8,27.5,28.2,38.1,38.4,40.1,40.6,41.6$, $42.5,46.5,67.3,68.4,71.1,71.4,71.9,72.1,73.4,74.2,77.6,77.8,78.5,81.0,83.1,83.8,84.6,86.7$, $108.9,125.6,125.8,125.9,126.0,126.12,126.14,126.2,127.2,127.3,127.6,127.7,127.8,128.1$, 129.31, 129.32, 132.8, 132.9, 133.1, 133.2, 133.7, 134.3, 135.6, 135.7, 136.1, 136.8; MALDI-TOF MS, calcd for $\mathrm{C}_{76} \mathrm{H}_{102} \mathrm{O}_{10} \mathrm{Si}_{2} \mathrm{Na}\left(\mathrm{M}+\mathrm{Na}^{+}\right)$1253.69, found 1253.69.

Alcohol 58. To HIJKLM segment $57(25.6 \mathrm{mg}, 20.7 \mu \mathrm{mol})$ in a plastic test tube was added buffered pyridinium hydrofluroide $[1 \mathrm{~mL}$, a mixture of THF $(1 \mathrm{~mL})$, pyridine $(0.57 \mathrm{~mL})$ and pyridinium hydrofluoride $(0.19 \mathrm{~mL})]$. After being stirred at room temperature for $12 \mathrm{~h}$, the reaction mixture was quenched with saturated aqueous $\mathrm{CuSO}_{4}$. The mixture was extracted with EtOAc (x3), and the organic layer was washed with saturated aqueous $\mathrm{NaHCO}_{3}$, brine, and dried over $\mathrm{MgSO}_{4}$. Concentration and 
column chromatography (hexane/EtOAc 10:1) gave alcohol 58 (15.7 mg, $15.8 \mu \mathrm{mol})$ in $76 \%$ yield: colorless oil; $[\alpha]_{\mathrm{D}}{ }^{20}=-2.1\left(c 0.99, \mathrm{CHCl}_{3}\right) ; \mathrm{IR}(\mathrm{film}) \vee 2927,2866,1734,1458,1093,1030 \mathrm{~cm}^{-1} ;{ }^{1} \mathrm{H}$ NMR (500 MHz, $\mathrm{CDCl}_{3}$ ) $\delta$ 1.03-1.11 (36H, m, TIPS, Me53, Me54, Me55, Me56, Me57), 1.50-1.77 (7H, m, H32, H35x2, H37, H40, H47, H48), 1.84-1.86 (2H, m, H36, H37), 2.04-2.24 (5H, m, H32, H40, H43, H50x2), 2.85 (1H, dd, $J=9.0,5.0 \mathrm{~Hz}, \mathrm{H} 42), 2.98(1 \mathrm{H}, \mathrm{ddd}, J=9.5,9.5,3.0 \mathrm{~Hz}, \mathrm{H} 38)$, 3.04-3.12 (2H, m, H33, H39), 3.35 (1H, ddd, $J=9.5,9.5,3.5 \mathrm{~Hz}, \mathrm{H} 34), 3.40-3.43$ (3H, m, H29, H44, H46), $3.55(1 \mathrm{H}, \mathrm{d}, J=11.0 \mathrm{~Hz}, \mathrm{H} 29), 3.62(1 \mathrm{H}, \mathrm{d}, J=10.0 \mathrm{~Hz}, \mathrm{H} 45), 3.82(1 \mathrm{H}, \mathrm{dd}, J=10.0,5.0 \mathrm{~Hz}$, H52), $3.87(1 \mathrm{H}, \mathrm{m}, \mathrm{H} 41), 3.90(1 \mathrm{H}, \mathrm{dd}, J=11.5,4.5 \mathrm{~Hz}, \mathrm{H} 31), 3.98(1 \mathrm{H}, \mathrm{dd}, J=10.0,1.5 \mathrm{~Hz}, \mathrm{H} 52)$, $4.26(1 \mathrm{H}, \mathrm{m}, \mathrm{H} 51), 4.58(1 \mathrm{H}, \mathrm{d}, J=13.0 \mathrm{~Hz}, \mathrm{NAP}), 4.62(1 \mathrm{H}, \mathrm{d}, J=13.0 \mathrm{~Hz}, \mathrm{NAP}), 4.74(1 \mathrm{H}, \mathrm{d}, J=$ $12.0 \mathrm{~Hz}, \mathrm{NAP}), 4.80(1 \mathrm{H}, J=12.0 \mathrm{~Hz}, \mathrm{NAP}), 7.43-7.83(14 \mathrm{H}, \mathrm{m}, \mathrm{NAPx}) ;{ }^{13} \mathrm{C}$ NMR $(125 \mathrm{MHz}$, $\left.\mathrm{CDCl}_{3}\right) \delta 12.7,12.9,13.5,15.9,18.0,18.1,19.9,24.7,27.5,28.0,29.0,29.2,29.3,29.4,29.6,33.4,37.8$, $38.4,40.1,40.5,41.6,42.5,45.4,46.1,67.4,71.1,71.4,71.9,72.1,73.3,74.1,77.7,78.5,80.9,83.2$, $83.3,84.6,86.7,108.9,125.6,125.7,125.8,125.9,126.0,126.1,126.2,127.6,127.70,127.75,127.83$, 128.1, 132.8, 132.9, 133.1, 133.2, 135.6, 136.8; MALDI-TOF MS, calcd for $\mathrm{C}_{60} \mathrm{H}_{84} \mathrm{O}_{10} \mathrm{SiNa}\left(\mathrm{M}+\mathrm{Na}^{+}\right)$ 1015.57, found 1015.57.

Homoallylic alcohol 60. To a solution of DMSO $(277 \mu \mathrm{l}, 3.9 \mathrm{mmol})$ and $(\mathrm{COCl})_{2}(173 \mu \mathrm{l}, 1.9 \mathrm{mmol})$ in $\mathrm{CH}_{2} \mathrm{Cl}_{2}(3.8 \mathrm{~mL})$ at $-70{ }^{\circ} \mathrm{C}$ was added alcohol $58(24.3 \mathrm{mg}, 24.4 \mu \mathrm{mol})$ in $\mathrm{CH}_{2} \mathrm{Cl}_{2}(0.9 \mathrm{~mL})$. After being stirred at the same temperature for $30 \mathrm{~min}$, the mixture was treated with $\mathrm{Et}_{3} \mathrm{~N}(1.1 \mathrm{~mL}, 7.8 \mathrm{mmol})$ was added, and allowed to warm to $-40{ }^{\circ} \mathrm{C}$ over $1 \mathrm{~h}$. Then the reaction was quenched with aqueous $\mathrm{NH}_{4} \mathrm{Cl}$. The mixture was extracted with $\mathrm{Et}_{2} \mathrm{O}$ (x2), and the organic layer was washed with saturated 
aqueous $\mathrm{NH}_{4} \mathrm{Cl}$ and brine, dried over $\mathrm{MgSO}_{4}$, and concentrated. The residue was purified through a pad of silica gel to give aldehyde 59, which was used in the next reaction without further purification. $\mathrm{MgBr}_{2}$ was prepared by stirring $\mathrm{Mg}$ turnings (59 mg, $2.4 \mathrm{mmol}$ ) and dibromoethane (420 $\left.\mu \mathrm{l}, 4.9 \mathrm{mmol}\right)$ in $\mathrm{Et}_{2} \mathrm{O}(2.0 \mathrm{~mL})$ for $30 \mathrm{~min}$ at $0{ }^{\circ} \mathrm{C}$ and following evaporation. To the solid of $\mathrm{MgBr}_{2} \mathrm{Was}$ added the aldehyde 59 in $\mathrm{CH}_{2} \mathrm{Cl}_{2}(3.8 \mathrm{~mL})$ and the resulting mixture was cooled to $-78{ }^{\circ} \mathrm{C}$. The mixture was then treated with allyltributyltin $(152 \mu \mathrm{l}, 488 \mu \mathrm{mol})$, allowed to warm to $8{ }^{\circ} \mathrm{C}$ over $8 \mathrm{~h}$, and then quenched with saturated aqueous $\mathrm{NH}_{4} \mathrm{Cl}$. The mixture was extracted with hexane/EtOAc (x2), and the organic layer was washed with brine, and dried over $\mathrm{Na}_{2} \mathrm{SO}_{4}$. Concentration and flash column chromatography (hexane/EtOAc 30:1-10:1) gave the desired homoallylic alcohol 60 (17.4 mg, 16.8 $\mu \mathrm{mol})$ in $69 \%$ yield over 2 steps and C29-epimer $(3.7 \mathrm{mg}, 3.5 \mu \mathrm{mol})$ in $14 \%$ yield over 2 steps. 60: colorless oil; $[\alpha]_{\mathrm{D}}{ }^{28}=0.7\left(c 0.91, \mathrm{CHCl}_{3}\right)$; IR (film) $v 2942,2866,1458,1090,1030 \mathrm{~cm}^{-1} ;{ }^{1} \mathrm{H} \mathrm{NMR}(500$ $\left.\mathrm{MHz}, \mathrm{CDCl}_{3}\right) \delta$ 1.02-1.14 (36H, m, TIPS, Me53, Me54, Me55, Me56, Me57), 1.28-1.64 (5H, m, H35, H37, H40, H47, H48), 1.69 (1H, ddd, $J=12.0,12.0,12.0 \mathrm{~Hz}, \mathrm{H} 32), 1.75(1 \mathrm{H}, \mathrm{dd}, J=14.0,3.0 \mathrm{~Hz}$, H35), 1.82-1.87 (2H, m, H36, H37), $2.00(1 \mathrm{H}, \mathrm{d}, J=10.0 \mathrm{~Hz}, \mathrm{OH} 29), 2.08$ (1H, ddd, $J=12.0,5.0,5.0$ Hz, H32), 2.14-2.24 (5H, m, H28, H40, H43, H50x2), 2.35 (1H, m, H28), 2.85 (1H, dd, J = 9.5, 5.0 Hz, H42), 2.98 (1H, ddd, $J=10.0,10.0,3.0$ Hz, H38), 3.03-3.11 (2H, m, H33, H39), 3.30 (1H, ddd, $J=9.5$, 9.5, 3.0 Hz, H34), $3.42(1 \mathrm{H}, \mathrm{dd}, J=10.0,10.0 \mathrm{~Hz}, \mathrm{H} 46), 3.43(1 \mathrm{H}, \mathrm{d}, J=4.0 \mathrm{~Hz}, \mathrm{H} 44), 3.60(1 \mathrm{H}, \mathrm{dt}, J=$ 10.0, 10.0, 3.0 Hz, H29), $3.62(1 \mathrm{H}, \mathrm{d}, J=10.0 \mathrm{~Hz}, \mathrm{H} 45), 3.82(1 \mathrm{H}, \mathrm{dd}, J=9.5,5.0 \mathrm{~Hz}, \mathrm{H} 52), 3.86(1 \mathrm{H}$, ddd, $J=11.5,9.5,5.0 \mathrm{~Hz}, \mathrm{H} 41), 3.98(1 \mathrm{H}, \mathrm{dd}, J=9.5,1.5 \mathrm{~Hz}, \mathrm{H} 52), 4.16(1 \mathrm{H}, \mathrm{dd}, J=12.0,5.0 \mathrm{~Hz}$, H31), 4.27 (1H, m, H51), $4.58(1 \mathrm{H}, \mathrm{d}, J=12.0 \mathrm{~Hz}, \mathrm{NAP}), 4.62(1 \mathrm{H}, \mathrm{d}, J=12.0 \mathrm{~Hz}, \mathrm{NAP}), 4.74(1 \mathrm{H}, \mathrm{d}$, $J=12.0 \mathrm{~Hz}, \mathrm{NAP}), 4.80(1 \mathrm{H}, \mathrm{d}, J=12.0 \mathrm{~Hz}, \mathrm{NAP}), 5.06(1 \mathrm{H}, \mathrm{d}, J=10.0,2.0 \mathrm{~Hz}, \mathrm{H} 26), 5.08(1 \mathrm{H}, \mathrm{dd}, J$ 
$=17.0,2.0 \mathrm{~Hz}, \mathrm{H} 26), 5.92(1 \mathrm{H}, \mathrm{ddt}, J=17.0,10.0,7.0 \mathrm{~Hz}, \mathrm{H} 27), 7.43-7.83(14 \mathrm{H}, \mathrm{m}, \mathrm{NAPx} 2) ;{ }^{13} \mathrm{C}$

NMR (125 MHz, $\left.\mathrm{CDCl}_{3}\right)$ 12.8, 13.5, 13.6, 15.9, 18.1, 18.2, 19.9, 27.5, 28.0, 29.6, 35.7, 38.0, 38.5, 40.1, $40.5,41.6,42.5,45.4,45.9,67.6,71.1,71.4,71.9,72.1,72.7,73.2,74.1,77.8,78.1,78.5,80.9,83.0$ $83.1,84.6,86.7,108.9,116.4,125.65,125.68,125.8,125.9,126.0,126.1,126.2,127.70,127.75,127.84$, 128.1, 132.8, 132.9, 133.2, 133.3, 135.6, 136.5, 136.8; MALDI-TOF MS, calcd for $\mathrm{C}_{63} \mathrm{H}_{88} \mathrm{O}_{10} \mathrm{SiNa}$ $\left(\mathrm{M}+\mathrm{Na}^{+}\right)$1055.60, found 1055.60.

Right wing 14 of 51-hydroxyCTX3C. To a solution of homoallylic alcohol $60(5.7 \mathrm{mg}, 5.51 \mu \mathrm{mol})$ in THF/DMF (3:1, $0.8 \mathrm{~mL})$ at $0{ }^{\circ} \mathrm{C}$ were added $\mathrm{NaH}(13.2 \mathrm{mg}, 551 \mu \mathrm{mol}), \mathrm{NAPBr}(60.9 \mathrm{mg}, 275.5 \mu \mathrm{mol})$ and TBAI $(20.3 \mathrm{mg}, 55.1 \mu \mathrm{mol})$. After being stirred at room temperature for $1 \mathrm{~d}$, the reaction mixture was quenched with saturated aqueous $\mathrm{NH}_{4} \mathrm{Cl}$, and extracted with EtOAc (x2). The organic layer was washed with brine, and dried over $\mathrm{MgSO}_{4}$. Concentration and column chromatography (hexane/EtOAc 1:1) gave NAP ether 14, which was further purified by HPLC (YMC Pack Sil-06, 6.0x250 mm, UV $254 \mathrm{~nm}$, hexane/EtOAc 1:0-2:8, $1.5 \mathrm{~mL} / \mathrm{min})$ to give right wing $14(5.9 \mathrm{mg}, 5.03$ $\mu \mathrm{mol})$ in $91 \%$ yield: colorless oil; $[\alpha]_{\mathrm{D}}^{22}=2.3\left(c 0.39, \mathrm{CHCl}_{3}\right) ; \mathrm{IR}($ film $) \vee 2943,1459,1090,1031,815$ $736 \mathrm{~cm}^{-1} ;{ }^{1} \mathrm{H}$ NMR $\left(500 \mathrm{MHz}, \mathrm{CDCl}_{3}\right) \delta$ 1.01-1.11 (33H, m, TIPS, Me54, Me55, Me56, Me57), 1.14 (3H, s, Me53), 1.37 (1H, ddd, $J=11.5,11.5,11.5 \mathrm{~Hz}, \mathrm{H} 40), 1.50-1.63$ (3H, m, H35, H37, H47), 1.72 (1H, ddd, $J=11.5,11.5,11.5 \mathrm{~Hz}, \mathrm{H} 32), 1.80-1.89$ (4H, m, H35, H36, H37, H48), 2.10 (1H, ddd, $J=$ 11.5, 5.5, 5.5 Hz, H40), 2.13-2.22 (4H, m, H32, H43, H50x2), 2.49-2.58 (2H, m, H28x2), 2.85 (1H, dd, $J=9.5,4.5 \mathrm{~Hz}, \mathrm{H} 42), 2.99$ (1H, ddd, $J=11.5,9.5,2.0 \mathrm{~Hz}, \mathrm{H} 38), 3.04-3.12(2 \mathrm{H}, \mathrm{m}, \mathrm{H} 33, \mathrm{H} 39), 3.30$ $(1 \mathrm{H}, \mathrm{ddd}, J=9.5,9.5,3.5 \mathrm{~Hz}, \mathrm{H} 34), 3.42(1 \mathrm{H}, \mathrm{dd}, J=9.5,9.5 \mathrm{~Hz}, \mathrm{H} 46), 3.43(1 \mathrm{H}, \mathrm{d}, J=3.5 \mathrm{~Hz}, \mathrm{H} 44)$, 
$3.62(1 \mathrm{H}, \mathrm{d}, J=9.5 \mathrm{~Hz}, \mathrm{H} 45), 3.66(1 \mathrm{H}, \mathrm{dd}, J=8.0,4.5 \mathrm{~Hz}, \mathrm{H} 29), 3.82(1 \mathrm{H}, \mathrm{dd}, J=9.5,5.0 \mathrm{~Hz}, \mathrm{H} 52)$, $3.85(1 \mathrm{H}, \mathrm{ddd}, J=11.5,9.5,5.5 \mathrm{~Hz}, \mathrm{H} 41), 3.98(1 \mathrm{H}, \mathrm{dd}, J=9.5,1.5 \mathrm{~Hz}, \mathrm{H} 52), 4.26(1 \mathrm{H}, \mathrm{dd}, J=11.5$, $5.0 \mathrm{~Hz}, \mathrm{H} 31), 4.27$ (1H, m, H51), 4.59 (1H, d, $J=12.0 \mathrm{~Hz}, \mathrm{NAP}), 4.62$ (1H, d, $J=12.0 \mathrm{~Hz}, \mathrm{NAP}), 4.74$ $(1 \mathrm{H}, \mathrm{d}, J=12.5 \mathrm{~Hz}, \mathrm{NAP}), 4.75(1 \mathrm{H}, \mathrm{d}, J=12.5 \mathrm{~Hz}, \mathrm{NAP}), 4.81(1 \mathrm{H}, \mathrm{d}, J=11.0 \mathrm{~Hz}, \mathrm{NAP}), 4.84(1 \mathrm{H}, \mathrm{d}$, $J=11.0 \mathrm{~Hz}, \mathrm{NAP}), 5.01(1 \mathrm{H}, \mathrm{dd}, J=10.5,2.0 \mathrm{~Hz}, \mathrm{H} 26), 5.12(1 \mathrm{H}, \mathrm{dd}, J=17.0,2.0 \mathrm{~Hz}, \mathrm{H} 26), 5.99(1 \mathrm{H}$, ddt, $J=17.0,10.5,6.5 \mathrm{~Hz}, \mathrm{H} 27), 7.41-7.83(21 \mathrm{H}, \mathrm{m}, \mathrm{NAPx} 3) ;{ }^{13} \mathrm{C} \mathrm{NMR}\left(125 \mathrm{MHz}, \mathrm{CDCl}_{3}\right) \delta 13.3$, $13.8,14.2,14.3,16.2,18.57,18.59,20.1,27.8,28.3,29.6,29.9,31.8,32.1,33.5,38.6,38.7,40.4,40.8$ $41.8,42.7,45.7,46.2,68.6,71.4,71.7,71.8,72.1,72.4,73.7,74.4,78.0,78.8,80.2,81.1,82.2,83.2$ $83.3,84.8,86.9,109.2,116.1,125.6,125.90,125.93,126.01,126.03,126.05,126.12,126.2,126.32$, $126.38,126.39,126.5,127.8,127.94,127.97,128.00,128.07,128.09,128.4,132.9,133.1,133.2,133.4$ 133.5, 135.9, 137.0, 137.3, 137.5; HRMS (FAB), calcd for $\mathrm{C}_{74} \mathrm{H}_{96} \mathrm{O}_{10} \mathrm{SiNa}\left(\mathrm{M}+\mathrm{Na}^{+}\right)$1195.6670, found 1195.6653. 


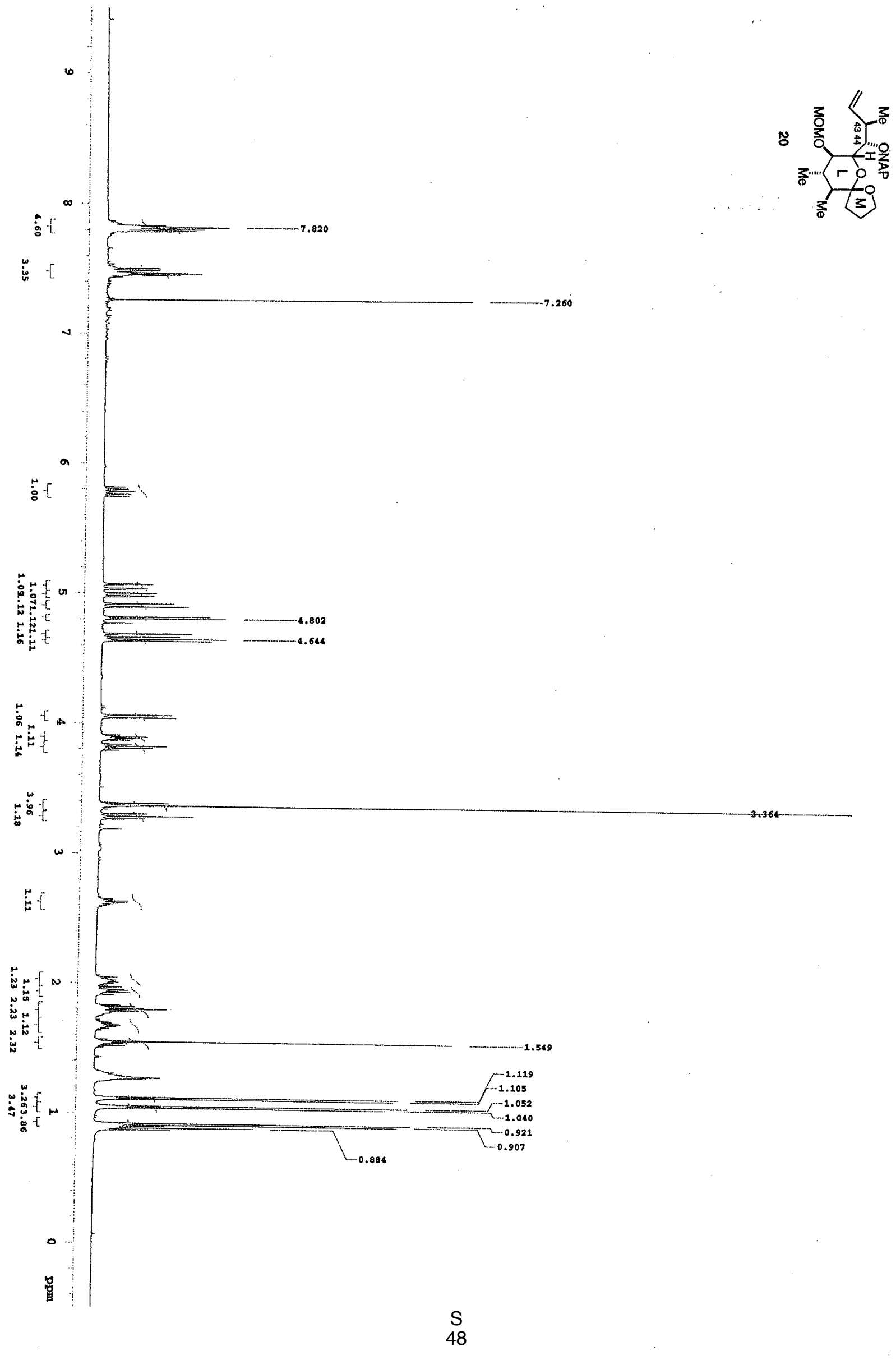




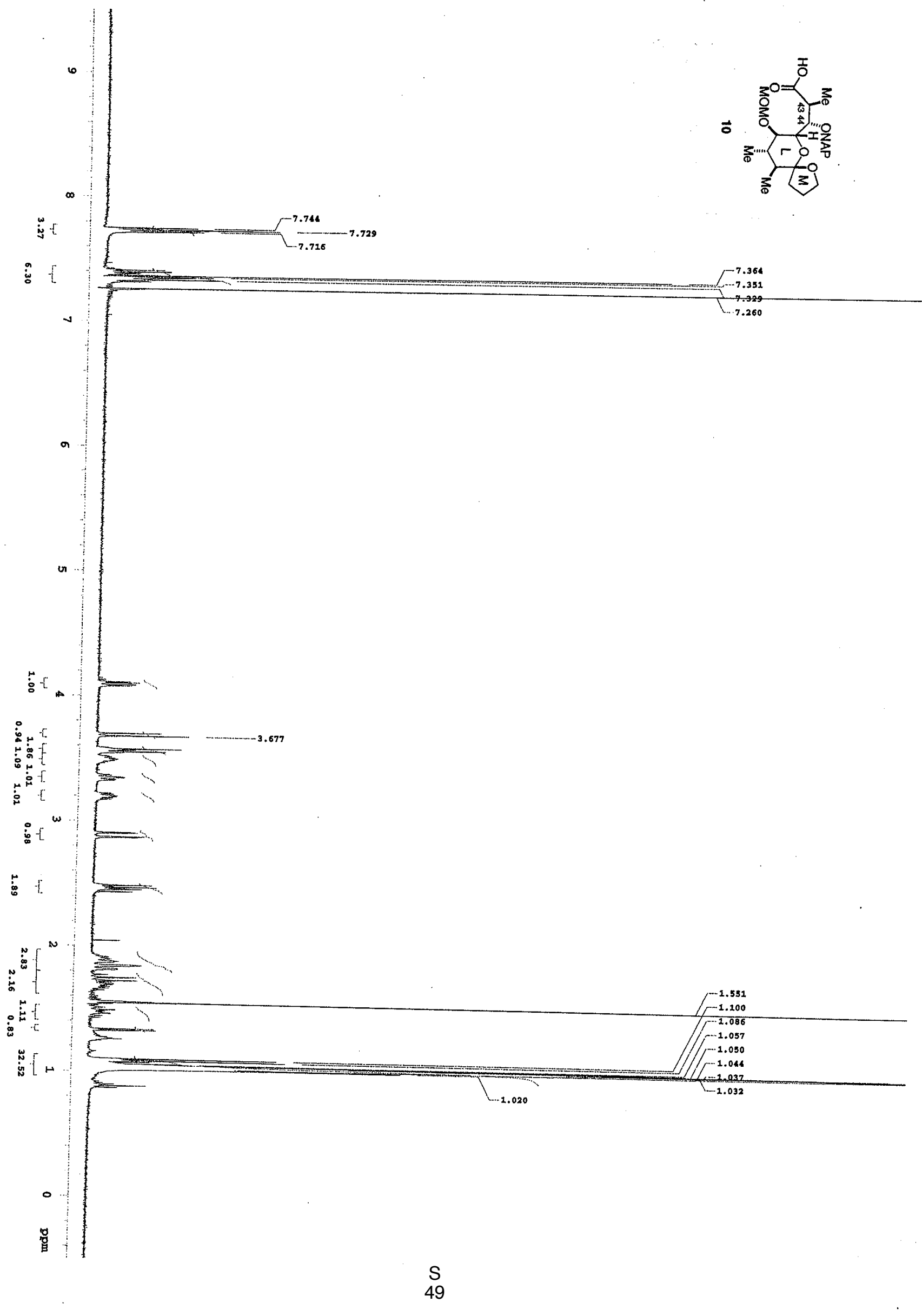




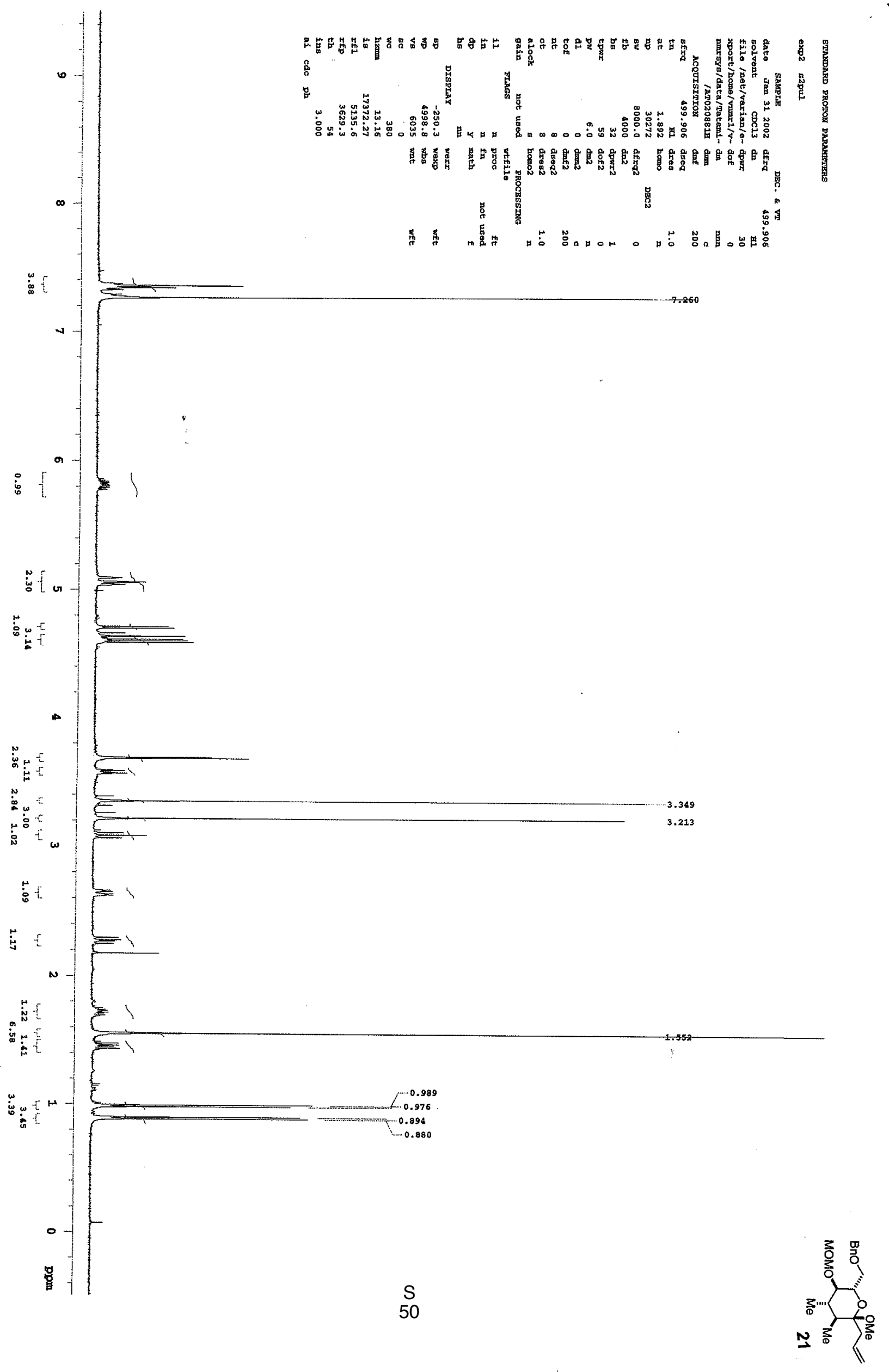




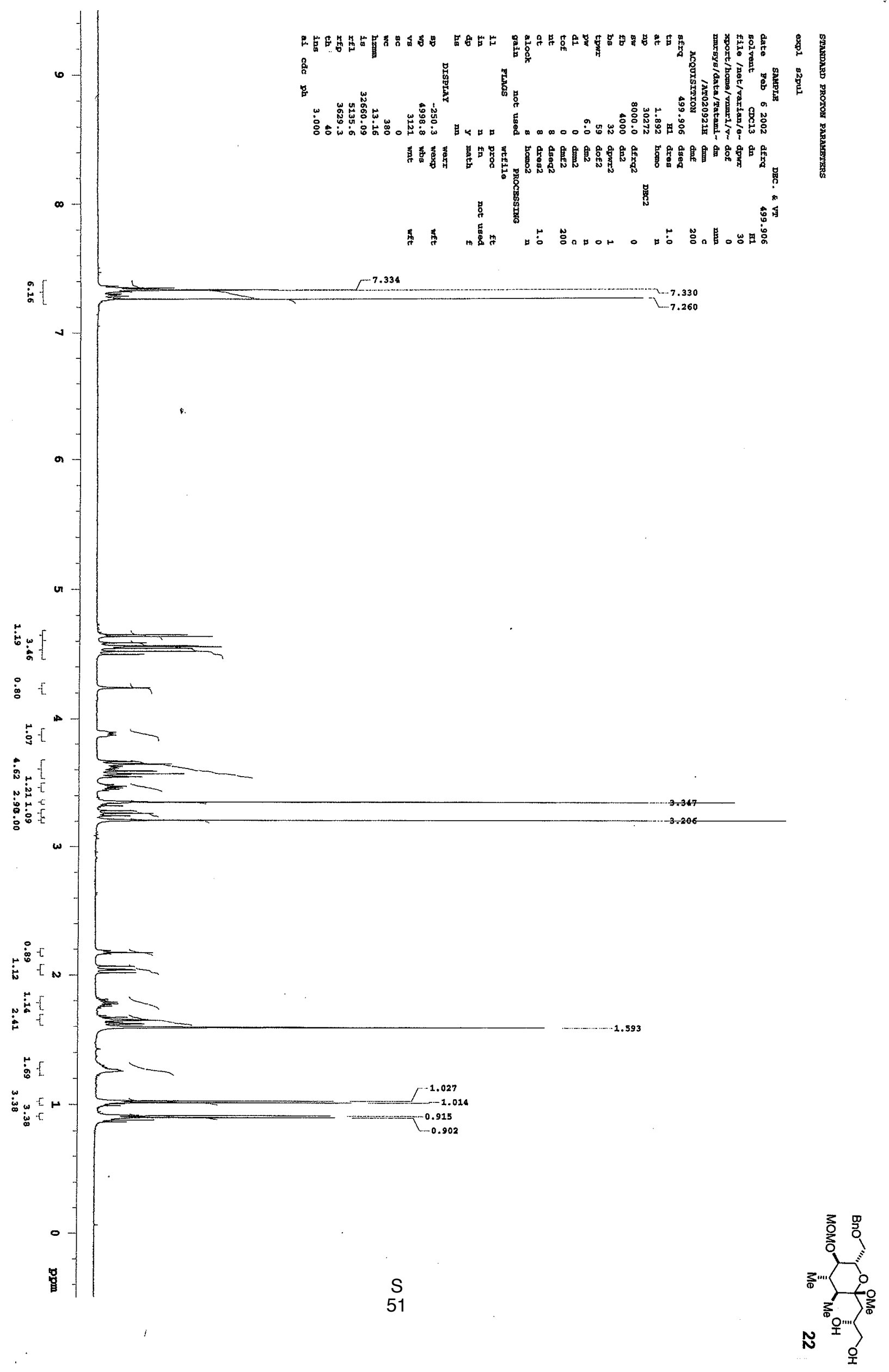




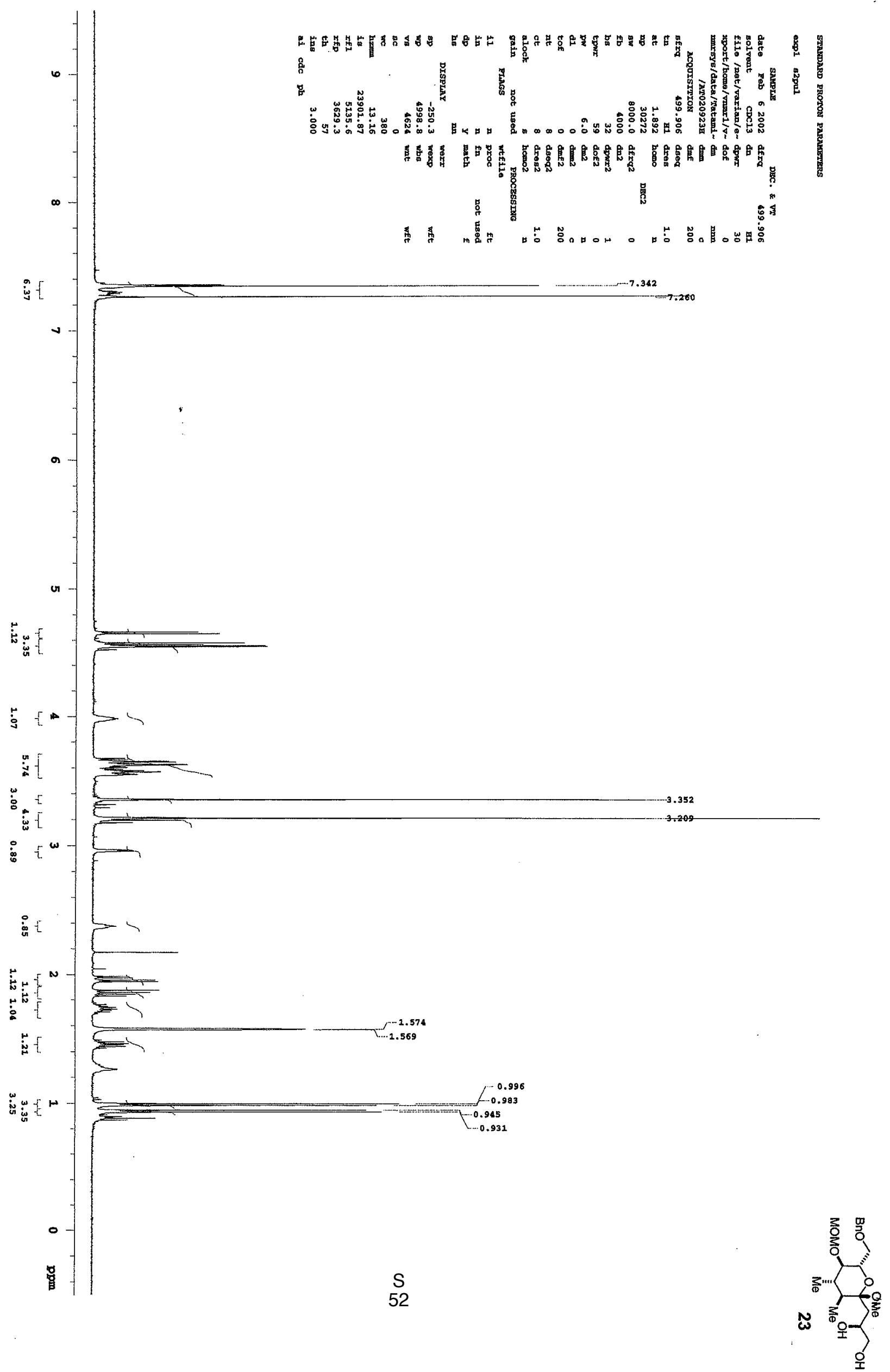



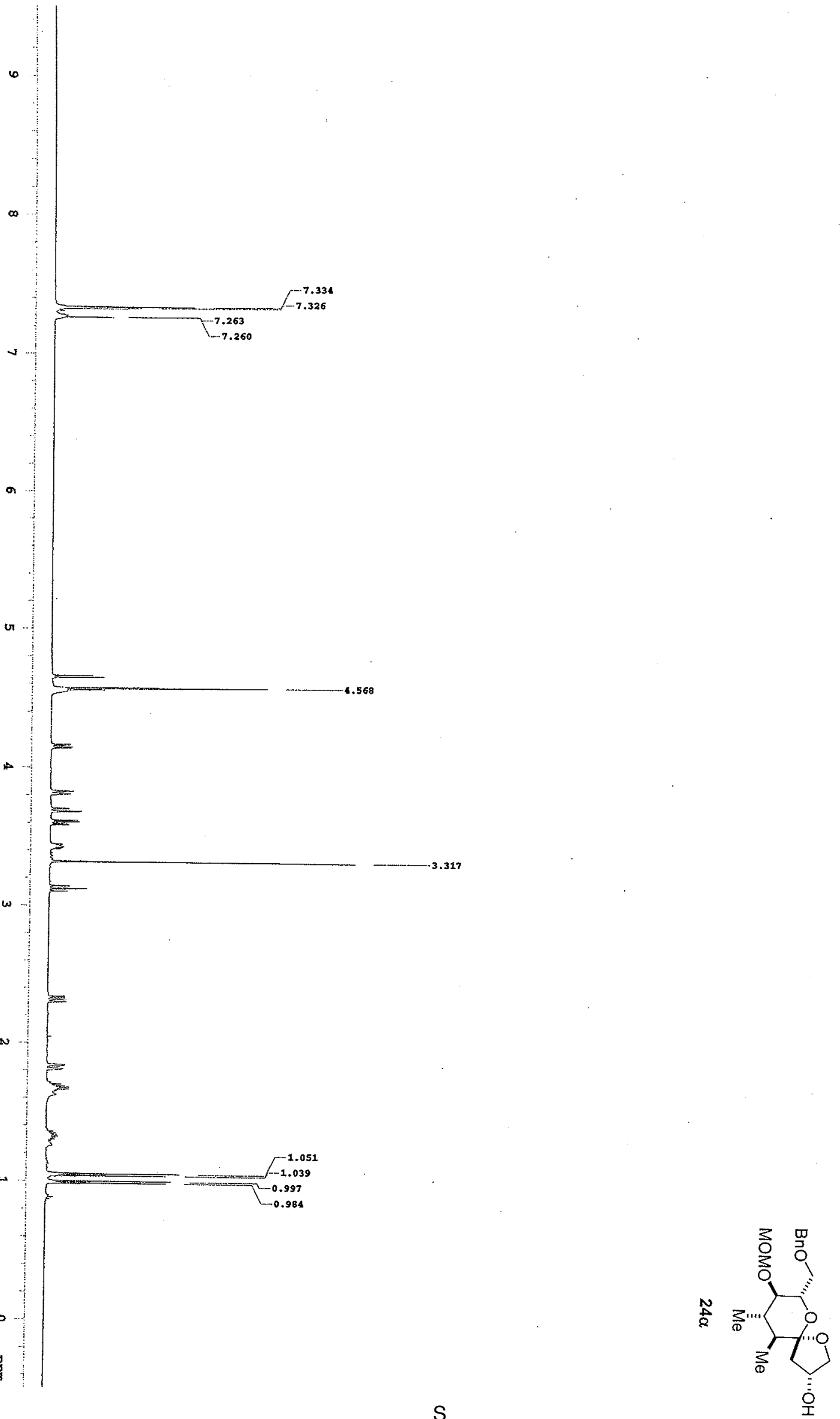
$-7.338$
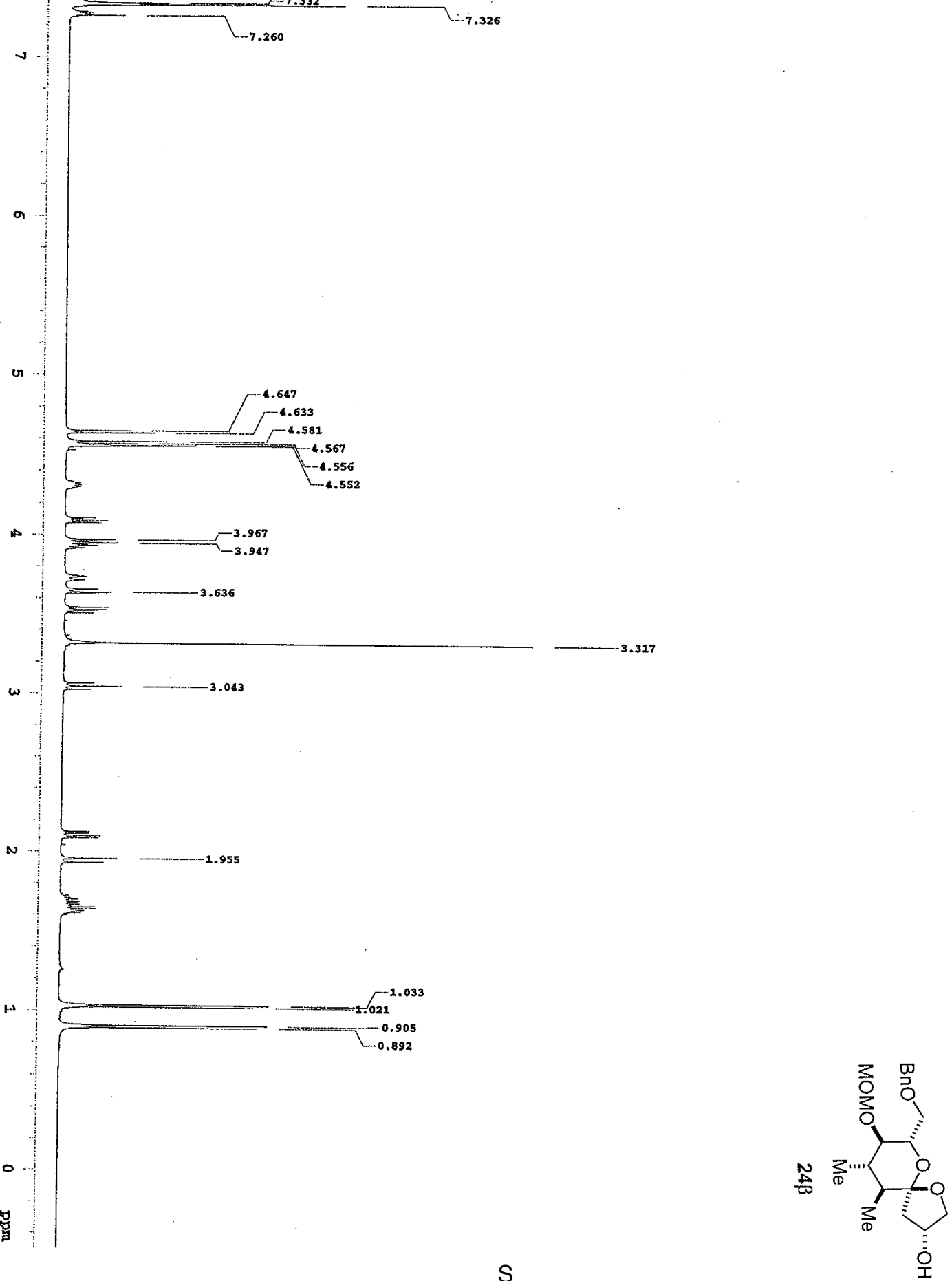


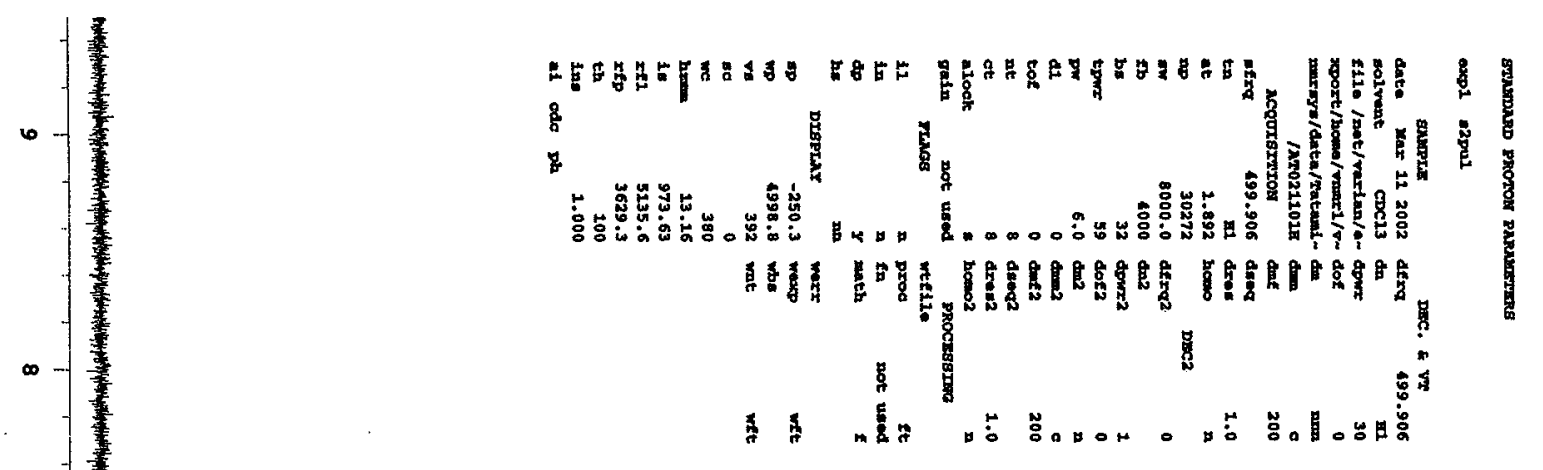

a

u

$\dot{8}$
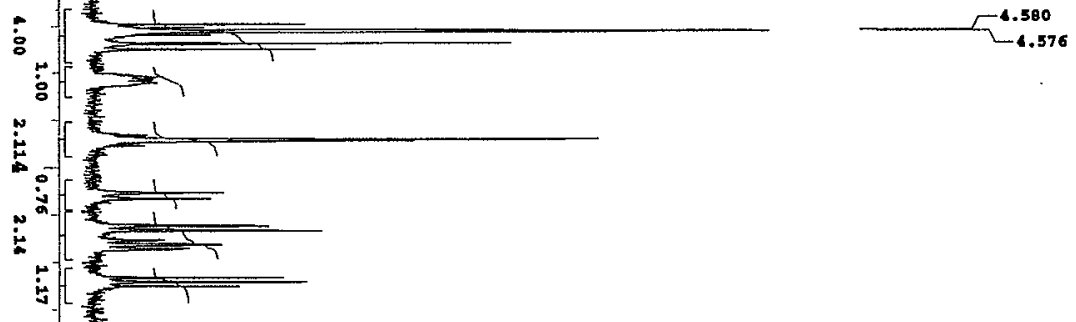

is

$\omega$

:

s

.

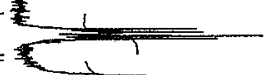

亳
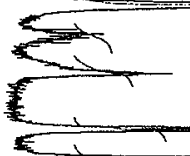

$\Gamma^{1.03}$

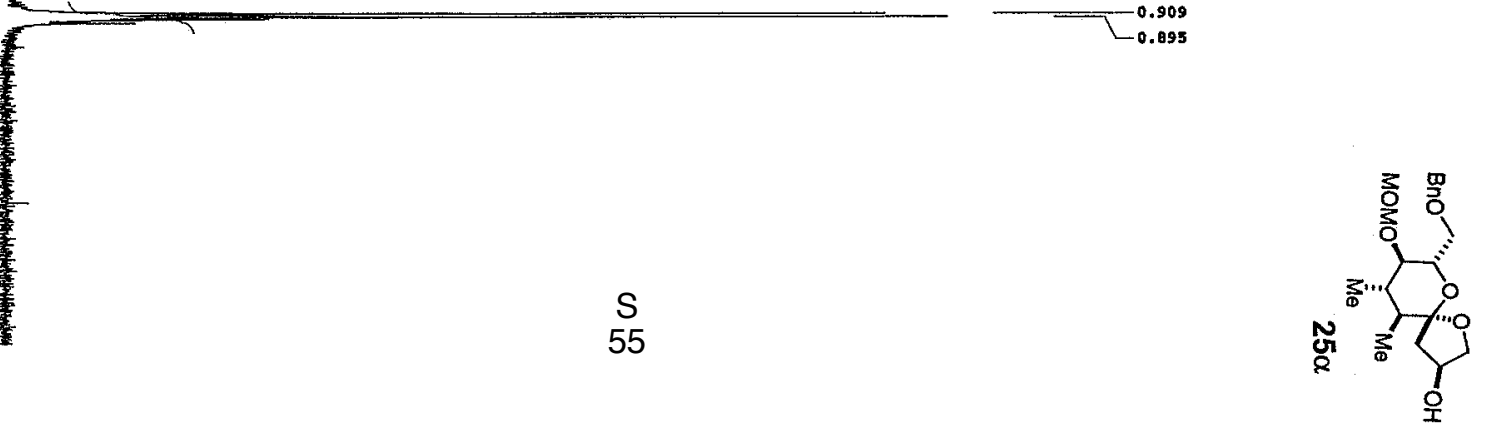




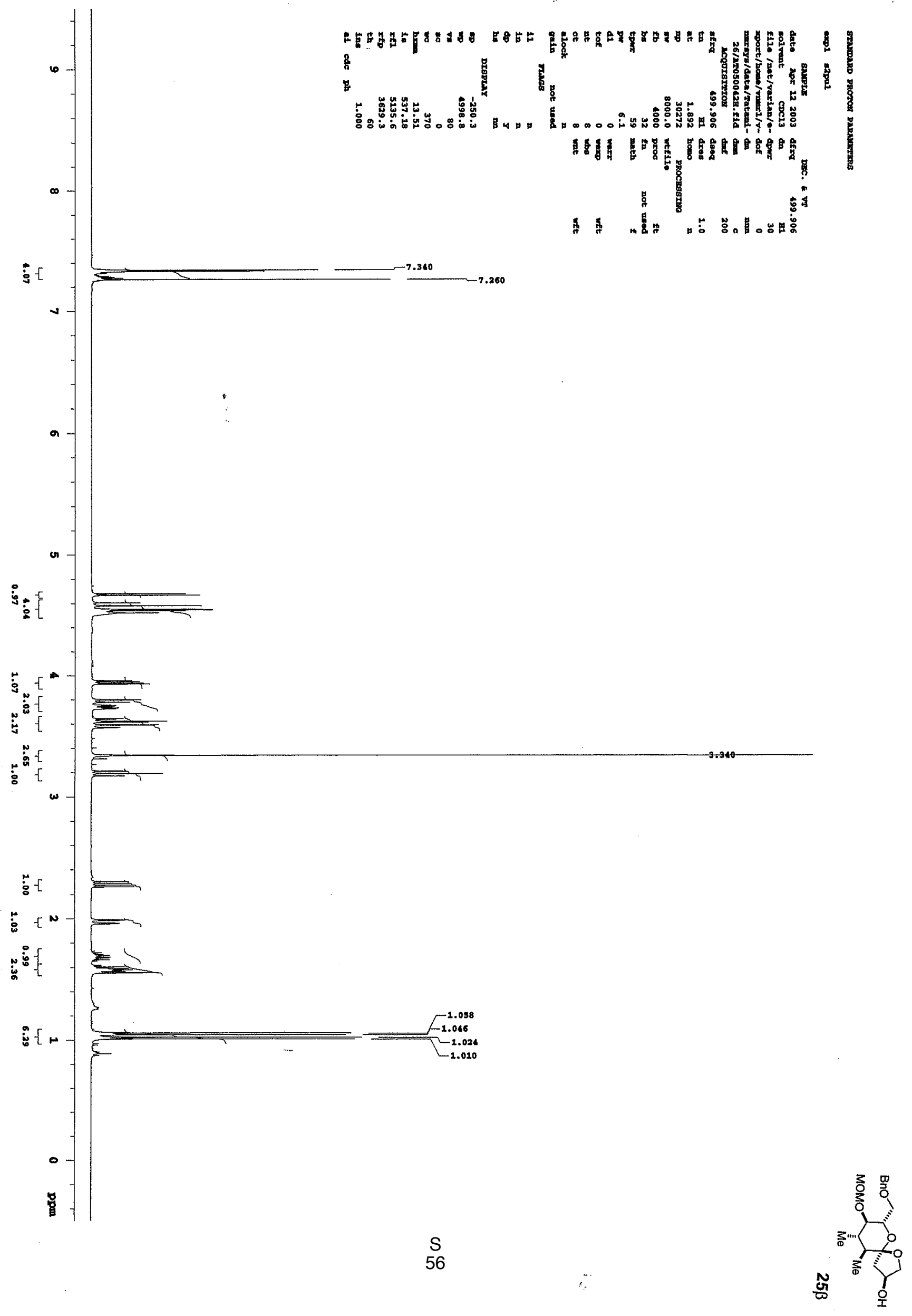




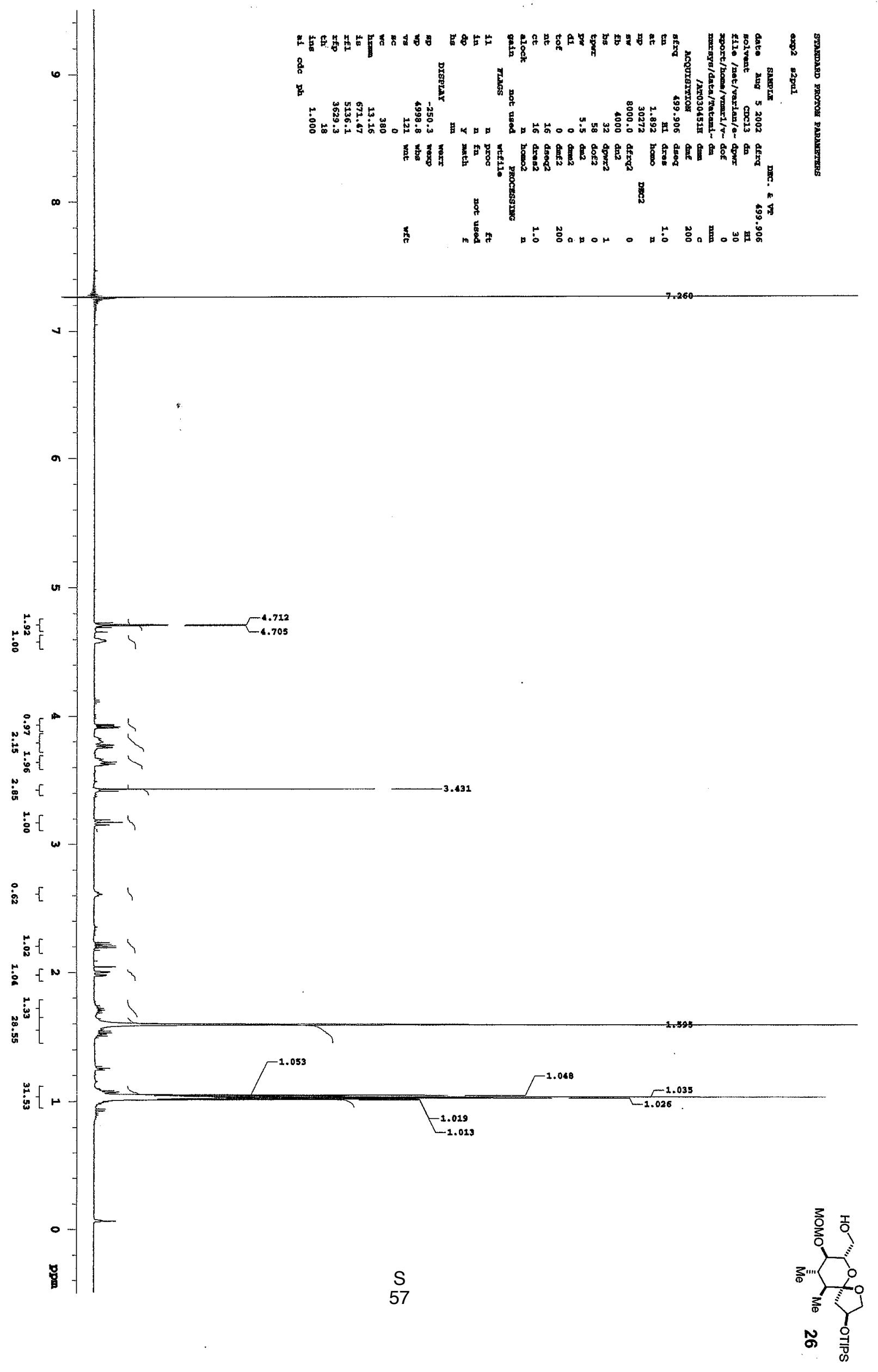




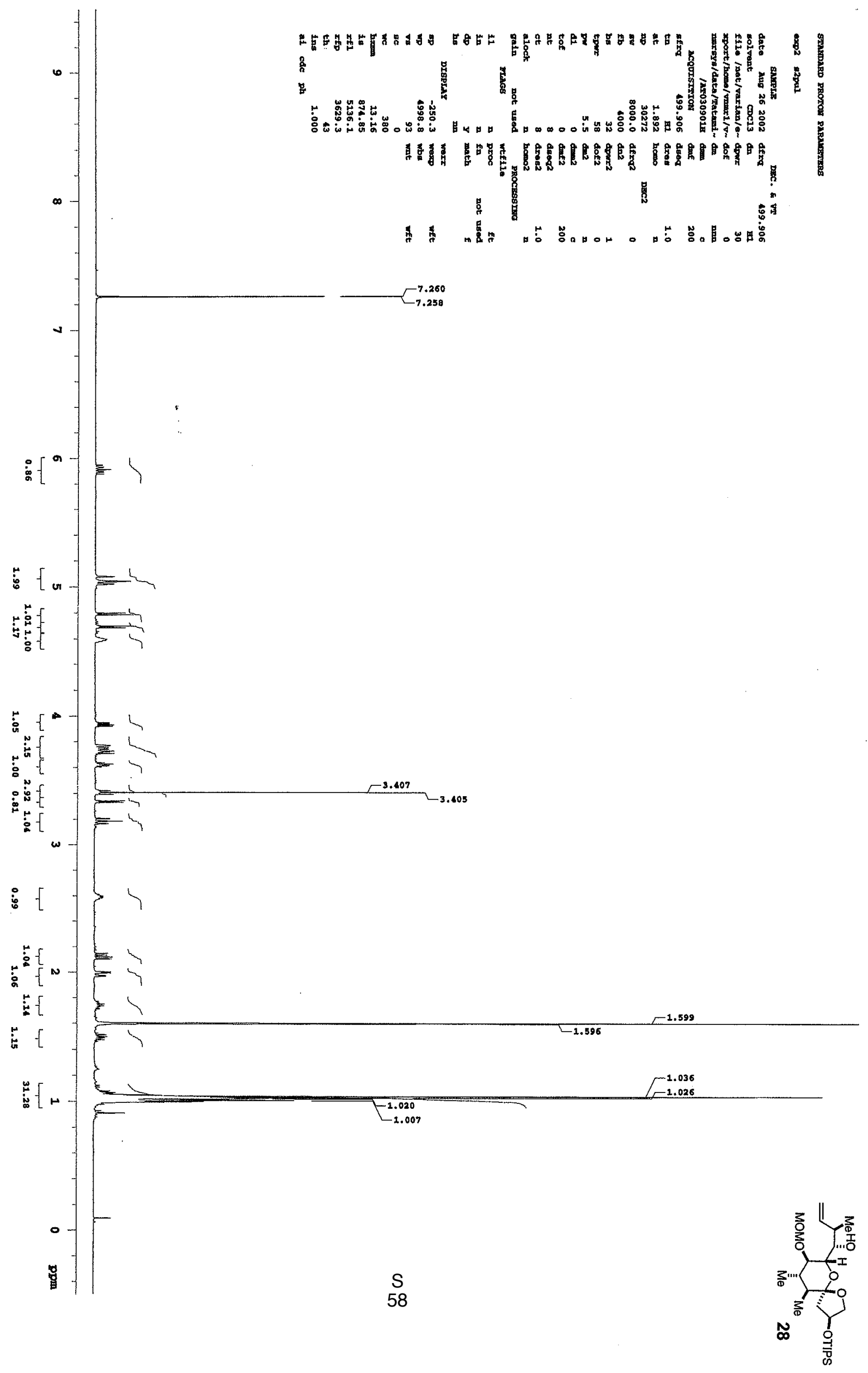




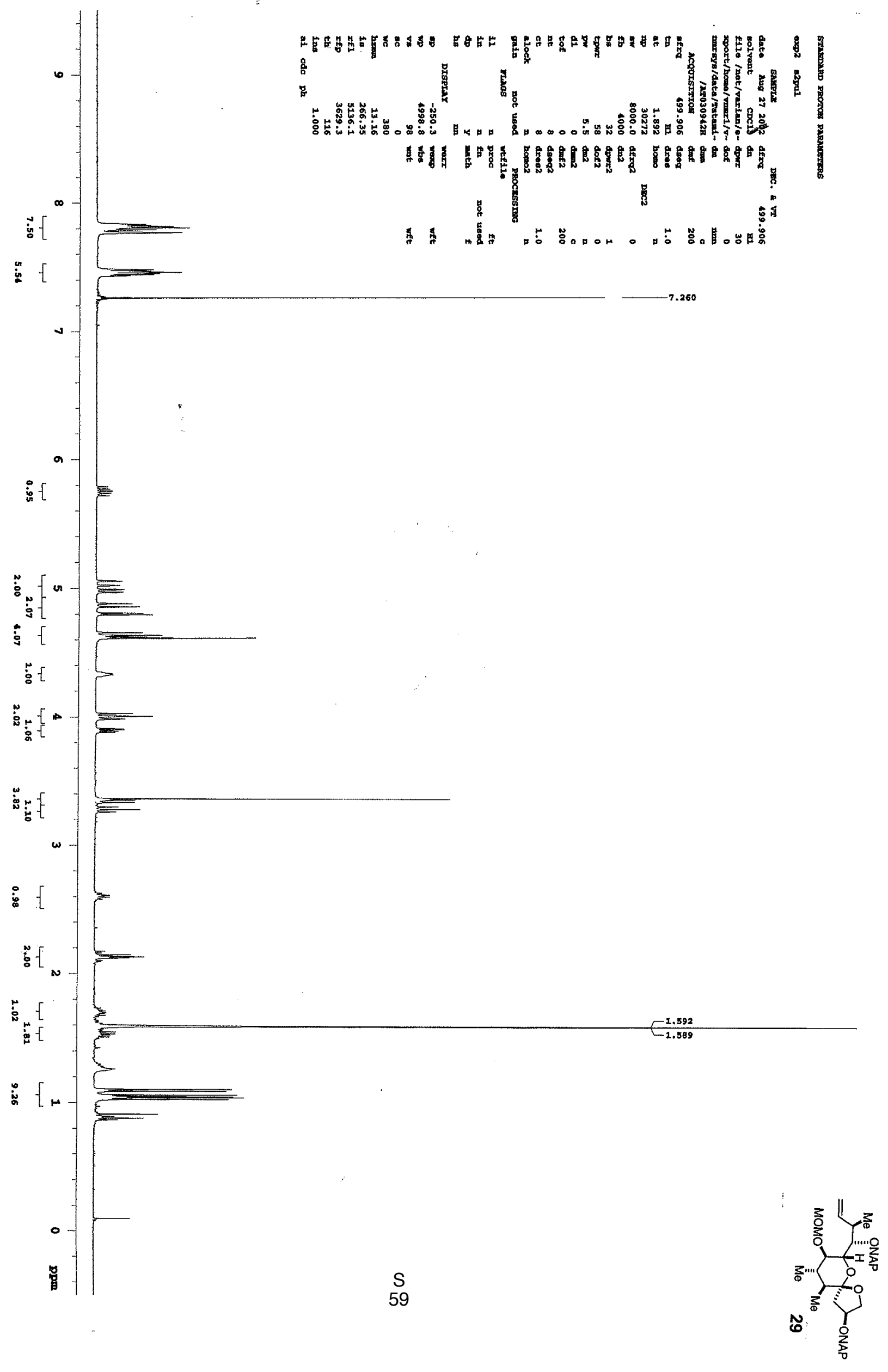




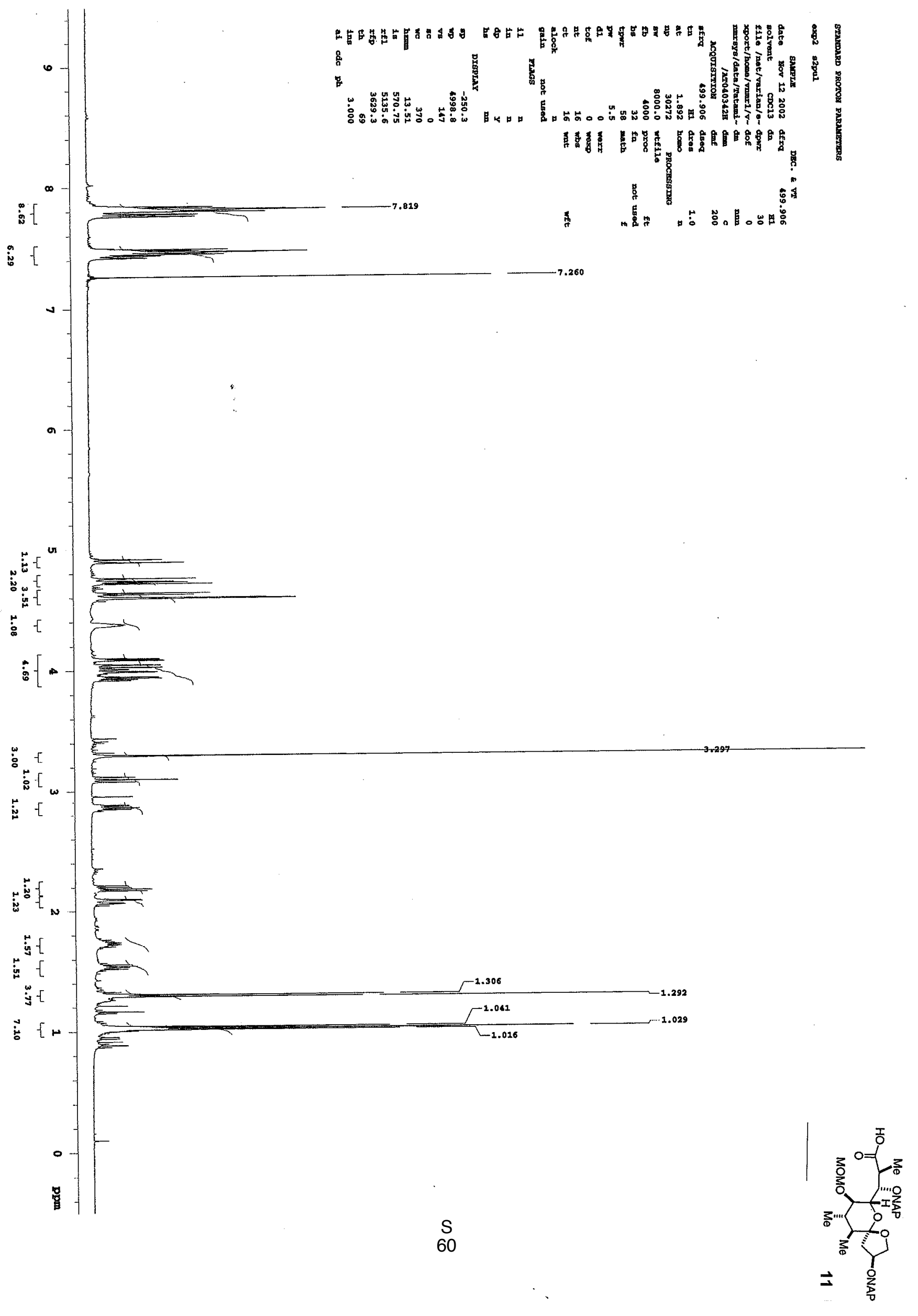




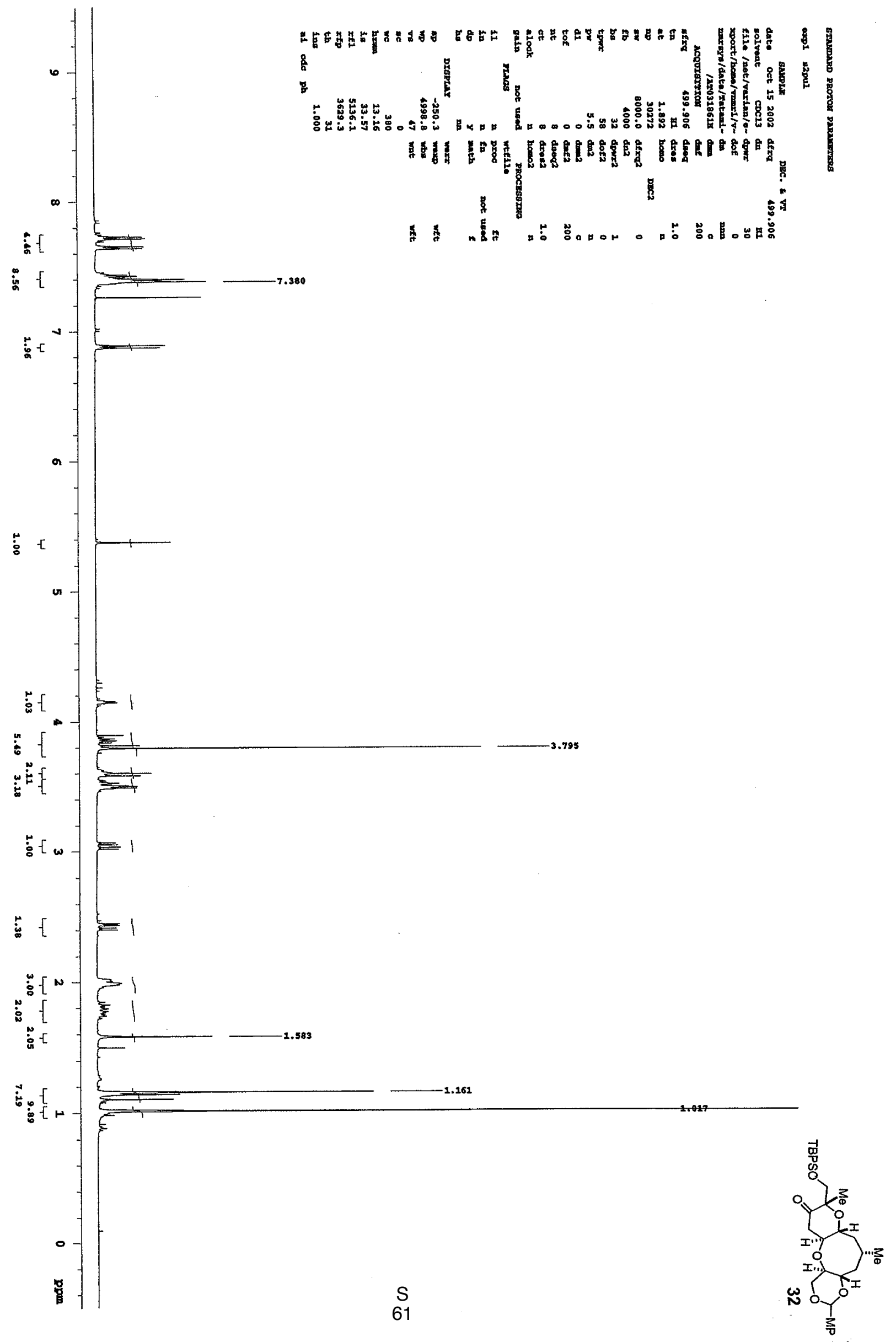




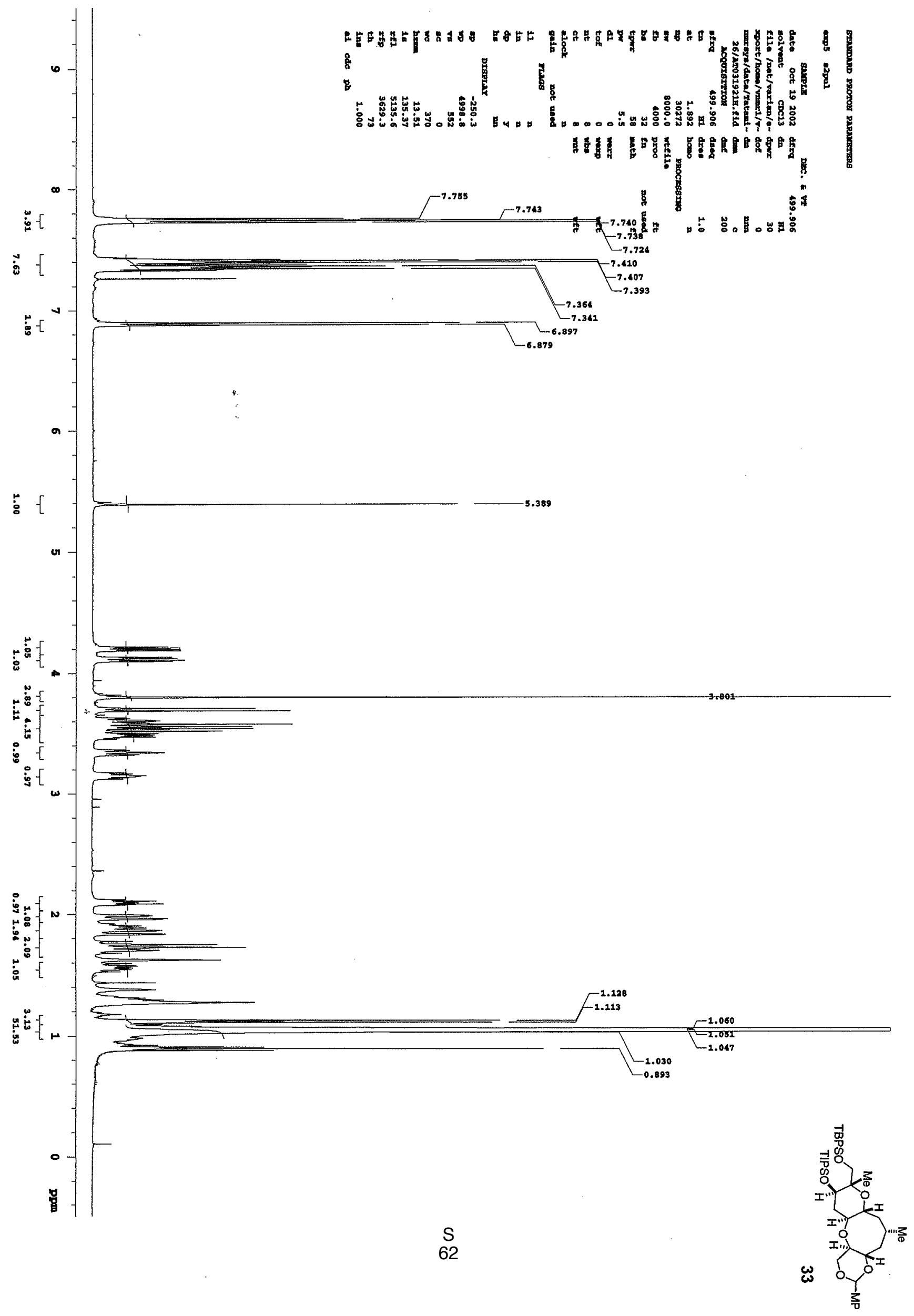




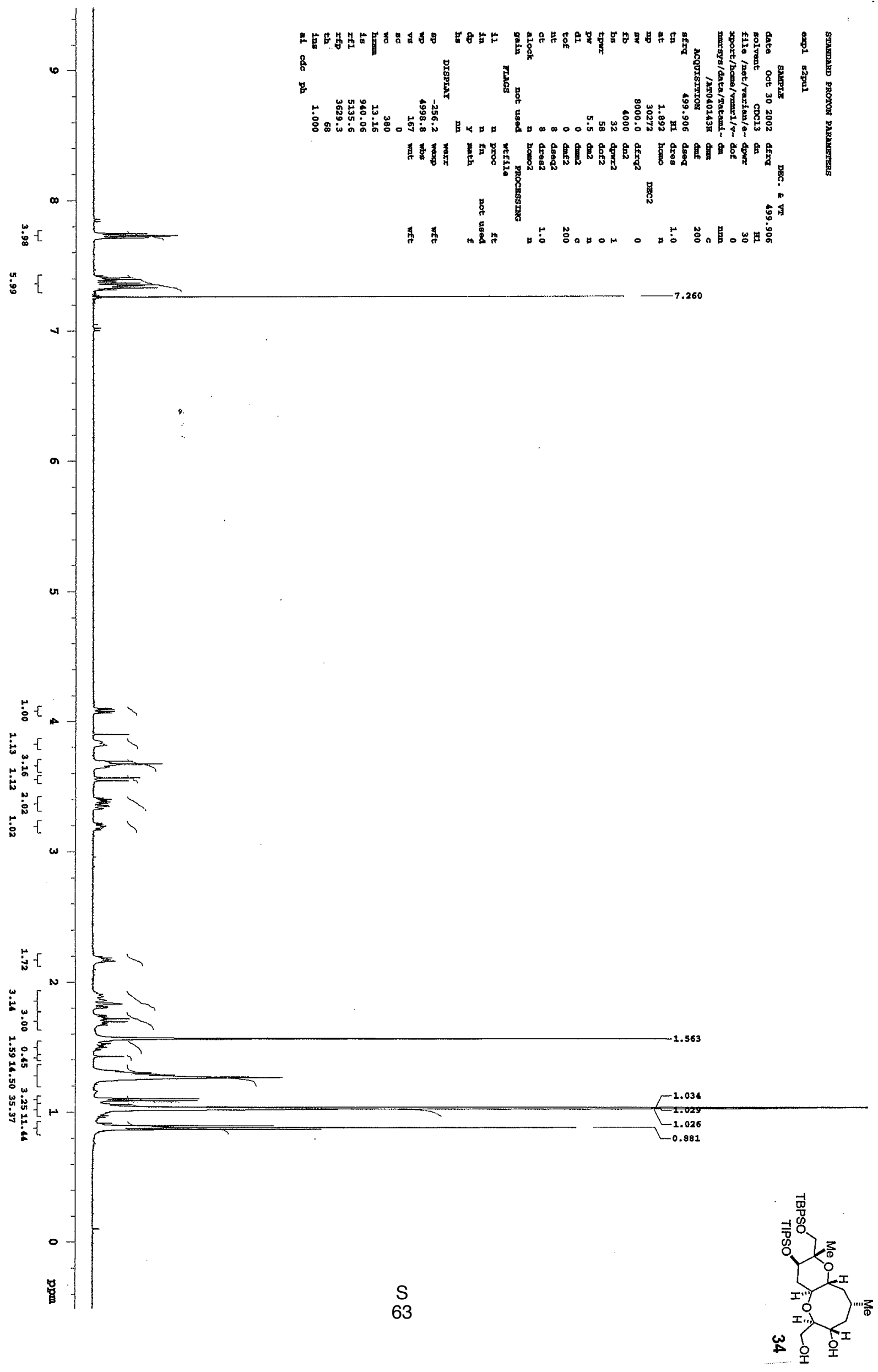




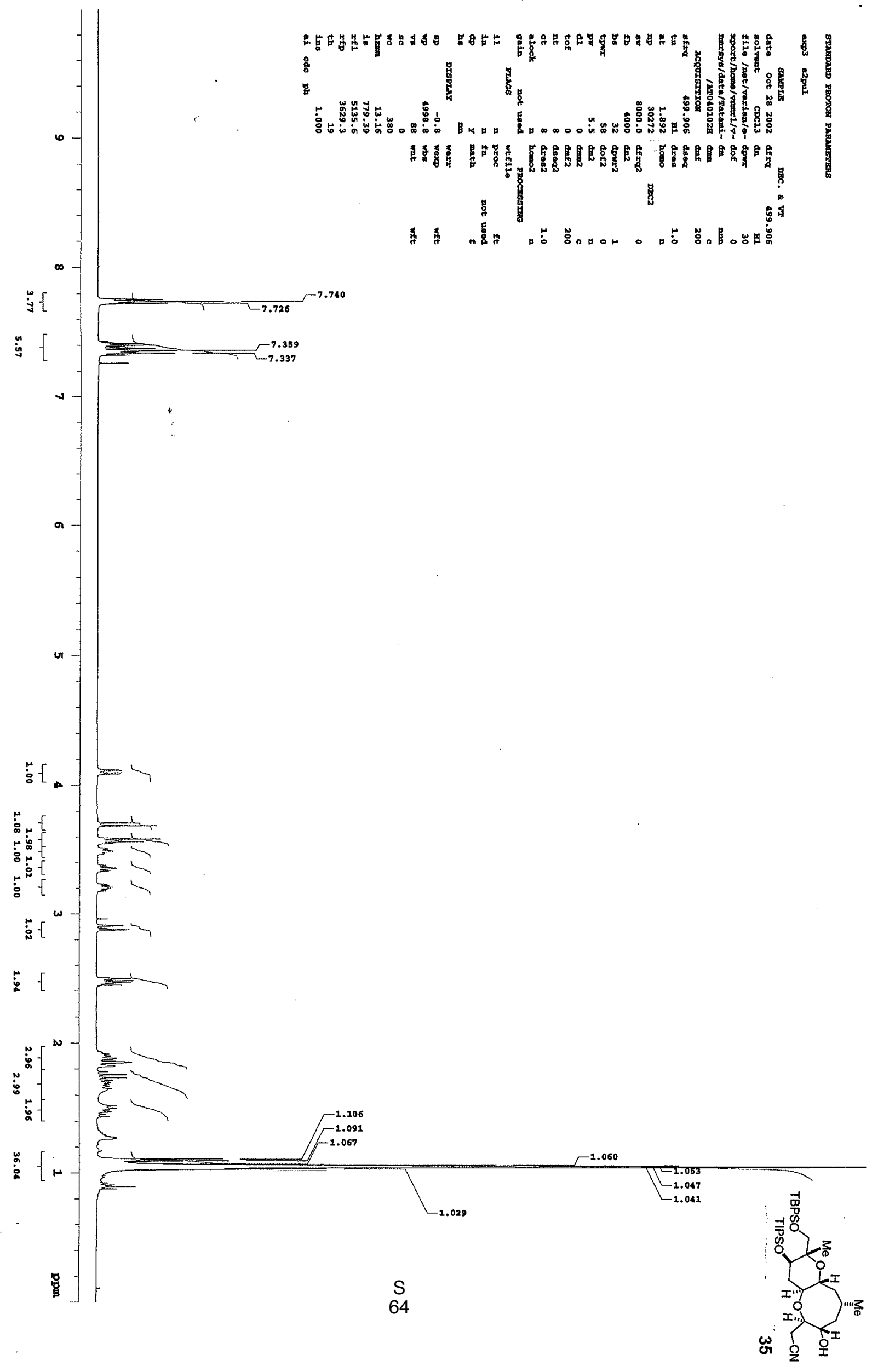




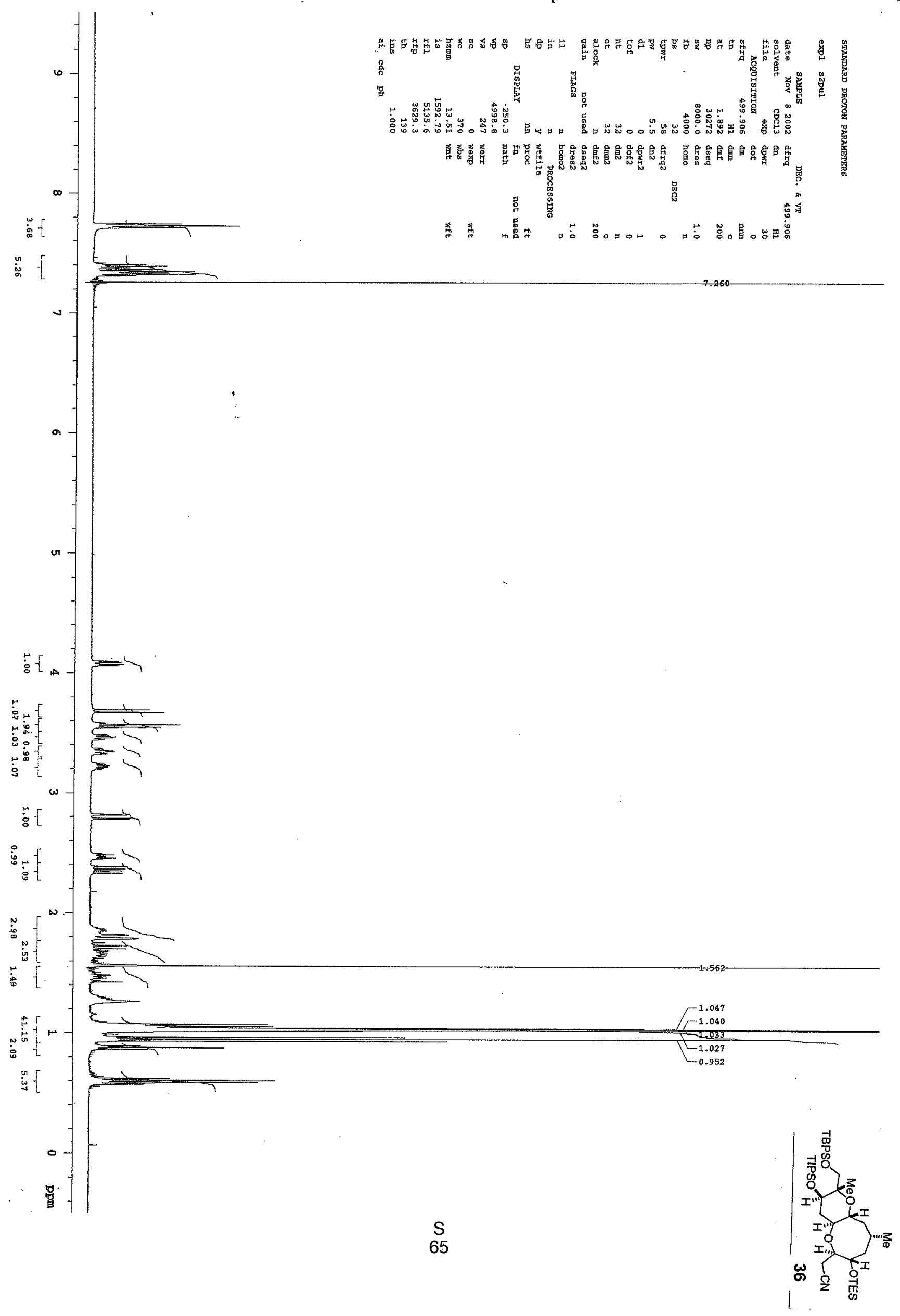




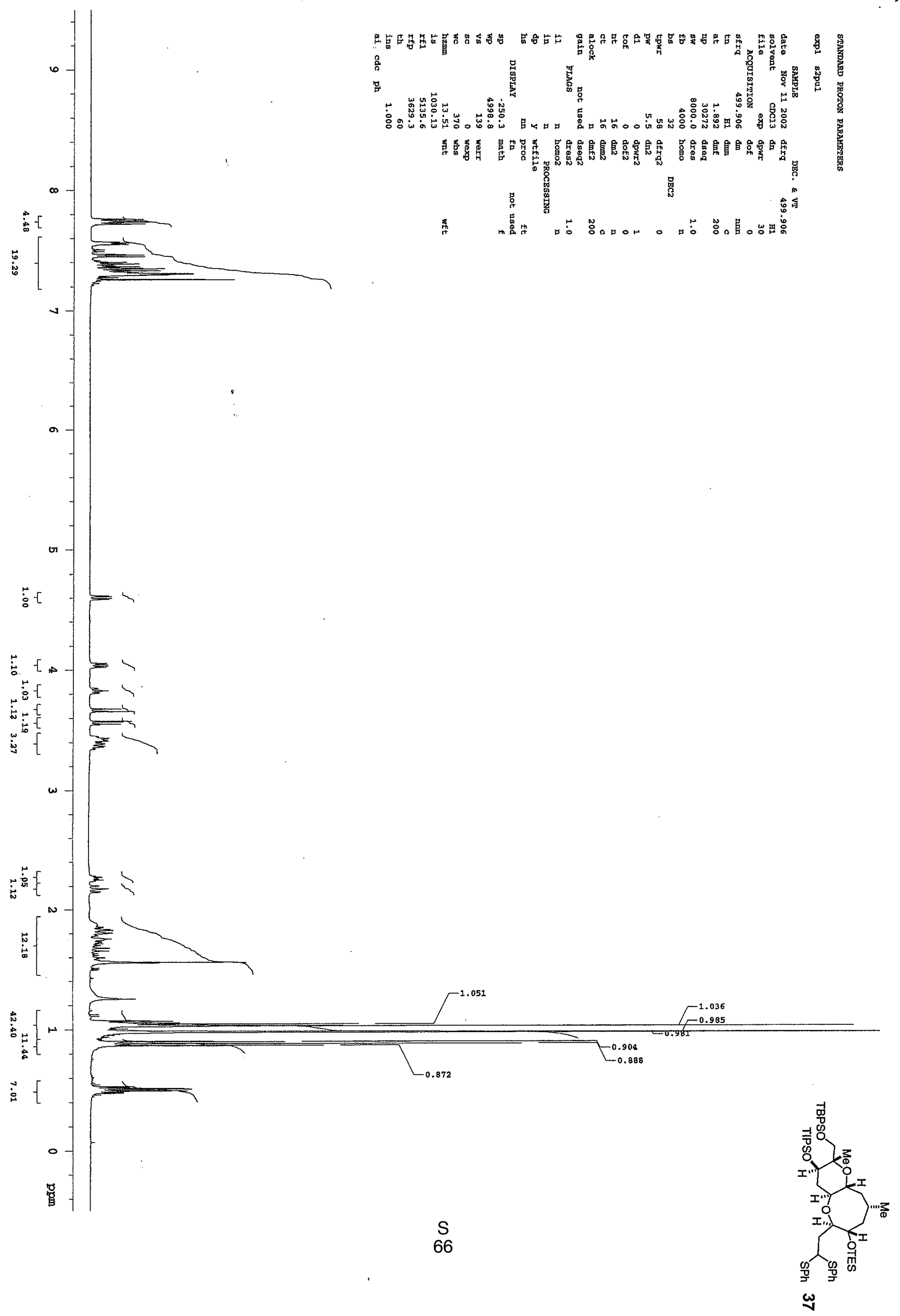




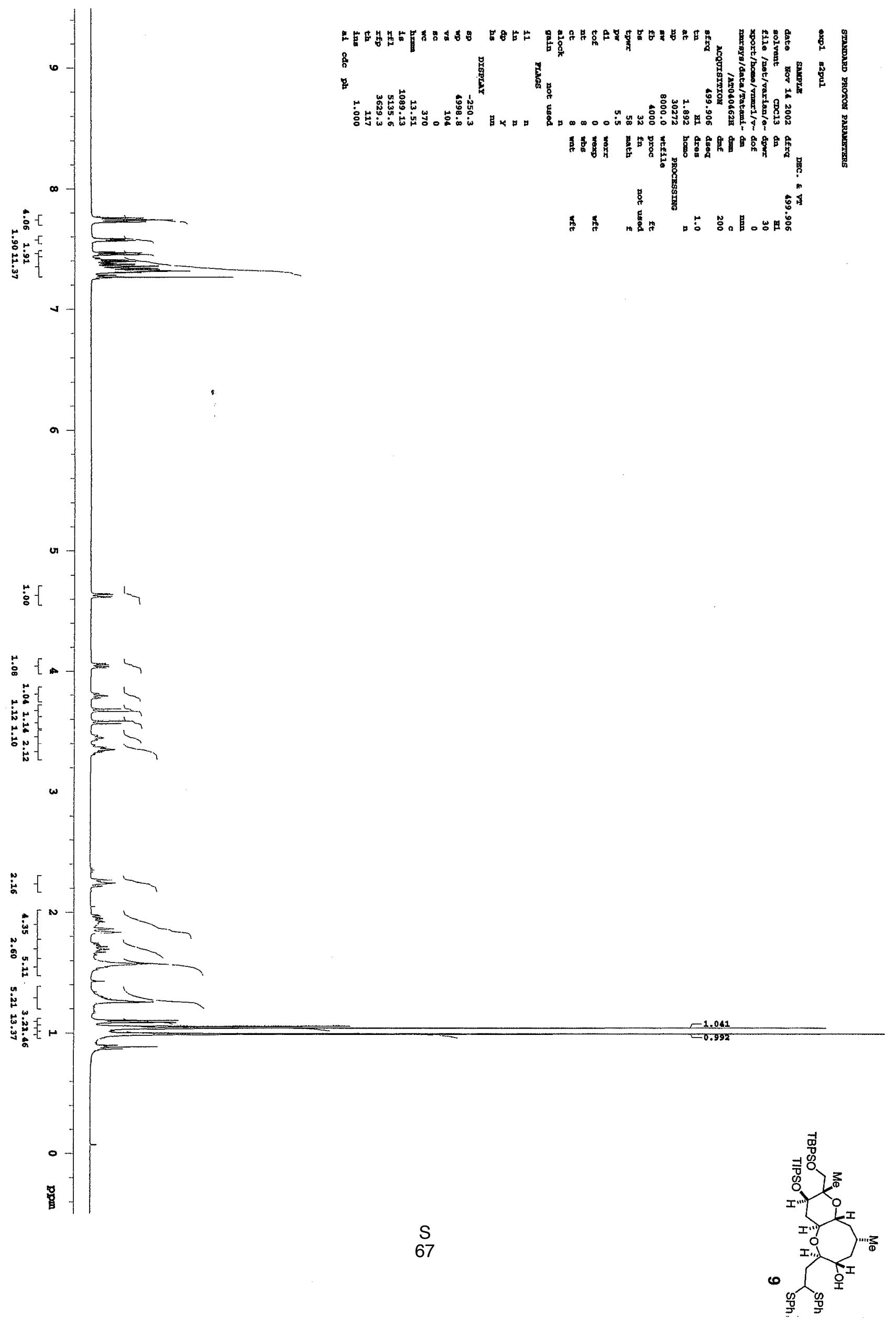




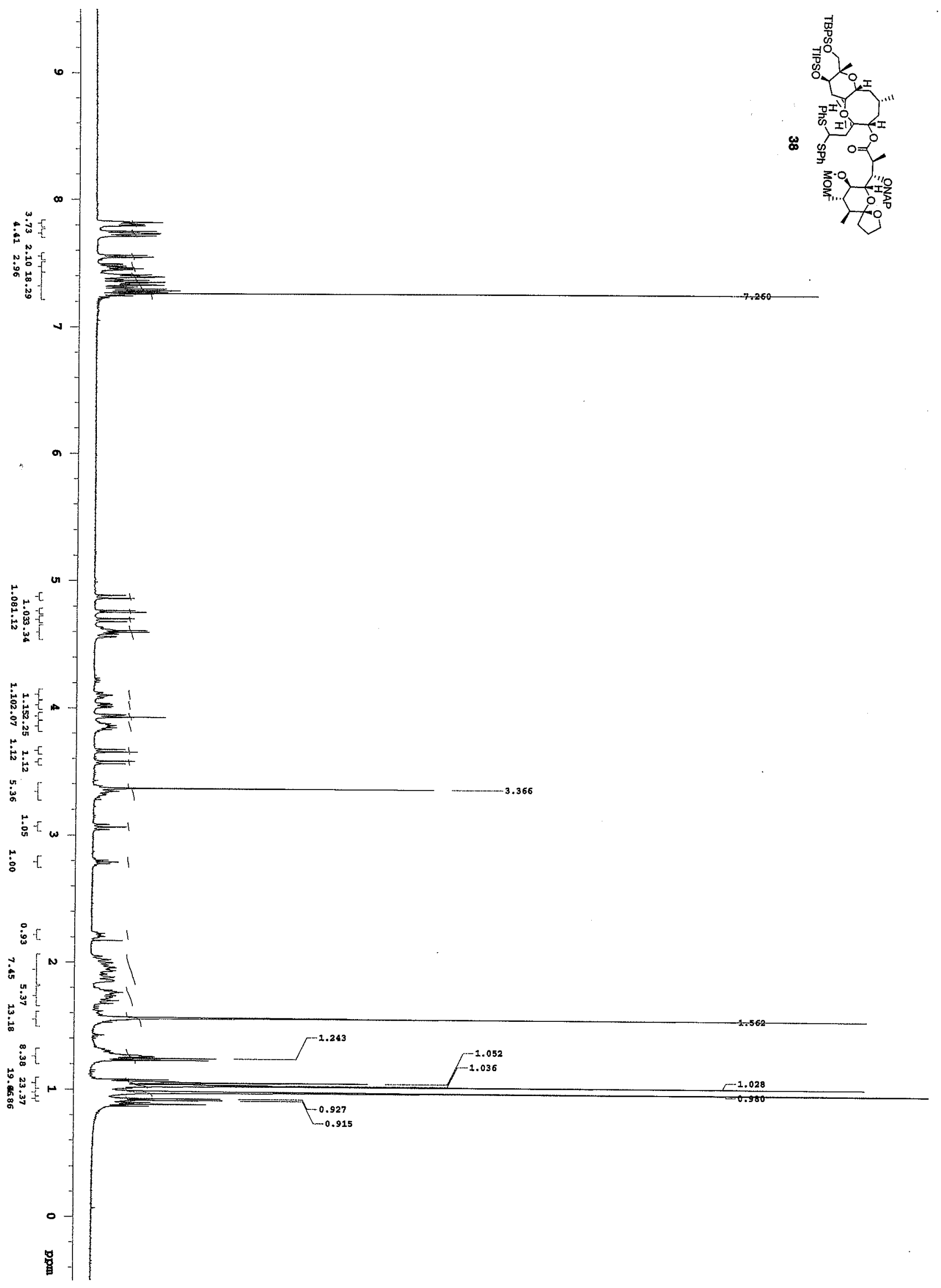




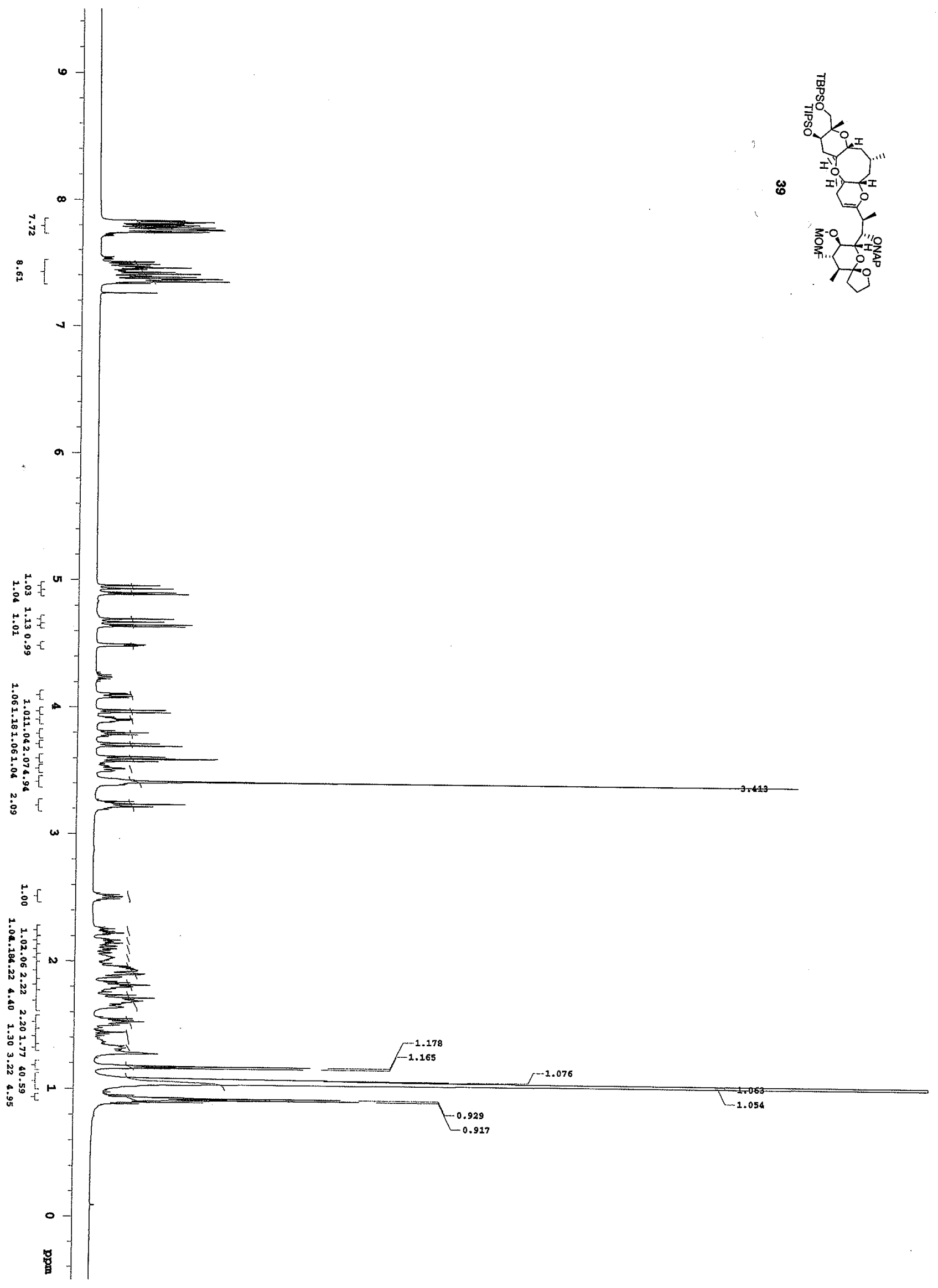




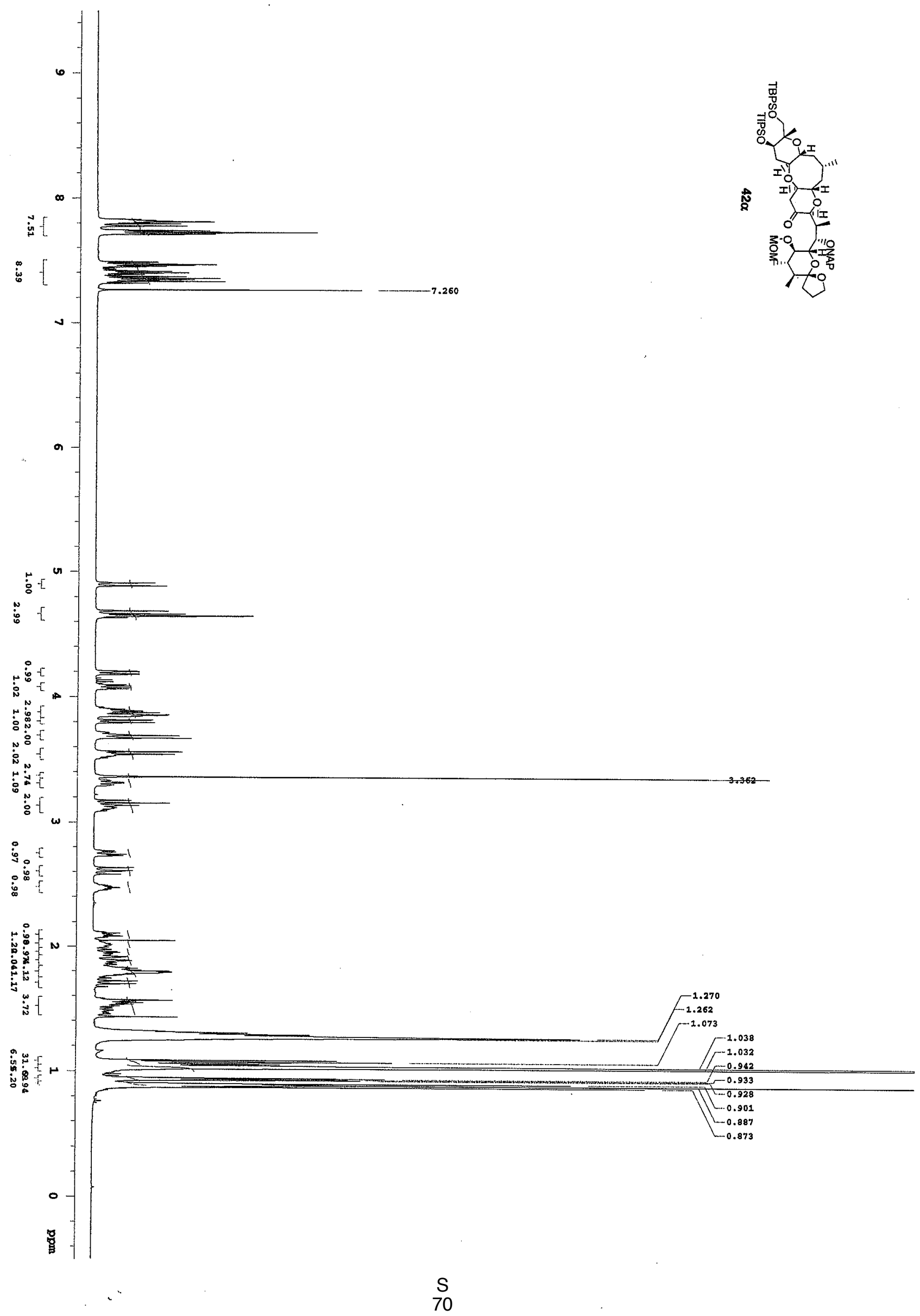




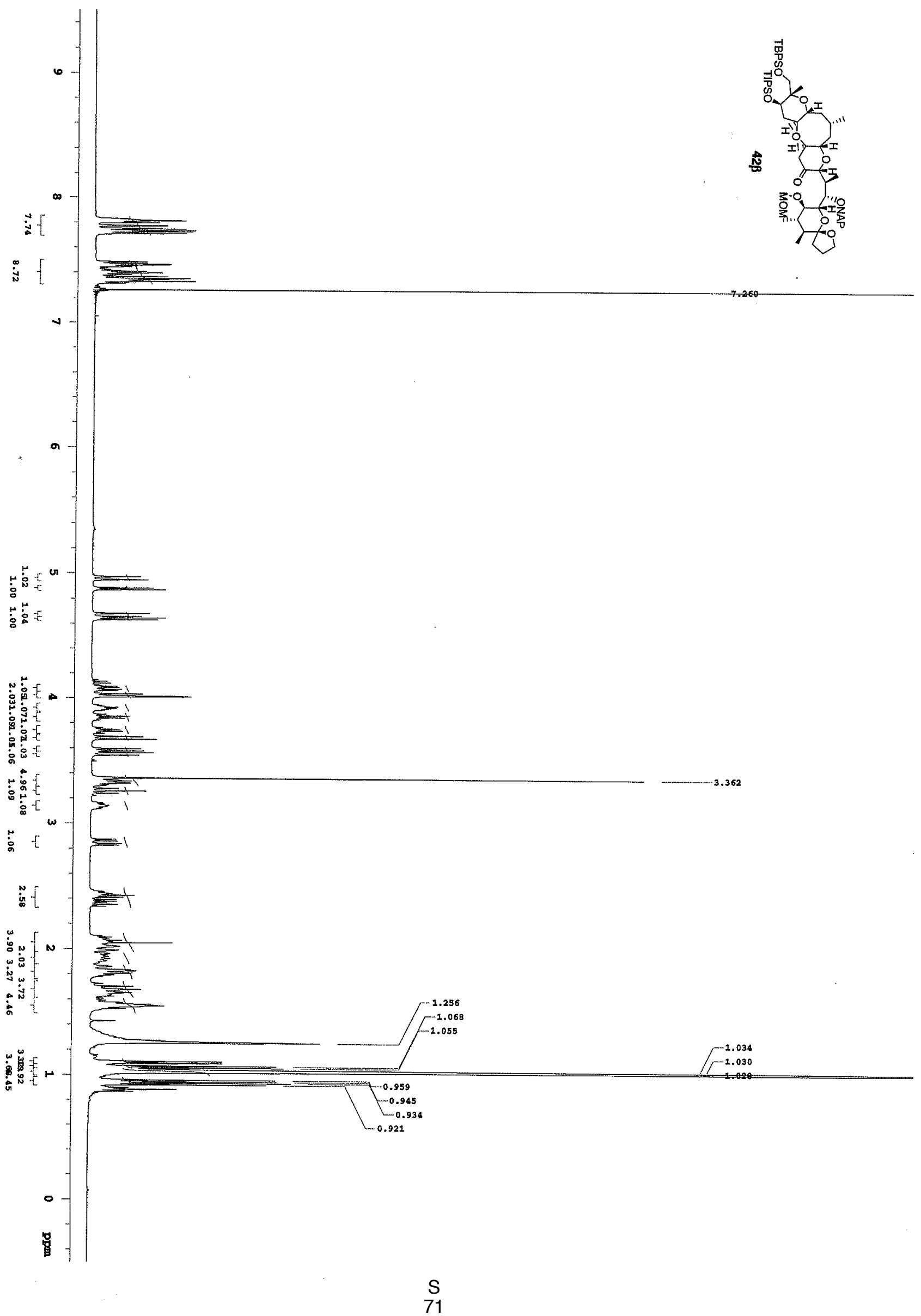




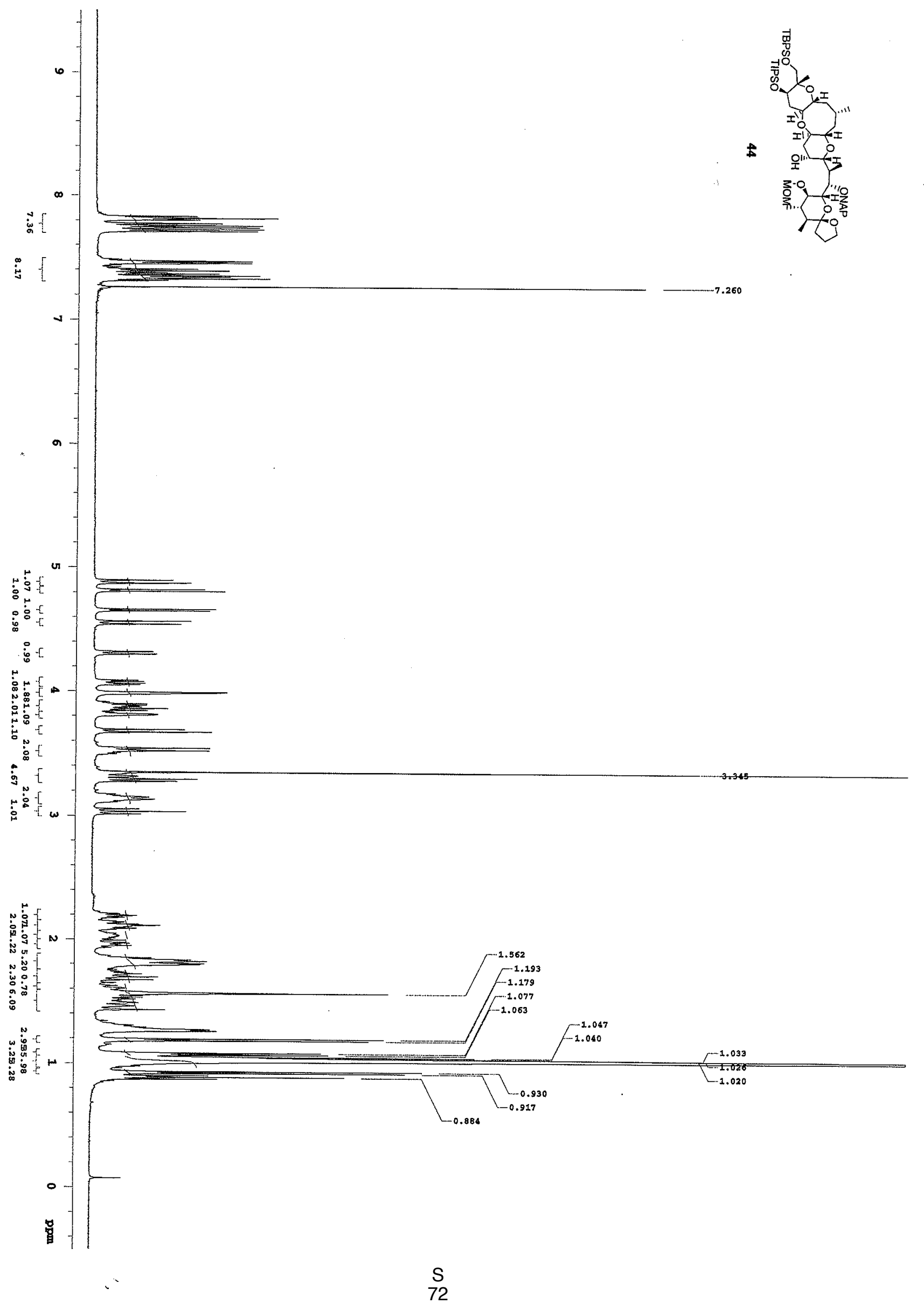




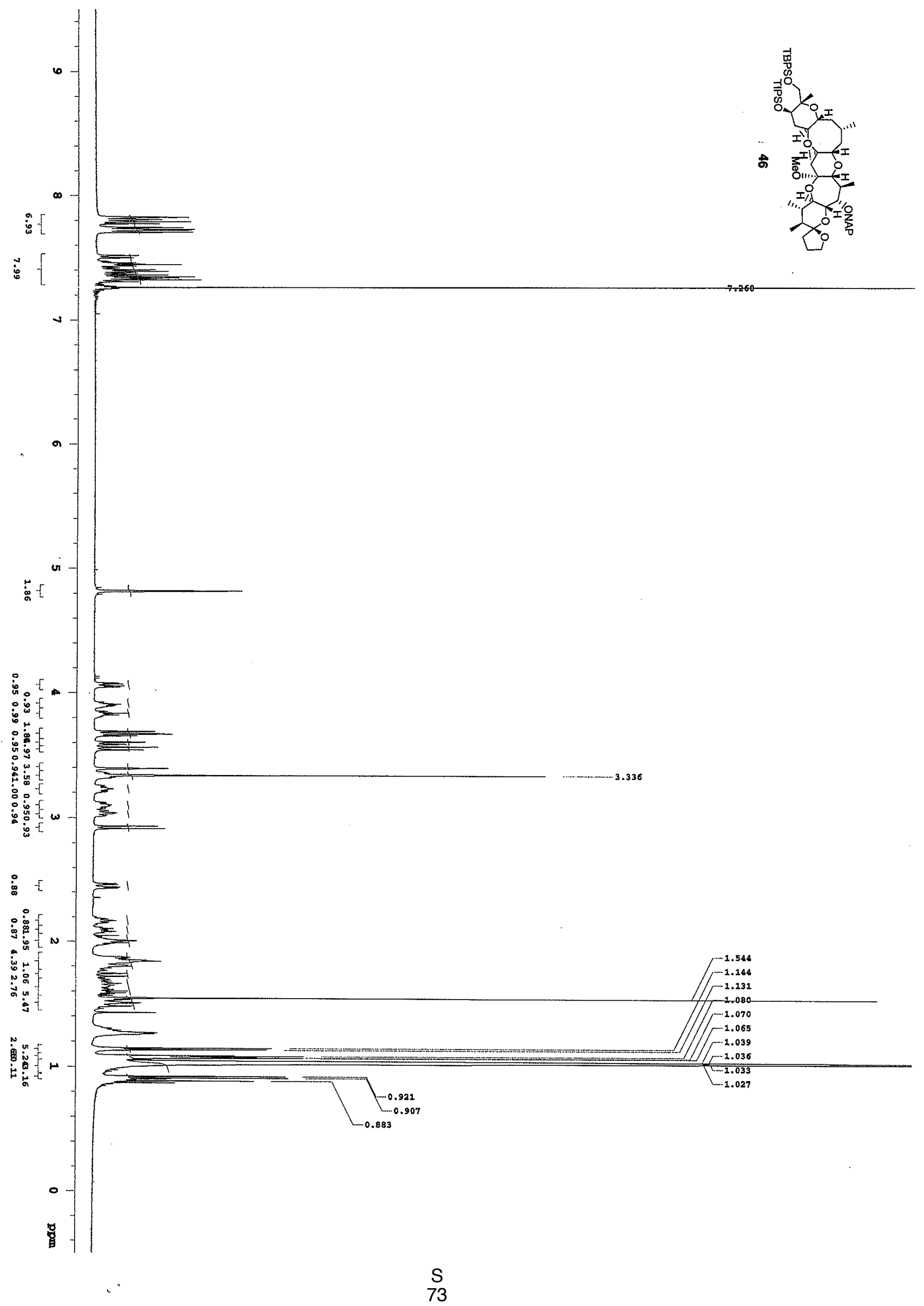




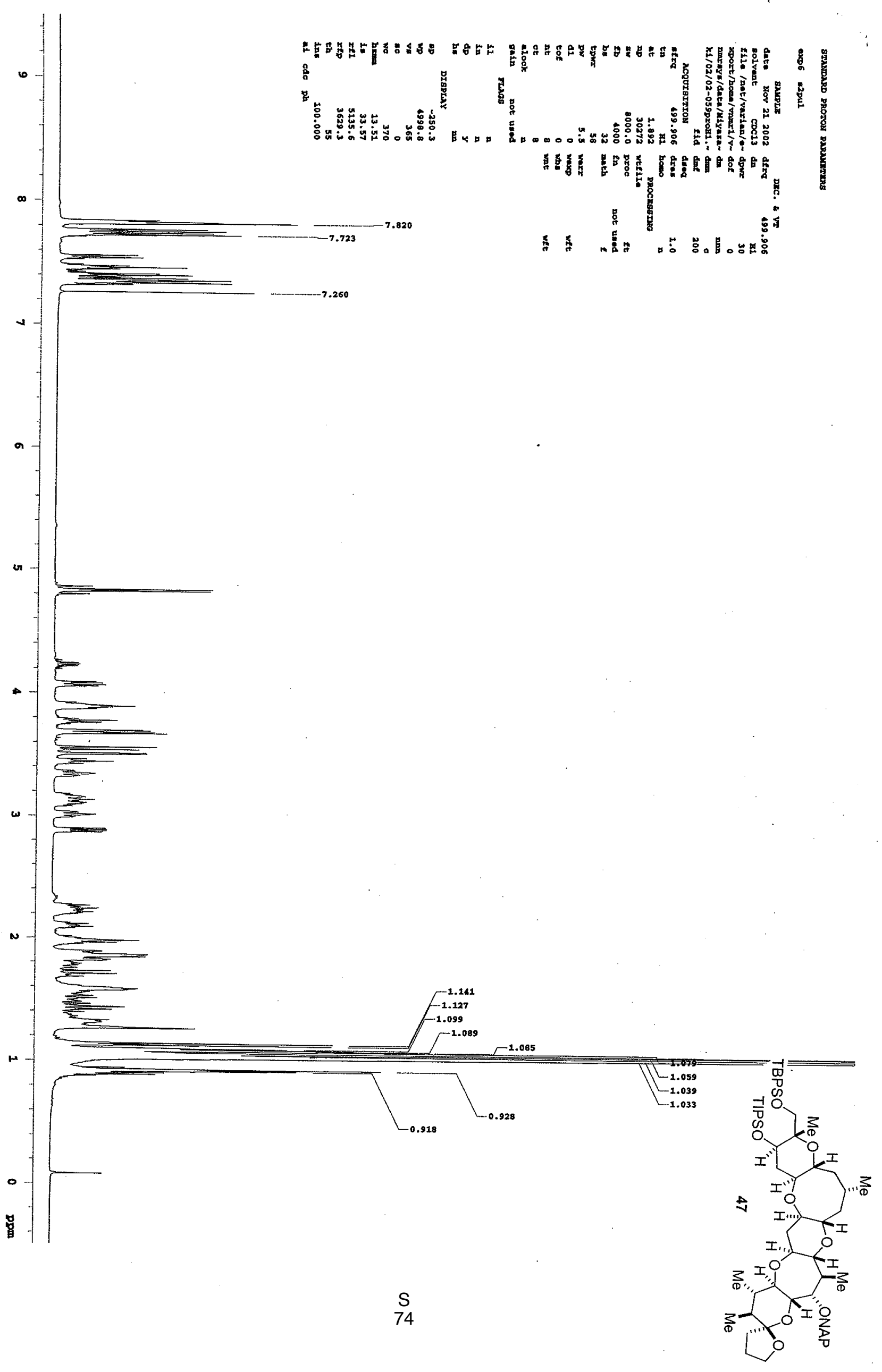




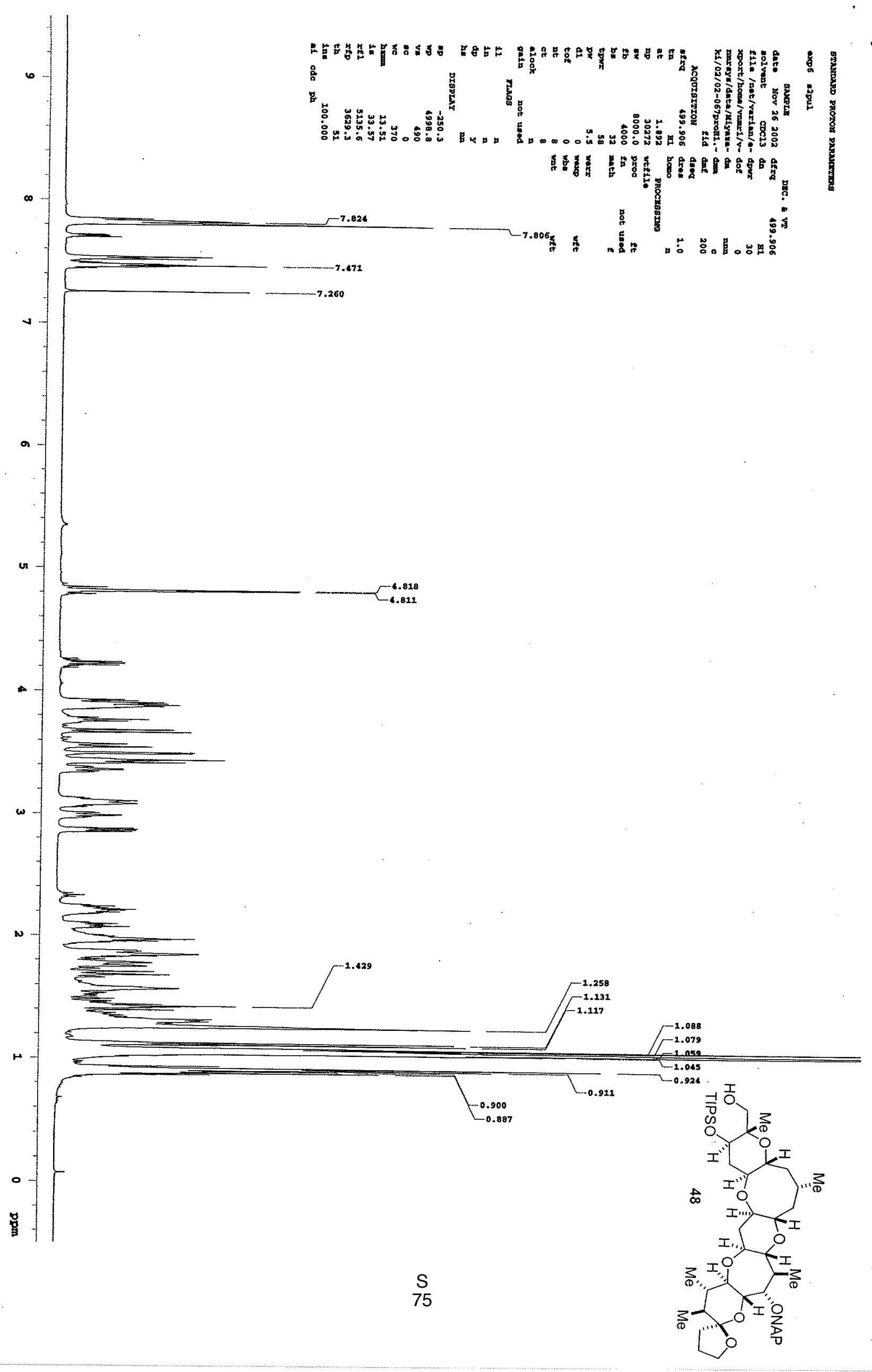




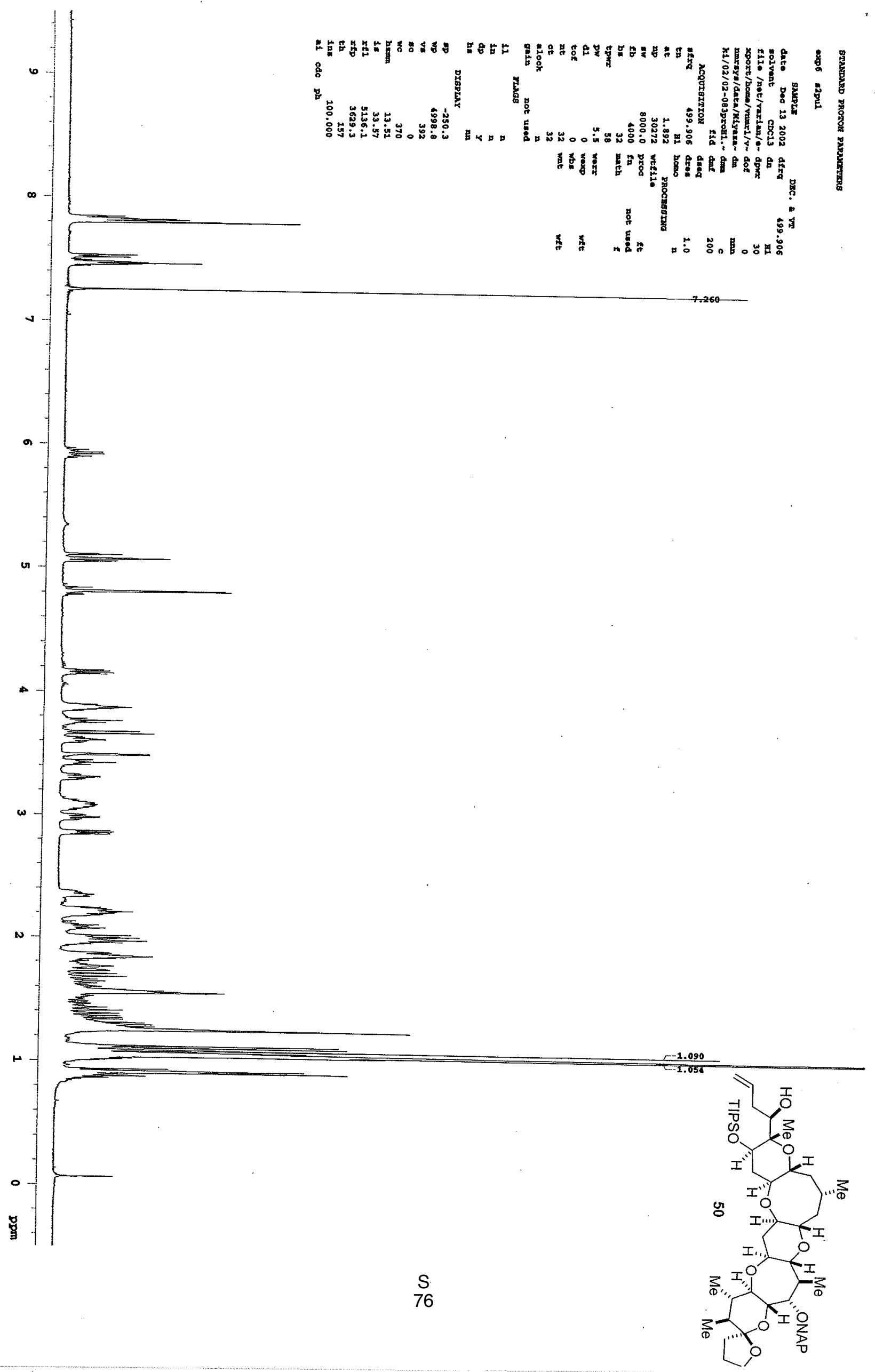




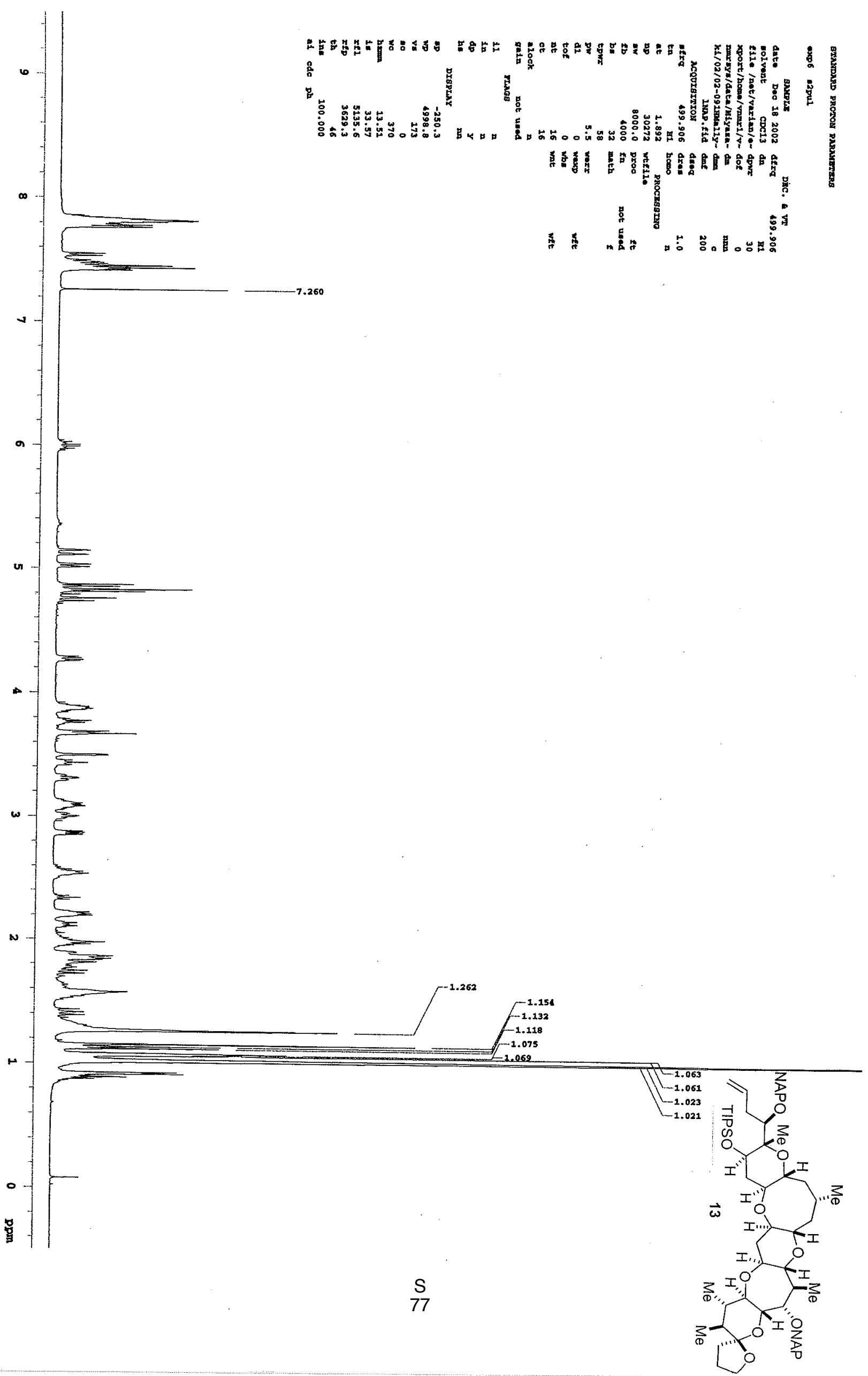




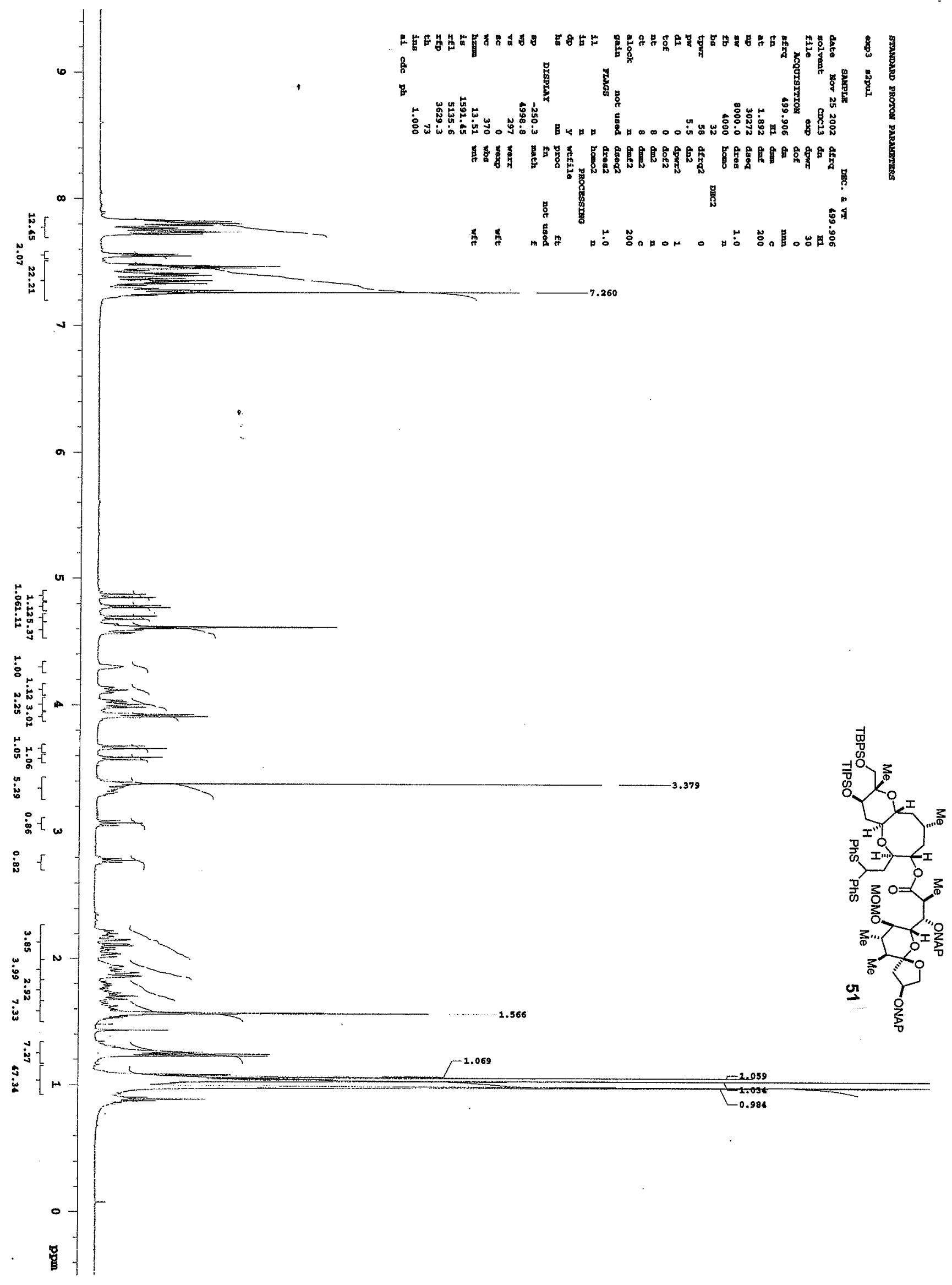




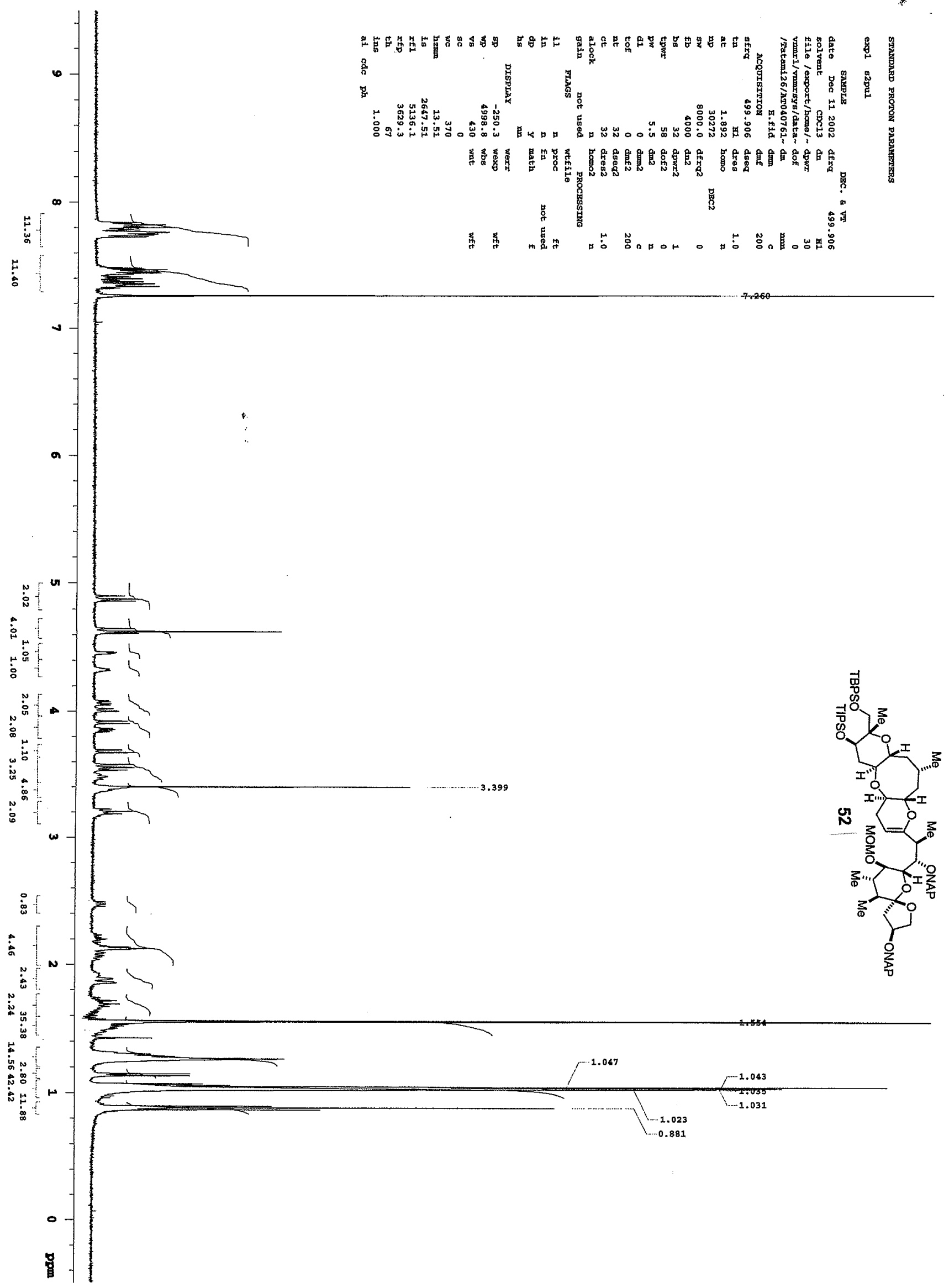




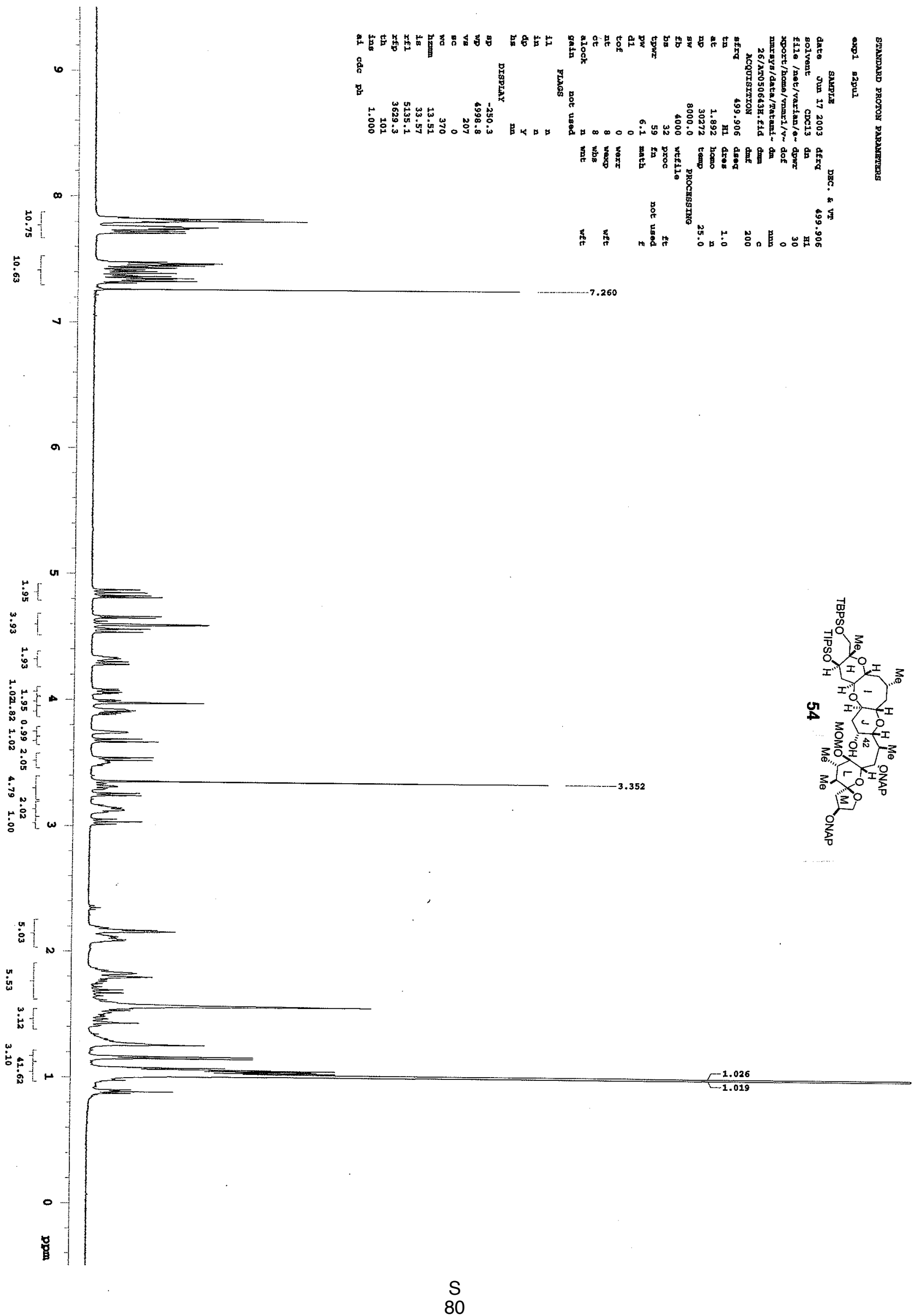




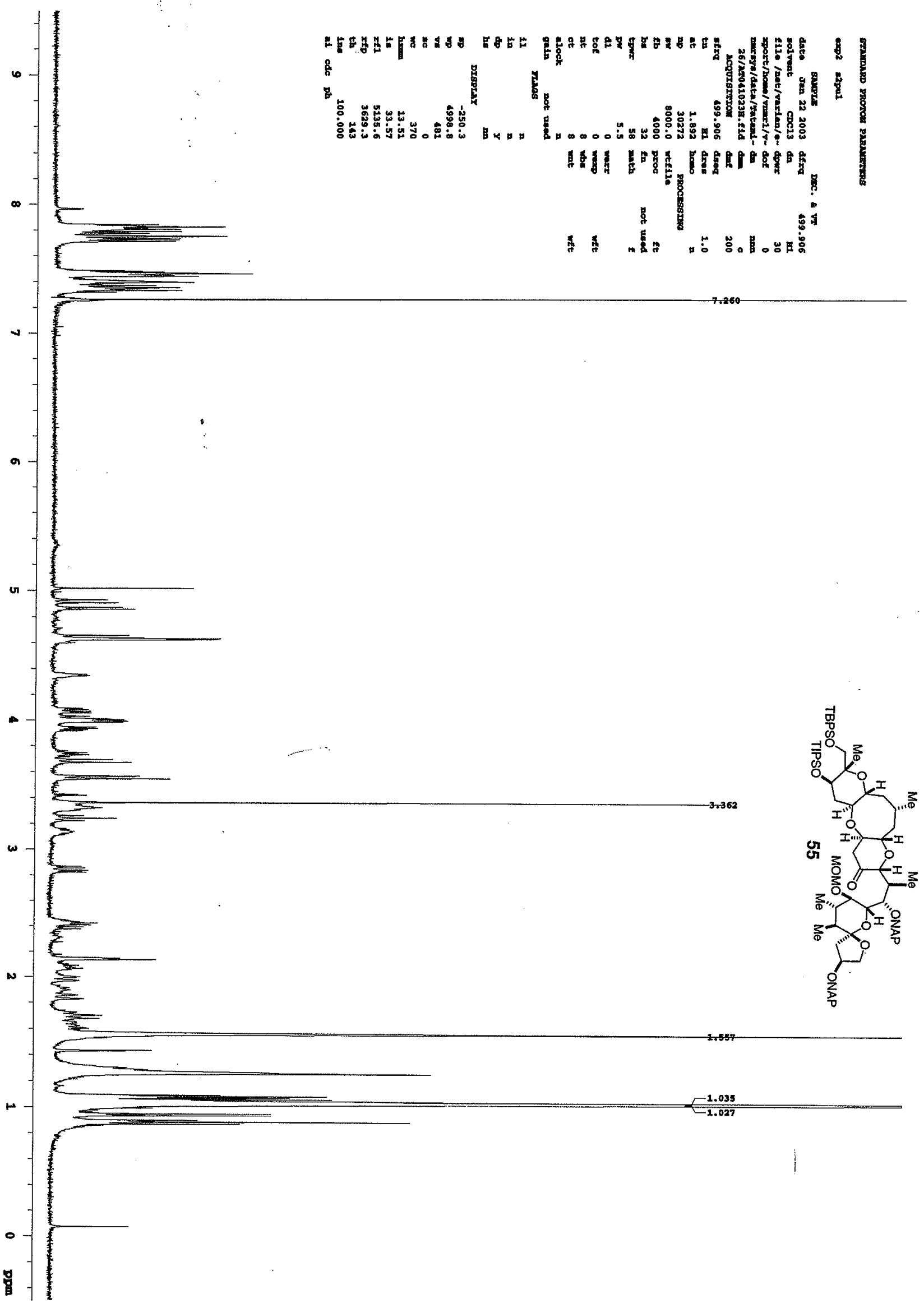




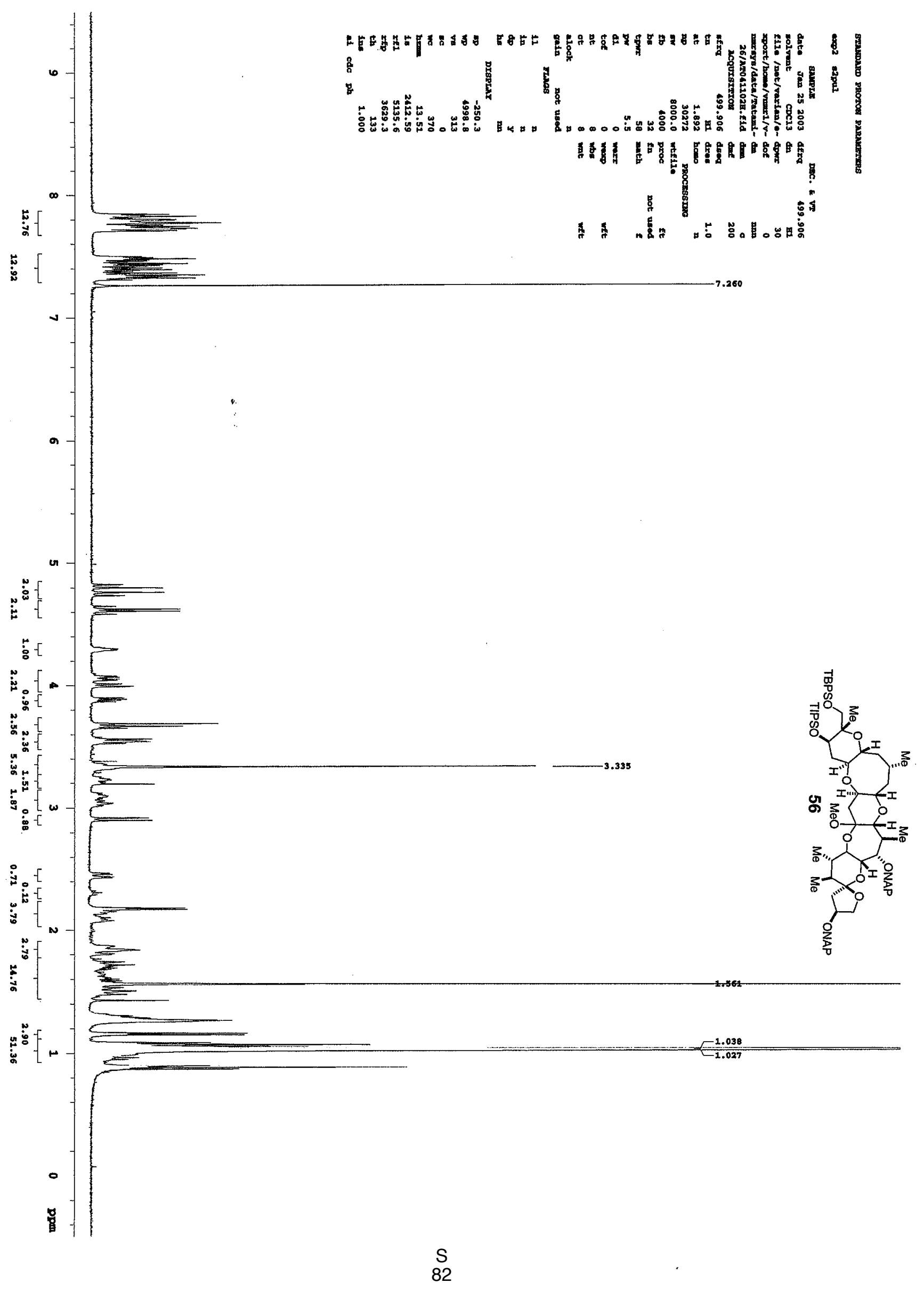




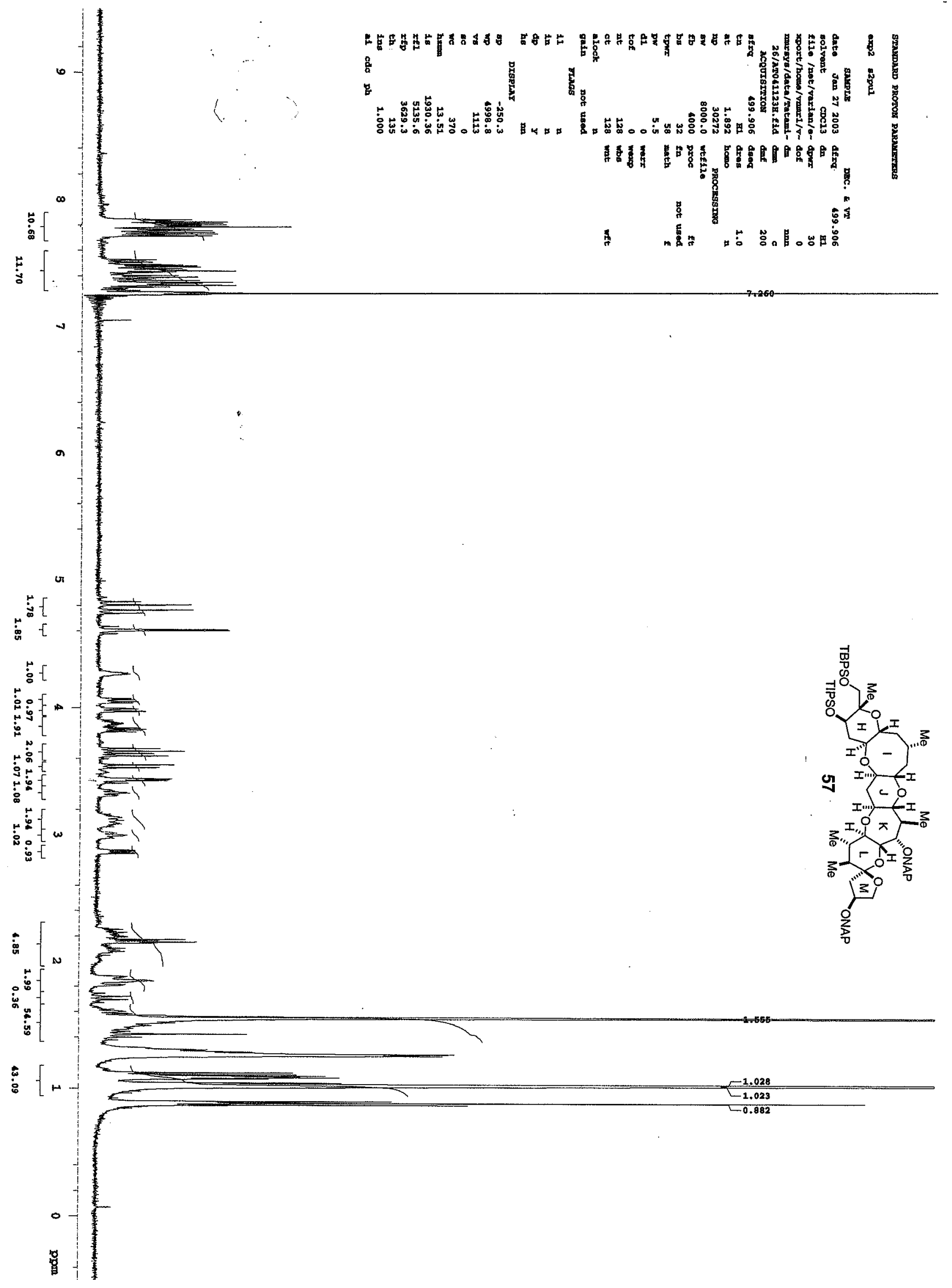




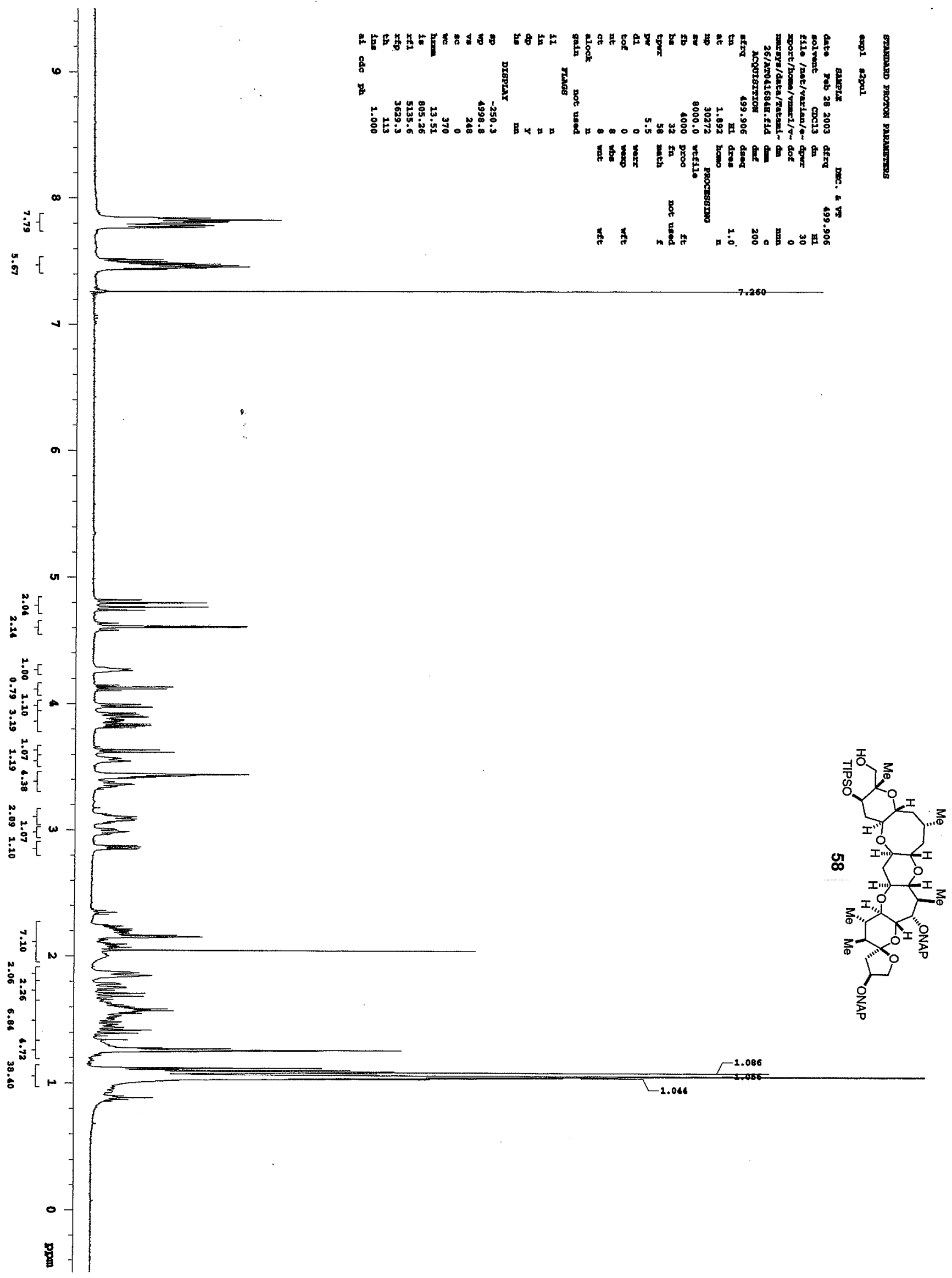




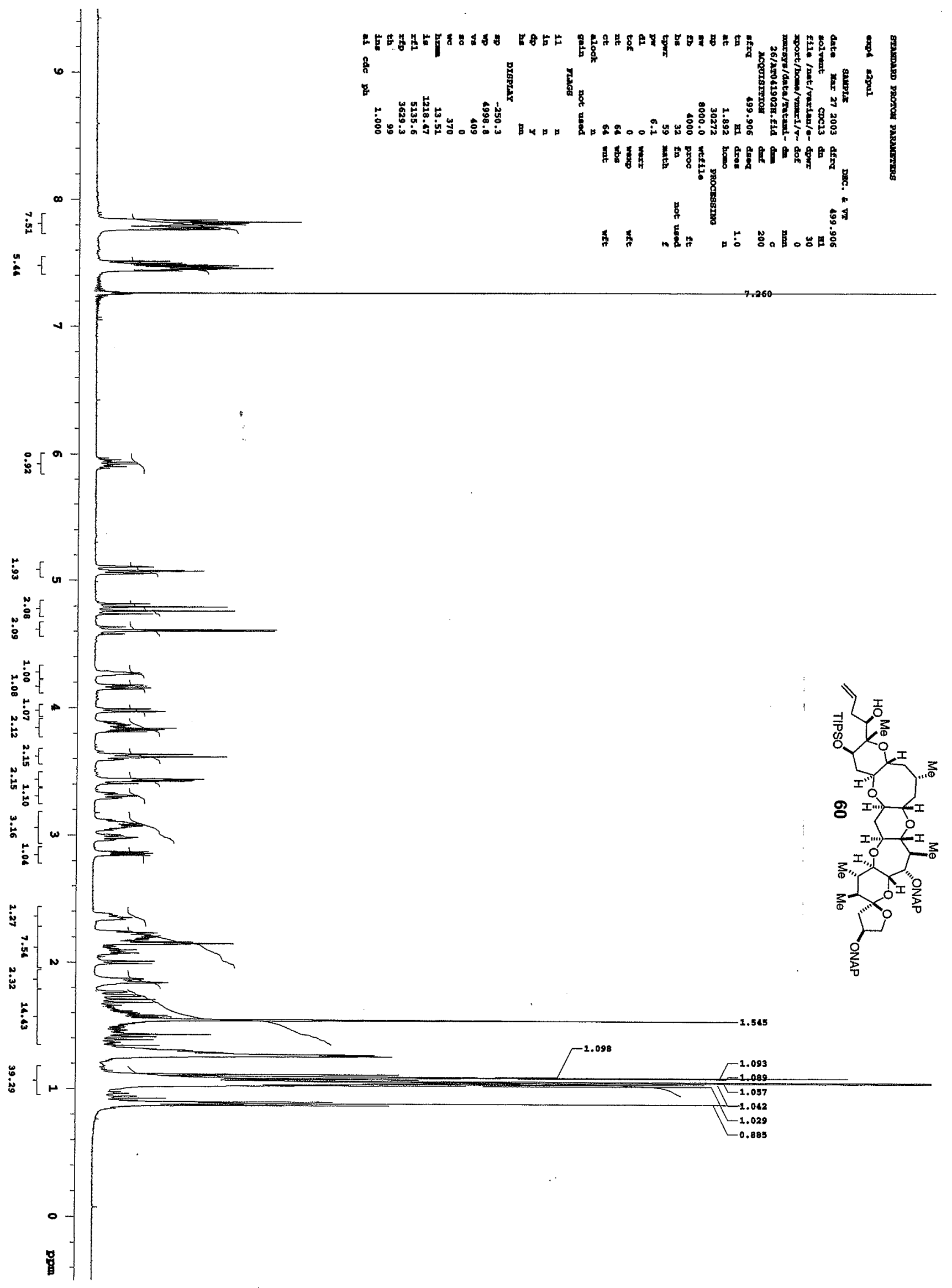




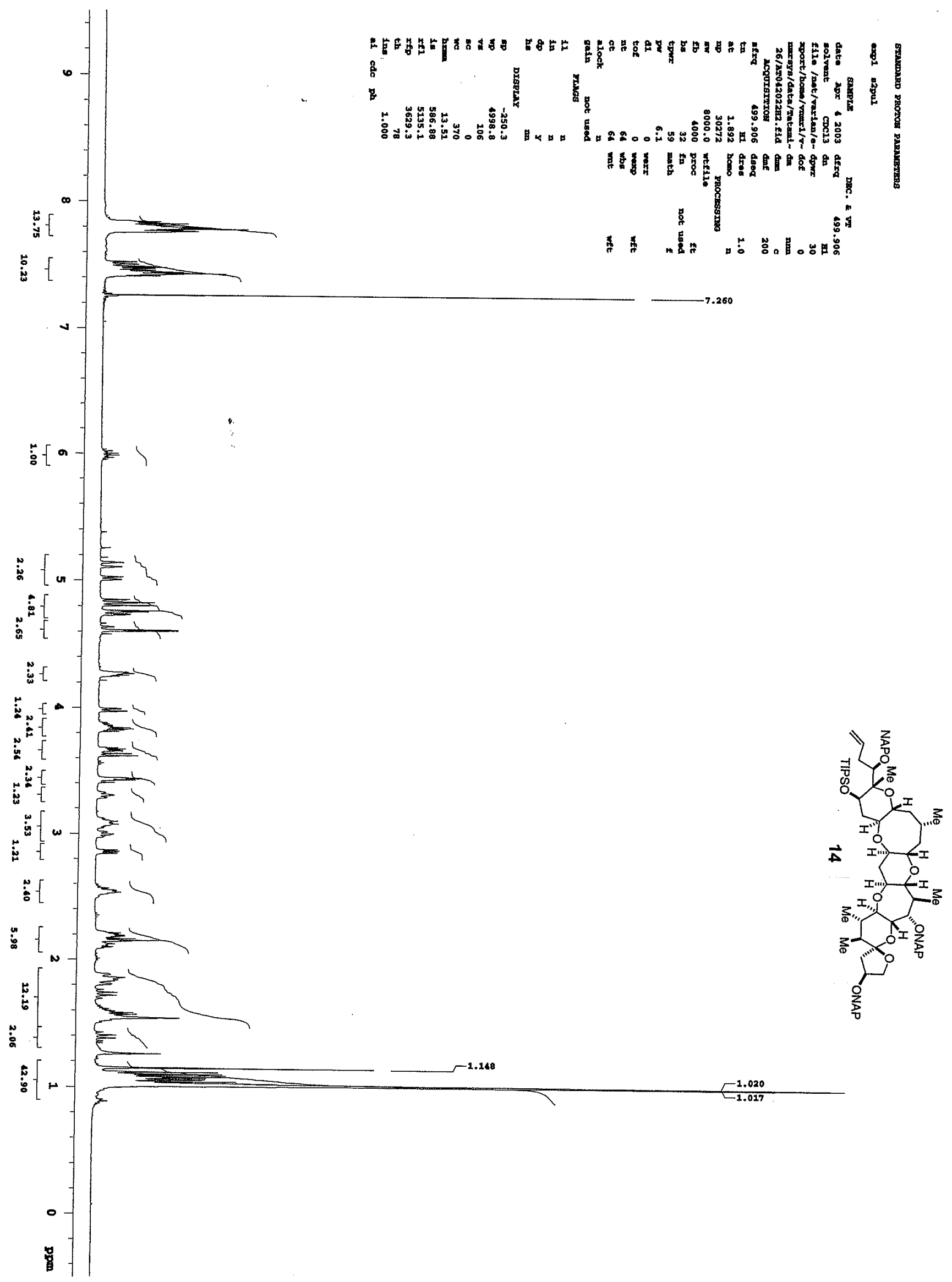

